\title{
Stellar variability in the MOA database \\ by
}

Simon Oliver Fletcher Wilkinson

A thesis submitted to Victoria University of Wellington in fulfilment of the requirements for the degree of Master of Science in Physics.

Victoria University of Wellington April 2003 


\section{Contents}

Abstract $\quad$ ix

Preface $\quad x$

Acknowledgements xii

1 Introduction $\quad 1$

1.1 MOA (Microlensing Observations in Astrophysics) . . . . . . . . . . . 1

1.1.1 Mount John University Observatory . . . . . . . . . . . . . . . 1

1.1.2 The MOA Boller \& Chivens telescope . . . . . . . . . . . . 2

1.1 .3 MOA-cam $1 \ldots \ldots \ldots . \ldots \ldots$

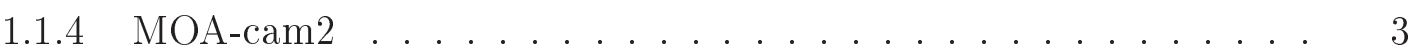

1.1 .5 MOA filters . . . . . . . . . . . . . . . 5

1.1.6 Observation fields . . . . . . . . . . . . . . . 5

1.1.7 Calibration ...................... 7

1.1 .8 Data storage . . . . . . . . . . . . . . . 7

2 The MOA CCD data reduction pipeline 9

2.1 Astronomical measurement . . . . . . . . . . . . . . . . . . . . 9

2.1.1 Brightness . . . . . . . . . . . . . . . . . . 9

2.1 .2 Time ............................. 10

2.2 Data reduction . . . . . . . . . . . . . . . . . 10

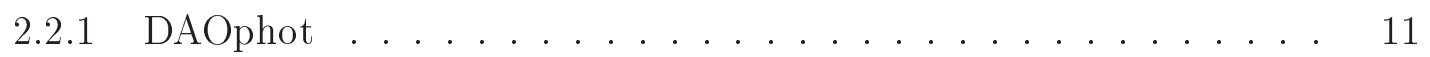

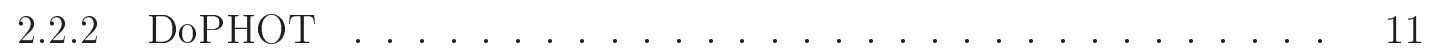

2.2 .3 Difference imaging . . . . . . . . . . . . . . . . . . 12

2.2.4 The Victoria University Wellington data reduction pipeline . . . . 12

2.3 Analysis tools for reduced data . . . . . . . . . . . . . . . . . 13

3 Miscellaneous variable stars $\quad 15$

3.1 Analysis tools used in searching for variable stars . . . . . . . . . . 15

3.1 .1 Variability index . . . . . . . . . . . . . . . . 15 
3.1 .2 Colour magnitude diagrams . . . . . . . . . . . . . . . 17

3.1.3 Period folding for periodic variables . . . . . . . . . . . . . . 20

3.2 Cepheid variables . . . . . . . . . . . . . . . . . . . . . . 21

3.2.1 The search for Cepheid variables . . . . . . . . . . . 23

3.2 .2 Lightcurves . . . . . . . . . . . . . . . . . . . . . 26

3.3 Long period variables . . . . . . . . . . . . . . . . . . . . . . . 28

3.3.1 The search for long period variables . . . . . . . . . . . . . 28

3.3 .2 Lightcurves ....................... 31

3.4 Eclipsing binaries . . . . . . . . . . . . . . . . . . 33

3.4.1 The search for eclipsing binaries . . . . . . . . . . . . . . 33

3.4 .2 Lightcurves . . . . . . . . . . . . . . . . . . 36

4 Gravitational microlensing $\quad 40$

4.1 History of gravitational lensing . . . . . . . . . . . . . . . . . . . 40

4.2 Gravitational microlensing surveys . . . . . . . . . . . . . . . . 41

4.3 Applications of microlensing surveys . . . . . . . . . . . . . . 43

4.3.1 Dark matter . . . . . . . . . . . . . . . . . 43

4.3.2 Planetary search . . . . . . . . . . . . . . . . 44

4.4 Physics of microlensing . . . . . . . . . . . . . . . . 45

4.5 Modelling for microlensing curves . . . . . . . . . . . . . . . . . 48

4.6 Microlensing events . . . . . . . . . . . . . . . . . . . . . . 52

4.6 .1 The 1997 LMC data . . . . . . . . . . . . . . . 54

4.6.2 The 2000 Galactic Bulge data . . . . . . . . . . . . . . 56

5 Planetary transits $\quad 57$

5.1 Extrasolar planets . . . . . . . . . . . . . . . 57

5.1 .1 Detection methods . . . . . . . . . . . . . . 57

5.1 .2 Planetary transits . . . . . . . . . . . . . . 58

5.2 Transit simulation and a phase space search for periodic transits . . . . . 62

5.3 The search for planetary transits . . . . . . . . . . . 68

5.3.1 Statistical analysis of the MOA data . . . . . . . . . . . 68

5.3.2 Planetary transit lightcurves . . . . . . . . . . . . . . . . . 70

5.3 .3 Candidate lightcurves . . . . . . . . . . . . . . . . 75

5.3.4 Further analysis of planetary transit transit5829. . . . . . . . . 81

6 Conclusions 
$\begin{array}{ll}\text { A File examples } & 87\end{array}$

A.1 Data reduction pipeline photometry files . . . . . . . . . . . 87

A.1.1 Example of the pipeline .info file . . . . . . . . . . . . 87

A.2 Analysis files . . . . . . . . . . . . . . . . . . . . 89

A.2.1 Example of the colour-magnitude data file . . . . . . . . . . 89

A.2.2 Example of a folded lightcurve file . . . . . . . . . . . . . . . . 90

$\begin{array}{ll}\text { B Program structure } & 91\end{array}$

$\begin{array}{lr}\text { C Catalogue } & 96\end{array}$

C.1 Catalogue of MOA LMC Cepheid variables . . . . . . . . . . . . . . . 98

C.2 Catalogue of MOA LMC eclipsing binaries . . . . . . . . . . . . . . . 101

C.3 Catalogue of MOA Galactic Bulge eclipsing binaries . . . . . . . . . . . . 103

C.4 Catalogue of MOA Galactic Bulge potential planetary transits . . . . . . 104 


\section{List of Figures}

1.1 Seeing conditions at Mount John University Observatory . . . . . . . . 2

1.2 MOA-cam1 CCD layout . . . . . . . . . . . . . . . . . 4

1.3 MOA-cam2 CCD layout . . . . . . . . . . . . . . . . 4

1.4 Response functions for the two MOA broadband filters . . . . . . . . . 5

1.5 The MOA ngb 1, ngb 2 and nlmc 1 fields . . . . . . . . . . . 6

1.6 The MOA NGC 3201 calibration field . . . . . . . . . . . . . . 6

3.1 Hertzsprung-Russell diagram . . . . . . . . . . . . . . . . . . 18

3.2 Colour magnitude diagram of NGC $3201 \ldots \ldots$. . . . . . . . . . 19

3.3 Colour magnitude diagram of MOA field nlmc $0-1 \ldots$. . . . . . . . . 20

3.4 Period luminosity relationship for the Cepheid variables found the MOA nlmc 1 star field . . . . . . . . . . . . . . . . . . . . 24

3.5 The MOA-red, MOA-blue and colour difference plots of the Cepheid variable cepheid9436. . . . . . . . . . . . . . . . . 25

3.6 Folded lightcurves of the MOA-red observations for the LMC Cepheid variables cepheid1623 and cepheid1731 . . . . . . . . . . . 26

3.7 Folded lightcurves of the MOA-red observations for the LMC Cepheid variables cepheid1828 and cepheid2308 . . . . . . . . . . . 26

3.8 Folded lightcurves of the MOA-red observations for the LMC Cepheid variables cepheid3082 and cepheid4604 . . . . . . . . . . . 26

3.9 Folded lightcurves of the MOA-red observations for the LMC Cepheid variables cepheid4991 and cepheid6142 . . . . . . . . . . . . 27

3.10 Folded lightcurves of the MOA-red observations for the LMC Cepheid variables cepheid8550 and cepheid9642 . . . . . . . . . . . . . 27

3.11 Folded lightcurves of the MOA-red observations for the LMC Cepheid variables cepheid10409 and cepheid10966 . . . . . . . . . . . .

3.12 The MOA-red, MOA-blue and Colour index plots of the RV Tauri variable long_period $4178 \ldots \ldots \ldots \ldots \ldots \ldots$

3.13 Lightcurves of the MOA-red observations for the LMC long period variables long_period2006 and long_period $3627 \ldots \ldots \ldots$. . . . . . . . . . . . 
3.14 Lightcurves of the MOA-red observations for the LMC long period variables long_period4411 and long_period6704 . . . . . . . . . . . . .

3.15 Lightcurves of the MOA-red observations for the LMC long period variables long_period10389 and long_period10646 . . . . . . . . . . . .

3.16 Lightcurves of the MOA-red observations for the LMC long period variables long_period10692 and long_period10787 . . . . . . . . . . . .

3.17 Lightcurves of the MOA-red observations for the LMC long period variables long_period10912 and long_period11343 . . . . . . . . . . . . .

3.18 Lightcurves of the MOA-red observations for the LMC long period variables long_period19522 and long_period24952 . . . . . . . . . . . . .

3.19 The MOA-red, MOA-blue and colour index plots of the eclipsing binary

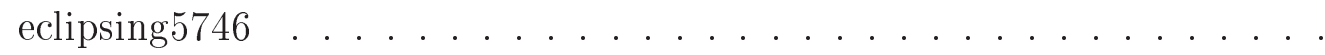

3.20 Folded lightcurves of the MOA-red observations for the LMC eclipsing binaries eclipsing1736 and eclipsing2138 . . . . . . . . . . . . .

3.21 Folded lightcurves of the MOA-red observations for the LMC eclipsing binaries eclipsing2301 and eclipsing2654 . . . . . . . . . . . . .

3.22 Folded lightcurves of the MOA-red observations for the LMC eclipsing binaries eclipsing2819 and eclipsing4811 . . . . . . . . . . . . .

3.23 Folded lightcurves of the of the MOA-red observations for the LMC eclipsing binaries eclipsing5078 and eclipsing5462 . . . . . . . . . . .

3.24 Folded lightcurves of the of the MOA-red observations for the LMC eclipsing binaries eclipsing 8138 and eclipsing $9411 \ldots \ldots$. . . . . . . . .

3.25 Folded lightcurves of the of the MOA-red observations for the LMC eclipsing binaries eclipsing10089 and eclipsing11441 . . . . . . . . . .

3.26 Folded lightcurves of the of the MOA-red observations for the Galactic Bulge eclipsing binaries eclipsing486_a and eclipsing784_a . . . . . . . . .

3.27 Folded lightcurves of the of the MOA-red observations for the Galactic Bulge eclipsing binaries eclipsing1010_a and eclipsing2143_a . . . . . . . .

3.28 Folded lightcurves of the of the MOA-red observations for the Galactic Bulge eclipsing binaries eclipsing2476_a and eclipsing5174_a . . . . . . . .

3.29 Folded lightcurves of the of the MOA-red observations for the Galactic Bulge eclipsing binaries eclipsing5286_a and eclipsing5441_a . . . . . . . .

3.30 Folded lightcurves of the of the MOA-red observations for the Galactic Bulge eclipsing binaries eclipsing13327_a and eclipsing16391_a . . . . . .

3.31 Folded lightcurves of the of the MOA-red observations for the Galactic Bulge eclipsing binaries eclipsing25968_a and eclipsing26988_a . . . . . . 
4.1 The geometry of the separation between the lens object and the source objects in the plane of the lensing object. . . . . . . . . . . . 46

4.2 Comparison of the microlensing, Gaussian and Lorentzian curves . . . . . 47

4.3 The increasing accuracy of the microlensing modelling program achieved by increasing the number of passes of the program . . . . . . . . . . 50

4.4 An incorrectly modelled lightcurve . . . . . . . . . . . . . . 51

4.5 Lightcurve of the potential microlensing event microcurve1309 . . . . . . 54

4.6 Lightcurve of the potential microlensing event microcurve2173 . . . . . 54

4.7 Lightcurve of the potential microlensing event microcurve4333 . . . . . 54

4.8 Lightcurve of the potentia'l microlensing event microcurve6344 . . . . . . 55

4.9 Lightcurve of the potential microlensing event microcurve10751 . . . . 55

4.10 Lightcurve of the potential microlensing event microcurve12674 . . . . 55

4.11 Lightcurve of the microlensing event ngb1-2-2717 . . . . . . . . . 56

4.12 Lightcurve of the microlensing event ngb11-2-1142 . . . . . . . . 56

5.1 Planetary transit models . . . . . . . . . . . . . . . . . . . 60

5.2 Planetary transit HD $209458 \ldots \ldots \ldots$. . . . . . . . . . . 61

5.3 Simulated lightcurve of a planetary transit . . . . . . . . . . . . . 63

5.4 Observation points folded into the first trial period . . . . . . . . . 64

5.5 Normal distribution of cross correlation values for a simulated transit . . 65

5.6 Folded lightcurve of a simulated transit . . . . . . . . . . . . 66

5.7 A sixth order polynomial compared to the planetary transit models . . . 67

5.8 Histograms of the standard deviation for the MOA fields nlme $0-1$ and ngb $2-1 \ldots \ldots \ldots \ldots$

5.9 Histograms of the time difference between successive observations for the MOA field ngb 2-2. . . . . . . . . . . . . . . . . 69

5.10 Photometry of MOA fields ngb $1-1$ and ngb $2-1 \ldots . . \ldots 72$

5.11 Normal distribution of cross correlation values for the potential transit curve transit3156 . . . . . . . . . . . . . . . . . . 73

5.12 Folded lightcurves of the'very interesting' transit event transit5829 . . . . 75

5.13 The finding chart for the 'very interesting' transit event transit5829 . . . 75

5.14 Folded lightcurves of the potential transit event transit228 . . . . . . 76

5.15 Folded lightcurves of the potential transit event transit335 . . . . . . . 76

5.16 Folded lightcurves of the potential transit event transit1337 . . . . . . . 76

5.17 Folded lightcurves of the potential transit event transit2722 . . . . . . . . 77

5.18 Folded lightcurves of the potential transit event transit2859 . . . . . . . . 77

5.19 Folded lightcurves of the potential transit event transit2949 . . . . . . . . 77

5.20 Folded lightcurves of the potential transit event transit3156 . . . . . . . 78 
5.21 Folded lightcurves of the potential transit event transit3175 . . . . . . 78

5.22 Folded lightcurves of the potential transit event transit3816 . . . . . . 78

5.23 Folded lightcurves of the potential transit event transit4567 . . . . . . . 79

5.24 Folded lightcurves of the potential transit event transit4741 . . . . . . . 79

5.25 Folded lightcurves of the potential transit event transit5479 . . . . . . . . 79

5.26 Folded lightcurves of the potential transit event transit5610 . . . . . . . 80

5.27 Folded lightcurves of the potential transit event transit12414 . . . . . . . 80

5.28 Folded lightcurves of the potential transit event transit18269 . . . . . . . 80

5.29 Folded lightcurves of the potential transit event transit5829, containing both the 2000 and 2001 observations . . . . . . . . . . . . . . 81

5.30 Folded lightcurves of the potential transit event transit5829, containing only 2001 observations. . . . . . . . . . . . . . . . . . 81

6.1 Transit event OGLE-TR-40, discovered by the OGLE microlensing group 85

B.1 The structure of the search.cpp database extraction programs. . . . . . 95

C.1 The location of the variable stars found in the MOA nlmc 1 field overlaid with the position of the field boundaries. . . . . . . . . . . . . . . 97 


\section{List of Tables}

4.1 Modelling of simulated microlensing data . . . . . . . . . . . . . 49

5.1 Statistics of the 2000 Galactic Bulge fields investigated for planetary transits 71

5.2 Categories for potential transit lightcurves . . . . . . . . . . . 74

C.1 The properties of the LMC Cepheid variables . . . . . . . . . . . 100

C.2 The properties of the LMC eclipsing binaries . . . . . . . . . . . . . 102

C.3 The properties of the Galactic Bulge eclipsing binaries . . . . . . . . . 103

C.4 The properties of the Galactic Bulge interesting and very interesting potential planetary transits . . . . . . . . . . . . . . . . . . . 104 


\section{Abstract}

Research undertaken for this thesis aimed to detect and identify stellar variability in the database of the Japan/New Zealand MOA collaboration. The database of stars collected by the MOA project provided an extensive source of raw data for analysis. Detection of stellar variability was performed by several $\mathrm{C}++$ programs created by the author, which incorporated the Welch and Stetson variability index, the Schwarzenburg-Czerny period folding program, a microlensing modelling program and a transit detection program.

The search for stellar variability produced 83 Cepheid variables, 265 long period variables, 59 eclipsing binaries and 6 potential microlensing events. Sixteen potentially interesting variations that could correspond to planetary transits were also detected. The folded lightcurve of one of the potential transits was categorised as a 'very interesting transit' and 15 were categorised as 'interesting transits'. The search for planetary transits ultimately proved unsuccessful, however, a detailed statistical study of the MOA data revealed several alterations concerning observational procedures which could be made to optimise the MOA data for any future search for planetary transits. 


\section{Preface}

This thesis is the culmination of two years research undertaken as part of the MOA collaboration, which comprises numerous Japanese and New Zealand scientists and astronomers. Over the course of these two years, the author has been actively involved with the MOA project, and has undertaken several trips to Mt John University Observatory as part of the research programme. Research for this thesis comprised several aspects, including searching the archives of the MOA project for variable stars, microlensing events and planetary transits.

Another important research element was to test the software developed by Michael Reid. This involved running the Victoria University data reduction pipeline on real data collected by the MOA project at Mt John University Observatory. Once the pipeline output had been placed into the StarBase database, software was developed to identify variable stars.

During the course of this research, it became apparent that there had been little success in identifying planetary transits from ground-based observations. Consequently, a program which searched for these events was developed and it was used on suitable sets of images that had been reduced using Ian Bond's difference imaging software. Although the search was not fruitful, it demonstrated that it is possible to locate extrasolar planetary transit events from ground-based observations.

While a member of the MOA project the author has been involved in 4 publications.

1. Bond, I., ... Wilkinson, S., ... (and 28 others - the MOA collaboration). Study by MOA of extrasolar planets in gravitational microlensing events of high magnification, MNRAS (2002), 333, pp71-83.

2. Bond, I., ... Wilkinson, S., ... (and 20 others - the MOA collaboration). Improving the prospects for detecting extrasolar planets in gravitational microlensing events in 2002, MNRAS (2002), 331, ppL19-L23. 
3. Noda, S., ...Wilkinson, S., ... (and 32 others - the MOA collaboration). Study of variable stars in the MOA data base: long-period red variables in the Large Magellanic Cloud, MNRAS (2002), 330, pp137-152.

4. Bond, I., ...W Wilkinson, S., ... (and 28 others - the MOA collaboration). Real-time difference imaging analysis of MOA Galactic bulge observations during 2000, MNRAS (2002), 327, pp868-882. 


\section{Acknowledgements}

I would like to thank my supervisor Denis Sullivan for his help and guidance with a project and collaboration that I knew little about when I started. Denis was the first to introduce me to the wonderful world of astrophysics and I am particularly grateful for his insights into this project. His enthusiasm for the topic was highly infectious and I enjoyed our meetings, both formal and informal, as well as his great sense of humour.

I would also like to thank fellow student Mike Reid for providing me with the pipeline reduction system and the StarBase database, and for readily answering my many and varied questions. His help and friendship was invaluable and I am particularly thankful for his computer tuition, thesis editing and general knowledge about everything!

Thank you to all the members of the MOA project, especially Ian Bond, post-doctoral fellow, for encouraging me to go in new and interesting directions with my research, and Philip Yock, New Zealand project leader, for allowing me to be part of this international collaboration. I am indebted to the MOA project for their generous financial grant, which has aided in the production of this thesis.

Thanks also to Rob Lenihan for his valuable help on the computing front. For advice and support above and beyond the call, I would like to thank my friends and family. And finally, I would like to thank my partner, Catherine Field-Dodgson, for her editing and support. 


\section{Chapter 1}

\section{Introduction}

\subsection{MOA (Microlensing Observations in Astrophysics)}

The MOA collaboration was formed in 1995 with the objective of observing gravitational microlensing events. A joint Japanese and New Zealand collaboration, the project currently comprises academics, students and staff from Nagoya and Tohoku Universities in Japan, the University of Auckland, Victoria University of Wellington, Canterbury University in New Zealand, Mount John University Observatory (MJUO) and, until recently, Carter Observatory in New Zealand.

Gravitational microlensing refers to the deflection of light from a luminous (source) object by a massive lensing object which lies directly between the observer and the source object. The investigation of microlensing events was suggested as a possible method for detecting non-luminous or faint objects, which contribute to galactic halo dark matter. In order to detect these rare events, millions of stars must be observed over a time scale of hours to years. In recent years, microlensing has also become a method for detecting extrasolar planets. A detailed discussion of the history, applications, and physics of gravitational microlensing will be presented in chapter 4 .

\subsubsection{Mount John University Observatory}

The MOA project makes observations from MJUO, which is operated by the Department of Physics and Astronomy of the University of Canterbury. MJUO is located in the centre of the South Island of New Zealand and currently runs the three most powerful telescopes in New Zealand, including the telescope allocated for MOA observations.

An important quality for an observation site is the angular resolution or 'seeing conditions'. Astronomers use the term 'seeing conditions' to describe the effects of clear air 
MJUO Seeing 1992-1998

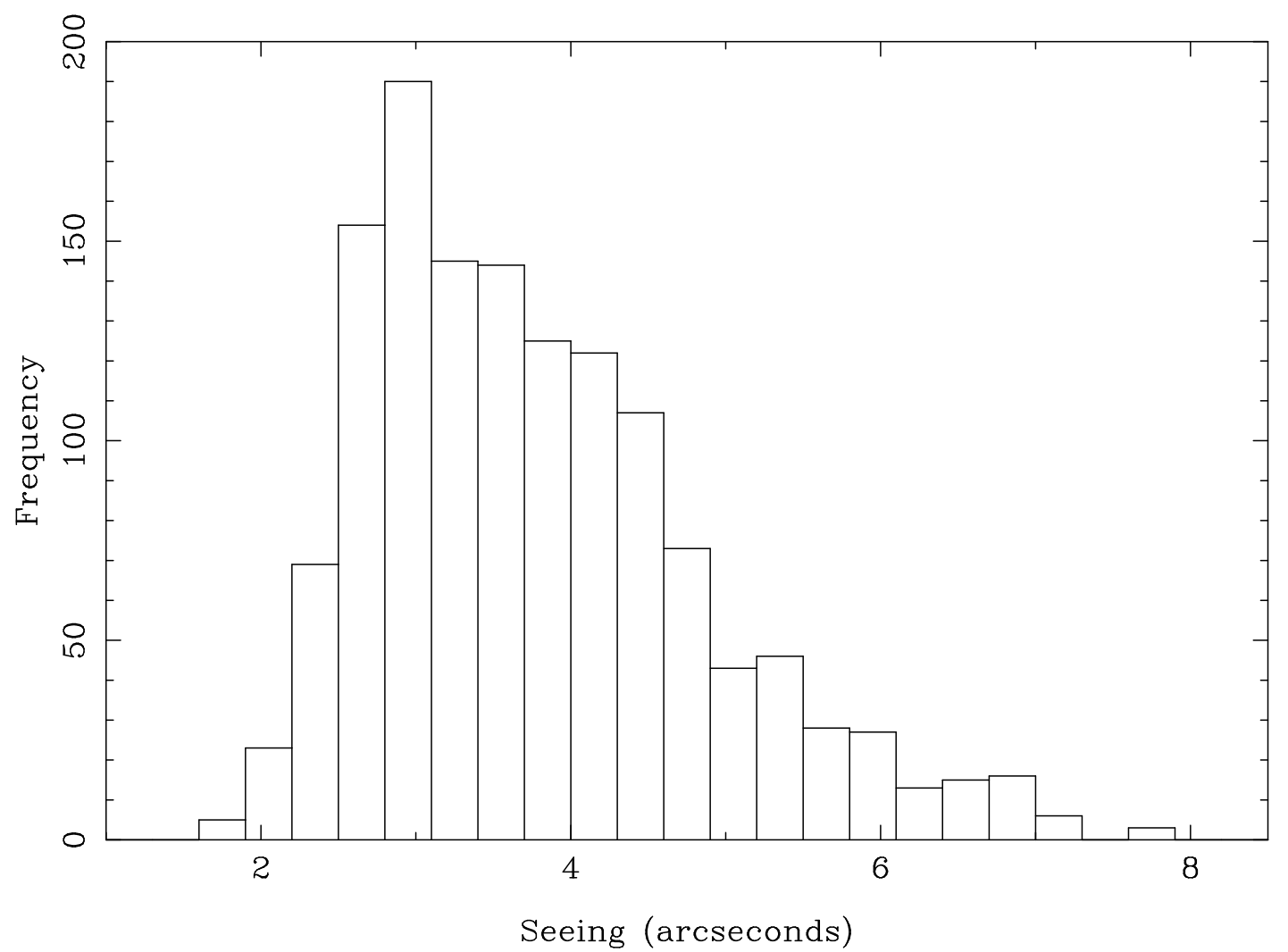

Figure 1.1: Seeing conditions at Mount John University Observatory from 1992 to 1998. (data courtesy of John Pritchard, University of Canterbury).

atmospheric turbulence on imaging, which causes rapid changes (up to $\sim 30 \mathrm{~Hz}$ ) in the apparent position of an object. Relatively long exposure observations record the resulting flux profile as an approximately Gaussian point spread function (PSF) centred on the position of the object. The Full Width at Half Maximum (FWHM) of the Gaussian flux distribution is used to estimate the seeing conditions measured in units of angular displacement. Sites with good seeing conditions are located at high altitudes, as this reduces the amount of atmosphere that light has to pass through in order to reach the telescope. Good seeing sites also have low levels of atmospheric turbulence in the atmosphere above the site; both low altitude and turbulent atmospheres have a detrimental effect on the quality of the observations. Seeing conditions at MJUO are rarely better than 2 arcseconds, but a significant fraction of clear nights are between 2 and 3 arcseconds (see figure 1.1).

\subsubsection{The MOA Boller \& Chivens telescope}

The MOA project's observations were made using a $61 \mathrm{~cm}$ Boller and Chivens (B\&C) cassegrain reflector located at MJUO. The telescope was originally purchased by MJUO 
in 1975. In 1997 the MOA collaboration increased the telescope's field of view by reducing the focal ratio from $f / 13.5$ to $f / 6.25$, and installed a vastly improved computer controlled guidance system.

\subsubsection{MOA-cam1}

The MOA-cam1 CCD camera was installed in 1996 and was used by the MOA project until 1998, when it was replaced with MOA-cam2. When MOA-cam1 was installed on the $\mathrm{B} \& \mathrm{C}$ telescope, the focal ratio of $f / 13.5$ produced a full field of view of $30^{\prime} \times 30^{\prime}$, with an individual pixel scale of $0.3 \mathrm{arcsec} /$ pixel. The telescope's focal ratio alteration from $f / 13.5$ to $f / 6.25$ changed the field of view to $0.97^{\circ} \times 0.98^{\circ}$, with a pixel scale of $0.65 \mathrm{arcsec} / \mathrm{pixel}$. This change in optics increased the field of view by a factor of four, increasing the number of stars observed in one observation by a similar factor.

Constructed and installed by Japanese members of the MOA project, MOA-cam1 comprised nine CCD chips in a $3 \times 3$ grid. The CCDs were Texas Instrument TC 215 CCD chips consisting of $1000 \times 1018,12 \times 12 \mu \mathrm{m}$ pixels. Each individual CCD chip was placed 800 pixels in right ascension and 812 pixels in declination from adjacent chips. To cover the area lost between the CCD chips, the CCD array was stepped through four positions each overlapping the previous position by 100 pixels. The nine $1000 \times$ 1018 CCDs stepped through the four positions resulted in a mosaic of 36 CCD images (figure 1.2). Typically a single 36-frame set observed towards the Large Magellanic Cloud (LMC) would contain approximately 400,000 stars.

\subsubsection{MOA-cam2}

In 1998 a new CCD camera, MOA-cam2, replaced MOA-cam1. MOA-cam2 consists of three individual CCDs which were aligned parallel to and connected to the adjacent CCDs. The CCDs were abutted, thinned, back illuminated, Scientific Imaging Technologies (SITe) ST-002A CCD chips consisting of $2048 \times 4096,15 \times 15 \mu \mathrm{m}$ pixels (Yanagisawa et al. 2000). The alignment of the CCDs in a single row (figure 1.3) results in a total area of $6144 \times 4096$ pixels. However, the three CCDs have a small area of 'dead space', between the active areas of the CCD chips of $100 \mu \mathrm{m}$ ( $\sim 7$ pixels). The MOA-cam2 CCDs combined with the B \& $\mathrm{C}$ telescope and $f / 6.25$ optics results in a full field of view for the three chips of $0.92^{\circ} \times 1.39^{\circ}$ from individual pixels of $0.81 \mathrm{arcsec} /$ pixel. 


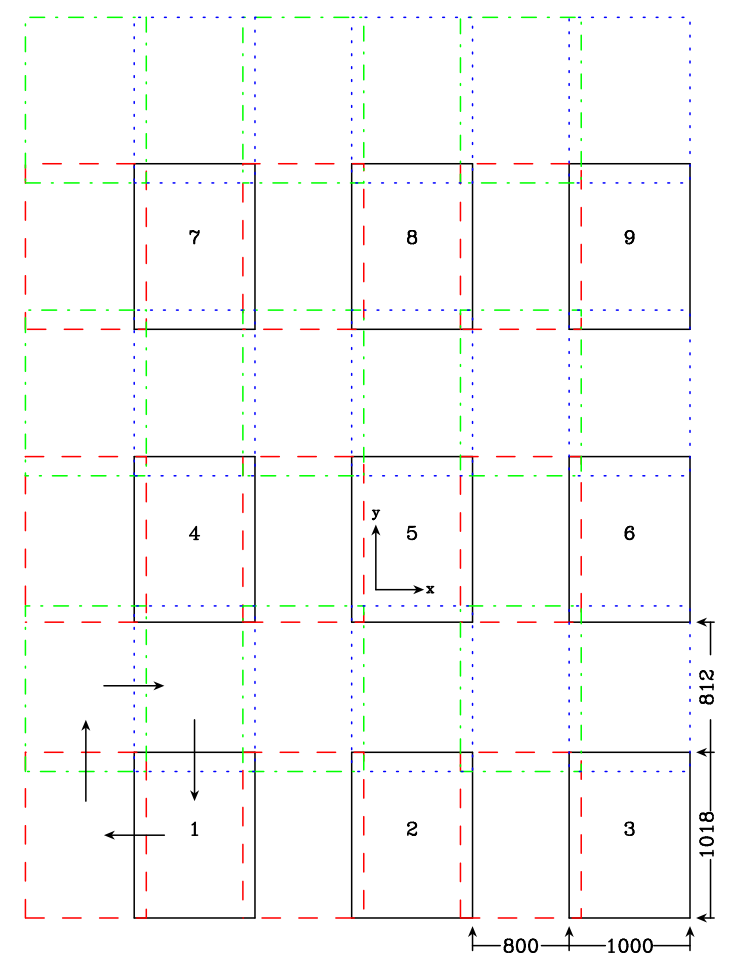

Figure 1.2: This figure shows the layout of the nine CCDs that make up MOA-cam1 and how they are stepped through four positions to cover the target area. All distances are given in pixel widths. The vertical and horizontal scales of the this figure differ by approximately $25 \%$ in order to emphasise the difference in size of the vertical and horizontal axes. Credit Michael Reid (Reid 2002), reproduced with permission

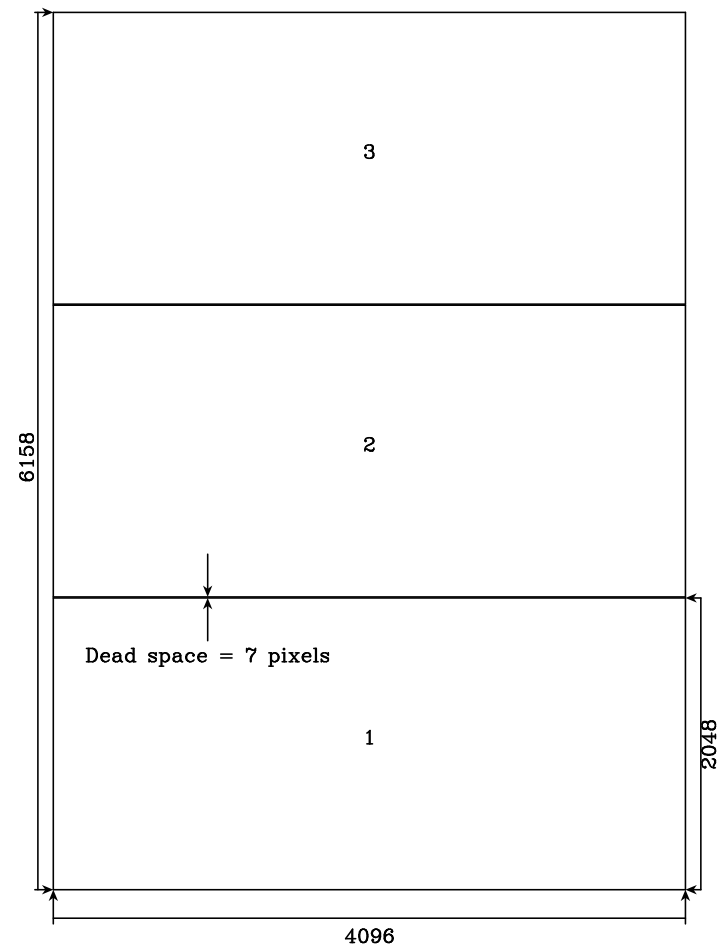

Figure 1.3: This figure shows the layout of the three CCDs that make up MOA-cam2. All distances are given in pixel widths. Adapted from Michael Reid (Reid 2002), reproduced with permission 


\subsubsection{MOA filters}

Observations made by the MOA project were collected by observing through one of two custom-made ultra-broadband filters. The MOA-Blue filter covers wavelengths from $400-630 \mathrm{~nm}$ and the MOA-Red filter covers wavelengths from $630-1000 \mathrm{~nm}$.

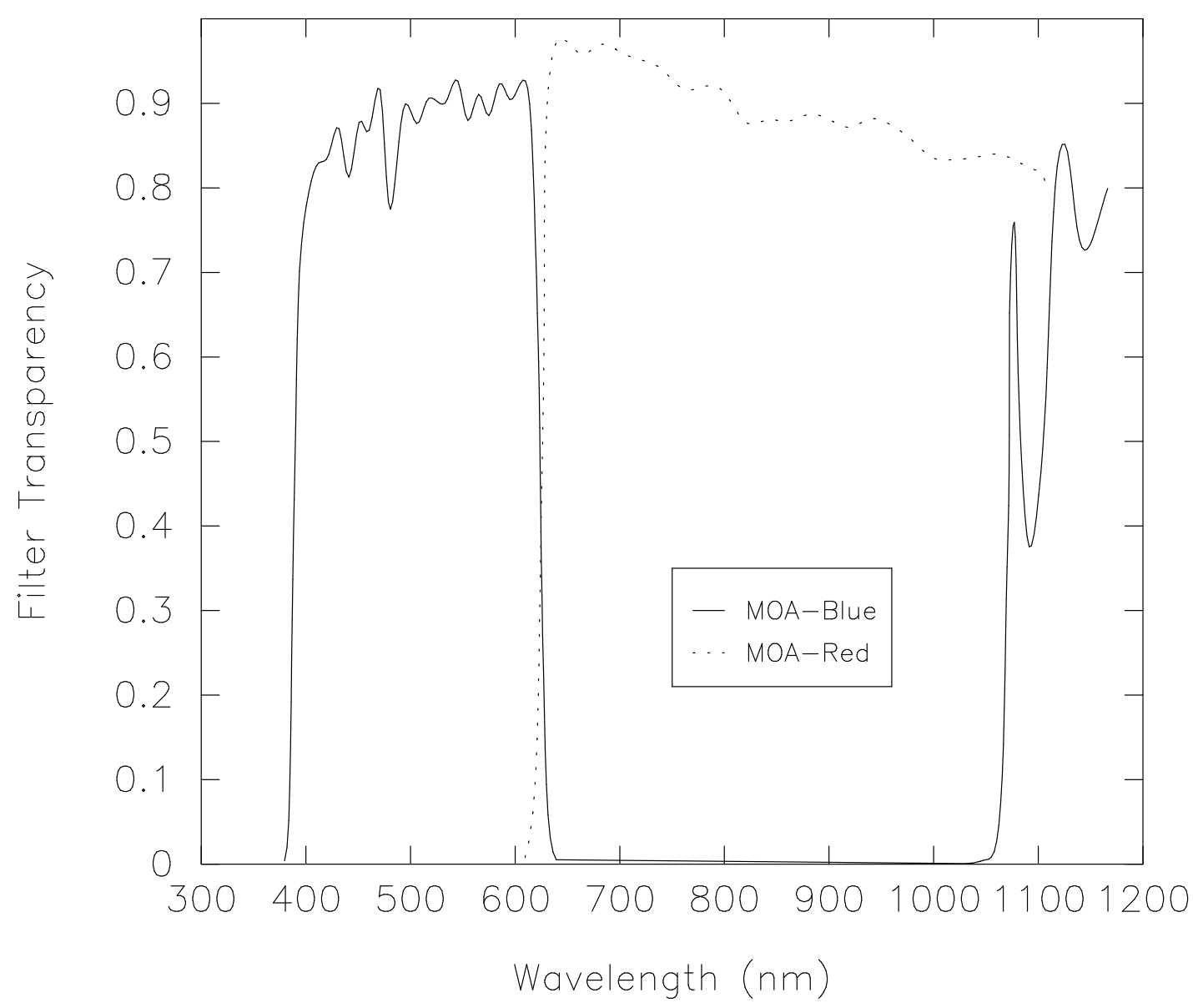

Figure 1.4: The figure shows the transmission for both of the MOA filter as a function of wavelength.

\subsubsection{Observation fields}

For approximately 8 months of the year, the MOA project targets the Galactic Bulge when it has attained sufficient altitude for observational purposes. MOA also targets both the LMC and Small Magellanic Cloud (SMC). Because of the southern latitude of Mount John University Observatory ( $43^{\circ} 59.2^{\prime} \mathrm{S}$ ), the MOA project is able to usefully observe the LMC and SMC for the entire calendar year. The first combination of telescope (focal ratio f/13.5) and camera (MOA-cam1) observed three LMC fields, while the second combination of telescope (focal ratio f/6.25) and camera (MOA-cam1) observed three fields in the LMC and two fields in the SMC. To date, the combination of telescope (f/6.25) and camera (MOA-cam2) used by the MOA project observes sixteen LMC fields, 

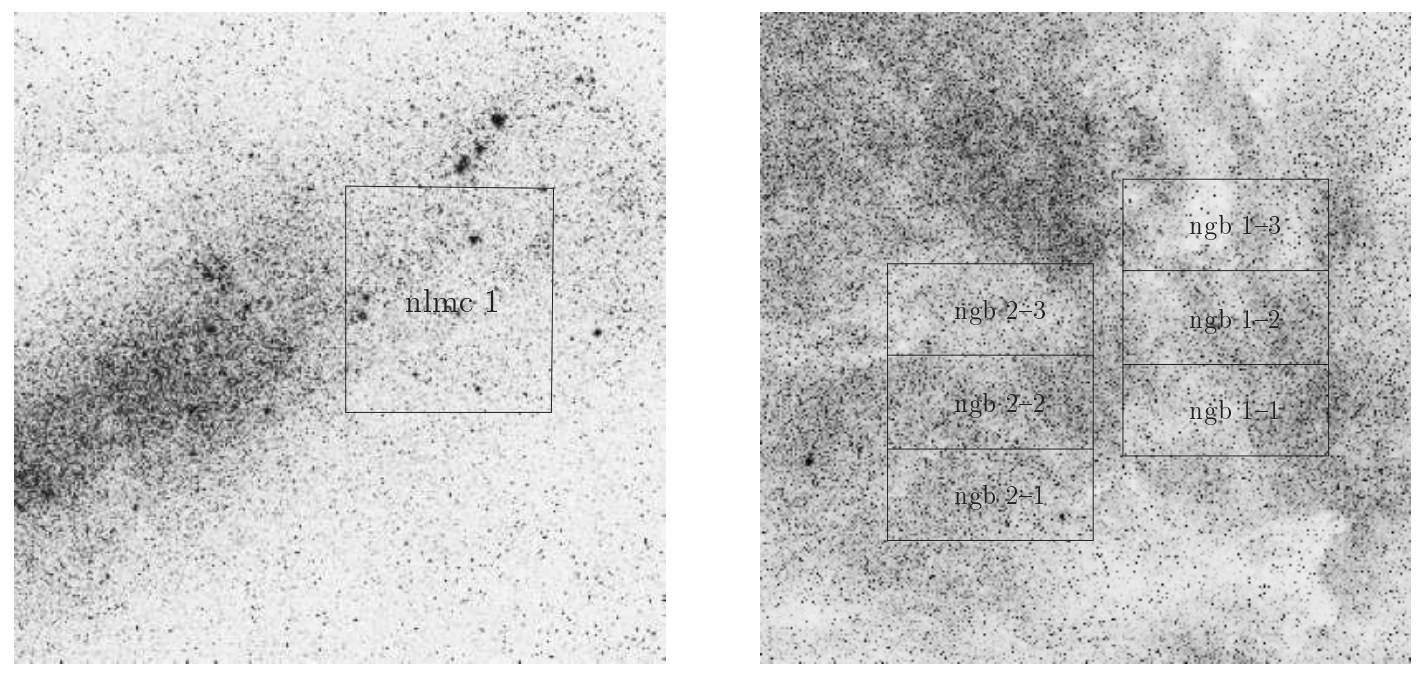

Figure 1.5: The position of the $1997 \mathrm{nlmc} 1$ and $2000 \mathrm{ngb} 1$ and ngb 2 fields are shown against the background images taken from the ESO online digitized sky survey. In the image on the right the approximate positions of the individual MOA-cam2 CCDs are shown.

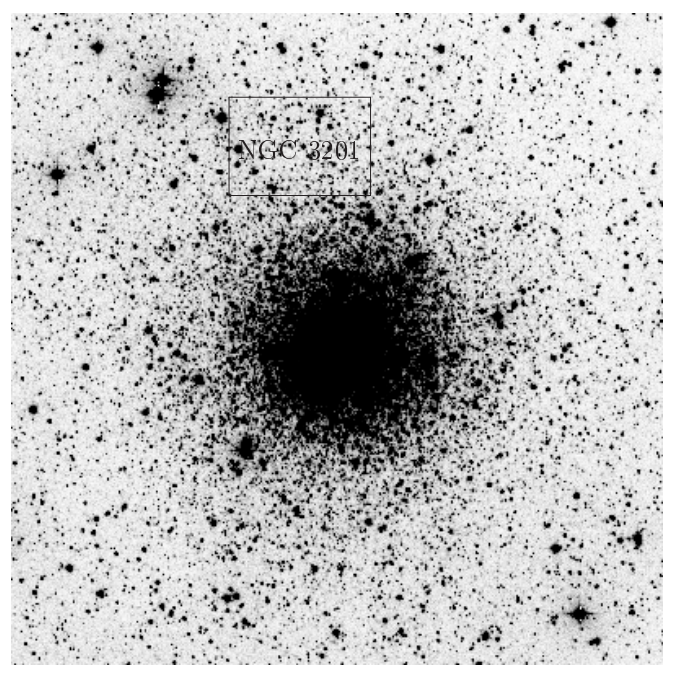

Figure 1.6: The position of the NGC 3201 calibration field is shown against the background image of the NGC 3201 globular cluster taken by the MOA project. This is the region given by Alcaimo et al (1989). 
eight SMC fields, and fourteen Galactic Bulge fields. In addition to observing the standard MOA fields, the MOA project has also made observations of the globular cluster NGC 3201, which were used to calibrate the MOA data. MOA also observes other targets of opportunity such as microlensing events detected by similar projects and Gamma Ray Bursts (GRBs).

\subsubsection{Calibration}

When a single star is observed with different instruments in different locations, the two resulting data sets cannot be compared until they have been calibrated. Calibrations investigate how the detection instruments and atmospheric extinction affect the data collected at any given site. In order to perform a calibration, 'standard' stars with known properties need to be observed. The results of these observations are compared to earlier observational data which used standard photometric filters and detection instruments. The differences between the current non-standard observations and the so-called standard observations are used to calculate photometric transformations, which enable estimates of the observations that would have been made using standard filters and instruments.

\subsubsection{Data storage}

Observations made with the MOA CCD camera are stored as FITS (Flexible Image Transport System) images (Wells, Gresien \& Harten 1981). Astronomical data is stored in the FITS format as a multidimensional binary array with an ASCII text header containing approved keywords. The ASCII text header was included in the FITS format so that information about the data could be transferred with the array and easily interpreted. FITS image headers are in the format

$$
\text { Keyword }=\text { value } / \text { comment }
$$

The FITS format was established as a standard when it was endorsed by the International Astronomical Union in 1982, and it was created in such a way that modifications could be made, so that the format could adapt readily to new astronomical developments.

A MOA 2048 × 4096 pixel Galactic Bulge image contains approximately 200,000 stars, and creates an approximately 16 Mb FITS image. Each image from one night of observation is stored on a Sun Microsystems e450 UltraSPARC II computer with $300 \mathrm{~Gb}$ of disk space. Recent observations are stored on the same computer until $40 \mathrm{~Gb}$ of compressed 
data $^{1}$ have been acquired, when it is then copied to Digital Linear Tape (DLT). The MOA project currently holds $>40$ DLTs (corresponding to $>100000$ images) in archive storage.

\footnotetext{
${ }^{1}$ Raw images have their physical memory size reduced by using a compression program. The MOA project uses the GNU gzip program found on UNIX systems.
} 


\section{Chapter 2}

\section{The MOA CCD data reduction pipeline}

\section{$2.1 \quad$ Astronomical measurement}

\subsubsection{Brightness}

The astronomical magnitude scale was first introduced by the Greek astronomer Hipparchus in 129 B.C., in order to compare the brightness of various stellar objects. The original scale ranged from stars 'of the first magnitude' (brightest) to stars 'of the sixth magnitude' (dimmest). The Italian astronomer Galileo's invention of the telescope in 1610 allowed stars fainter than sixth magnitude to be observed, therefore, the magnitude scale was extended to stars 'of the seventh magnitude'. As telescope technology advanced, fainter stars were able to be observed and the magnitude scale was again extended further. In 1856, Norman Pogson, an Oxford astronomer, formally defined the logarithmic magnitude scale by equating a difference of five magnitudes to a brightness ratio of a 100. This magnitude scale describes the apparent magnitude of an object as seen from the Earth but gives no indication of distance. Introducing the absolute magnitude scale normalized the brightness of stellar objects with respect to distance. The absolute magnitude of an object is its apparent magnitude measured from a standard observer distance of 10 parsec (32.6 lightyears).

An astronomical object's brightness is also measured in flux (for point sources); it has the physical units of Watts per square meter and measures the energy per unit time of electromagnetic radiation which passes through a unit perpendicular area. The apparent magnitude difference between two objects $\Delta m$, is related to this flux difference by the equation 


$$
\Delta m=m_{1}-m_{2}=-2.5 \log _{10}\left(f_{1} / f_{2}\right)
$$

where $m_{1}$ and $f_{1}$ is the apparent magnitude and flux, respectively, of the measured object and $m_{2}$ and $f_{2}$ is the apparent magnitude and flux of a comparison object.

\subsubsection{Time}

A standard measure of time in astronomy is the Julian day, in which a single Julian day is equal to one Earth solar day (24 hours). The Julian day is a continuous count of the number of days since Julian day 0 , which began at noon on the $24^{\text {th }}$ of November $-4713^{1}$ at Greenwich, England, (an arbitrary date, but sufficiently far back in time to cover all recorded astronomical phenomena) and is an integer number that equals the number of whole days elapsed since Julian day 0 . The Julian Date (JD) equals the number of whole days, plus the fraction of the day past when the time was recorded. It is advantageous to utilise the Julian Date system instead of a conventional time recording system, because it is a continuous record which makes it easy to use in mathematical equations. One advantage of using Julian Dates is a new Julian day begins at noon Greenwich Mean Time (GMT), which is during daylight hours (for European observers) and not at night, when most observing takes place.

However, the Julian Date system has its disadvantages too, the primary one being that the continuous count of days from Julian day 0 produces a large number to the current epoch, which results in digit overflow when using single precision storage in most computer programming languages. Moreover, as the MOA project operates from Mt John in New Zealand, which is approximately half a day ahead of GMT, a new Julian day begins at midnight, when most observing takes place. In order to alleviate these problems, the MOA project resorted to using the Modified Julian Date (MJD), which is related to the Julian Date (JD) by $M J D=J D-2400000.5$.

\subsection{Data reduction}

The process of data reduction converts astronomical observations into results for analysis and interpretation. Data are collected by the MOA project by means of a telescope and CCD system which produces digital images, that are stored in the standard FITS format. Data reduction for the MOA project requires transforming digital images into

\footnotetext{
${ }^{1}$ Proleptic Julian calendar.
} 
brightness (and its uncertainty) and position measurements for each of the objects found in the image. The MOA project runs a real time search for microlensing events toward the Galactic Bulge (Bond et al. 2001), which requires fast reduction of multiple images. Follow up analysis of the MOA data searches for extrasolar planets, which requires easily accessible accurate data ${ }^{2}$.

\subsubsection{DAOphot}

The DAOphot image reduction software was created by Canadian astronomer Peter Stetson, and uses Point Spread Function (PSF) fitting to model objects from CCD images (Stetson 1987). The program searches for stellar objects by fitting a Gaussian PSF to every obvious stellar image in the image and a table of numerical values are used to represent how well the Gaussian function fits the area surrounding the selected pixels. If the numerical value suggests a good fit, the brightness of the object is calculated from the height of the Gaussian function at its centre. Fitted objects are then subtracted from the image and passed through DAOphot again to search for fainter objects. Images from the calibration field NGC 3201 were reduced using DAOphot II software (figure 3.2).

\subsubsection{DoPHOT}

PSF fitting is also used by DoPHOT, in order to calculate the brightness and shape of the objects observed. The DoPHOT data reduction program was originally developed by astronomers Paul Schechter, Mario Mateo and Abi Saha for the purpose of classifying objects as stars and galaxies, and to collect stellar photometry using non-regular PSFs (Schechter, Mateo \& Saha 1993). DoPHOT fits objects to a model with six parameters: $\mathrm{x}$ and $\mathrm{y}$ position, total intensity and the shape parameters of height, width and tilt. By fitting the model to objects above a high threshold value, the typical shape of objects in the image are able to be determined. The fitted model is then subtracted from the image and the fitting of objects above a lower threshold begins. This process continues until all identified objects in the image have been modelled and subtracted. Images from the LMC collected during 1997 were reduced using the DoPHOT reduction software. DoPHOT is significantly faster than DAOphot II, but usually makes less accurate measurements in crowded fields, primarily due to the less sophisticated PSF used.

\footnotetext{
${ }^{2}$ Searching for extrasolar planets using the microlensing and planetary transit methods requires photometry with deviations in brightness of less than $1 \%$
} 


\subsubsection{Difference imaging}

Difference imaging is the process of subtracting two images and investigating the subtracted image for any residual differences. The first of the two images is a reference image which is created by 'stacking' a series of images collected during good seeing conditions. The reference image sets the base level magnitude values for all the objects found in the image and all subsequent measurements of brightness are relative to the reference image. Images collected separately from the reference image are related to the reference image by

$$
i(x, y)=r(x, y) \otimes k(x, y)+b(x, y)
$$

where $i$ is the separately collected (current) image and $r$ is the reference image. The difference in the seeing conditions between the two images is represented by the convolution kernel $k$, and $b$ the difference in background values. The $\otimes$ symbol represents the convolution. When the current image is subtracted from the reference image with a correctly calculated convolution kernel, in theory any constant stars will be removed leaving only those stars which have varied in brightness. A version of difference imaging (designed by Ian Bond of the MOA project) is currently being used at MJUO to provide real-time analysis of MOA images from the Galactic Bulge (Bond et al. 2001). Data gathered from the MOA Galactic Bulge fields were used in the search for planetary transits (described in section 5.3.2). The CCD frames were reduced on site using the difference imaging procedure.

\subsubsection{The Victoria University Wellington data reduction pipeline}

A MOA data reduction pipeline has been developed for the MOA project by Michael Reid of Victoria University Wellington and has been available for use since April 2000 (Reid 2002). It was designed to be portable, easy to use and to produce high quality photometry. The pipeline consists of a PERL 'wrapper' script that calls programs written in $\mathrm{C}$ and $\mathrm{C}++$. Reduction of data using the pipeline begins by running the PERL wrapper program (pipeline.pl) with the name of a file containing a list of the images to be reduced as the only argument. The pipeline consists of a series of processes which reduces the input FITS images to photometry stored in a database. The first process of the pipeline checks the FITS image header for the correct keywords, and inserts the correct keywords if if they are not found. Following this step, the images are 'flatfielded', which removes any pixel-dependent gain discrepancies introduced by the instruments during the collection of the images. Smaller sub-images are then created from the original 2048 
$\times 4096$ pixel FITS images $(1000 \times 1000$ pixels is the default size $)$, and are reduced ${ }^{3}$ into text photometry files which contain brightness, uncertainty in brightness and position information for every object found in the sub-image. The text files for each sub-image are combined to create a photometry file of the original FITS image. The final process of the pipeline archives the photometry in a database format that can be accessed using a $\mathrm{C}++$ extraction program. Each of the processes in the data reduction pipeline can be run as individual programs or they can be run collectively using the pipeline.pl wrapper script.

When an image is reduced by the pipeline, two text files are produced which contain the image's data. Brightness, error, and position values for each object found in the image are stored in the <image_name> . out file, while the <image_name>. info file stores information such as the start time of the observation, the image's field name and the name of the filter through which the starfield was observed ${ }^{4}$. A new database system (StarBase) was designed as part of the data reduction pipeline for archiving MOA data. Both the .out and .info files produced by the pipeline's image reduction stage are plain text files, which allows observations in different formats to be converted into .out and .info files and stored in StarBase.

\subsection{Analysis tools for reduced data}

The StarBase database was also developed by Michael Reid of Victoria University Wellington (2002) to record data that was produced from the MOA observations. StarBase was designed so that data could be accessed using a basic $\mathrm{C}++$ extraction program, which included functions needed to retrieve time (Julian Date), brightness (magnitude), the uncertainty in brightness ( $\sigma$ magnitude) and the filter information for observations of every star recorded in the database. Information retrieved by the extraction program was stored in data arrays that could be accessed for further analysis by other separate functions and programs. As part of the extraction program, brightness data for each star was converted from magnitude to flux and $\sigma$ magnitude to $\sigma$ flux, and time data was converted from Julian Date to the Modified Julian Date. A separate function calculated the mean, median and standard deviation values of the brightness from the data arrays created by the extraction program. This method of extraction meant that using further

\footnotetext{
${ }^{3}$ The data reduction pipeline allows the use of DAOphot II, DoPHOT or difference imaging for the reduction of the sub-images.

${ }^{4}$ Examples of the .out and .info files produced by the pipeline's image reduction are shown in appendix A.1
} 
analytical tools which made use of the calculated values and data arrays, could be easily added to the extraction program. 


\title{
Chapter 3
}

\section{Miscellaneous variable stars}

\subsection{Analysis tools used in searching for variable stars}

\begin{abstract}
All stars display variations of brightness and colour in the course of their passage through subsequent stages of stellar evolution. As a rule, however, a star is called variable when the brightness or colour variations are detectable on the time scales of the order of the mean life time of man. ${ }^{1}$
\end{abstract}

For a microlensing survey to be successful, it is necessary to observe millions of stars over the time scale of years. A notable percentage of stars are known to vary significantly in brightness over time scales from minutes to years. Microlensing surveys monitor large numbers of stars, therefore numerous variable stars have been detected during the course of microlensing projects. Paczyński's 1986 paper, 'Gravitational microlensing by the galactic halo', described a method for observing microlensing events, and noted that new variable stars would be discovered using the microlensing survey method that he suggested. Indeed, it is now commonly believed that microlensing projects have discovered more variable stars than ever before.

\subsubsection{Variability index}

The Welch and Stetson variability index was designed for photometry collected with one or two filters, and obtained at many epochs (Welch \& Stetson 1993). It produces a numerical value to represent the degree of brightness variation, which occurs in the object's lightcurve. The index $(I)$ is calculated from the equation

\footnotetext{
${ }^{1}$ Sterken \& Jaschek 1996.
} 


$$
I=\sqrt{\frac{n}{n-1}} \frac{1}{n} \sum_{i=1}^{n}\left(\frac{b_{i}-\bar{b}}{\sigma_{b, i}}\right)\left(\frac{v_{i}-\bar{v}}{\sigma_{v, i}}\right)
$$

where $b_{i}$ and $v_{i}$ are the magnitude measurements from two observations of the same star, closely spaced in time on some occasion $i$, and $\sigma_{b, i}$ and $\sigma_{v, i}$ are the respective uncertainty values for the observations $b_{i}$ and $v_{i}$. The variables $\bar{b}$ and $\bar{v}$ are the calculated mean values of the magnitude for the total number of $n$ observations.

The index was used in two different ways for the analysis of the MOA data. Data collected from the 1997 MOA LMC fields contained approximately equal numbers of observations taken through the MOA-red and MOA-blue filters. The data acquisition process recorded all the fields of one 36 image mosaic observed through the MOA-red filter, and then recorded the same fields observed through the MOA-blue filter. These two sets of observations were assigned as one pair for the Welch and Stetson variability index, where $b_{i}$ is the MOA-blue magnitude and $v_{i}$ is the MOA-red magnitude. Data collected from the 2000 Galactic Bulge fields contained approximately 10\% MOA-blue observations and approximately $90 \%$ MOA-red observations. ${ }^{2}$ Therefore, the index only utilises the MOA-red observations by making successive MOA-red observations one pair, where $b_{i}$ is the MOA-red magnitude and $v_{i}$ is $b_{i+1}$.

According to Stetson (1996) this variability index requires that there be "two observations closely spaced in time on some occasion $i$ ". Data collected from both the 1997 LMC and 2000 Galactic Bulge fields contained observations separated by time intervals ranging from one hour to several months. These variations in the time intervals were caused by daylight hours (approximately 12 hours), full moons (approximately seven days) or technical problems with the observational equipment (which ranged from one day to several months). Consequently, observational pairs entered into the Welch and Stetson variable index were restricted to those separated by less than one day. The calculation of the variability index was incorporated as part of the author's C++ extraction program and was also used by a separate program that searched for variable stars as a reference for likely candidates.

\footnotetext{
${ }^{2}$ The percentage of MOA-red and MOA-blue observations was calculated from the 2000 Galactic Bulge field ngb 1-1.
} 


\subsubsection{Colour magnitude diagrams}

A colour magnitude diagram is a tool often used by astronomers, as under suitable circumstance it approximates to the Hertzsprung-Russell diagram (H-R diagram). The HR diagram was first conceived in 1911 by the Danish astronomer Ejnar Hertzsprung and, independently in 1913, by the American astronomer Henry Norris Russell. H-R diagrams show the correlation between total stellar energy output (usually the total photon energy) and the surface temperature of the star. In astronomical literature this can appear in a variety of forms such as absolute magnitude versus surface temperature, absolute magnitude versus spectral type or a colour magnitude diagram. Absolute magnitude is the astronomical magnitude of an object at a standard distance (10 parsecs) while the H-R diagram convention is to use a non-linear inverted temperature scale (which has its origins in using the spectral index of a stars as a temperature indicator). Stars displayed on the H-R diagram are not randomly distributed, but fall into well-defined groups. Each group represents a different stage of stellar evolution and consists of the main sequence, red giants, red and blue super giants and white dwarfs. The Colour Magnitude Diagram (CMD) attempts to approximate the H-R diagram by plotting the instrumental magnitude or flux versus the colour index.

Observations collected through different wavelength filters, such as red and blue filters, can be compared in order to gauge the colour index of a star; when the difference in brightness between these observations is calculated the colour can be determined. Because the MOA project uses both red and blue broadband filters, the colour index was calculated by subtracting the MOA-red magnitudes from the MOA-blue magnitudes. The instrumental colour index is a direct indicator of the stellar surface temperature due to the fact that the stellar surface is approximately a black body emitter; but the instrumental magnitude contains both stellar luminosity and distance information. Thus, stars displayed on the CMD will only approximate the H-R diagram if all the stars included in the diagram are the same distance from the observer.

The MOA project targets fields in the LMC, SMC and the globular cluster NGC 3201. All the stars observed within these fields are located at approximately the same distance to the Earth as the surrounding stars in each field, therefore, CMDs of these fields can be created and used as analytical tools. The MOA project also targets fields towards the Galactic Bulge; however, as many of the stars are located on the spiral arms as well as in the Bulge itself, the stars vary in distance. The CMDs that were produced for this thesis were obtained from images of the LMC and the globular cluster NGC 3201. The 


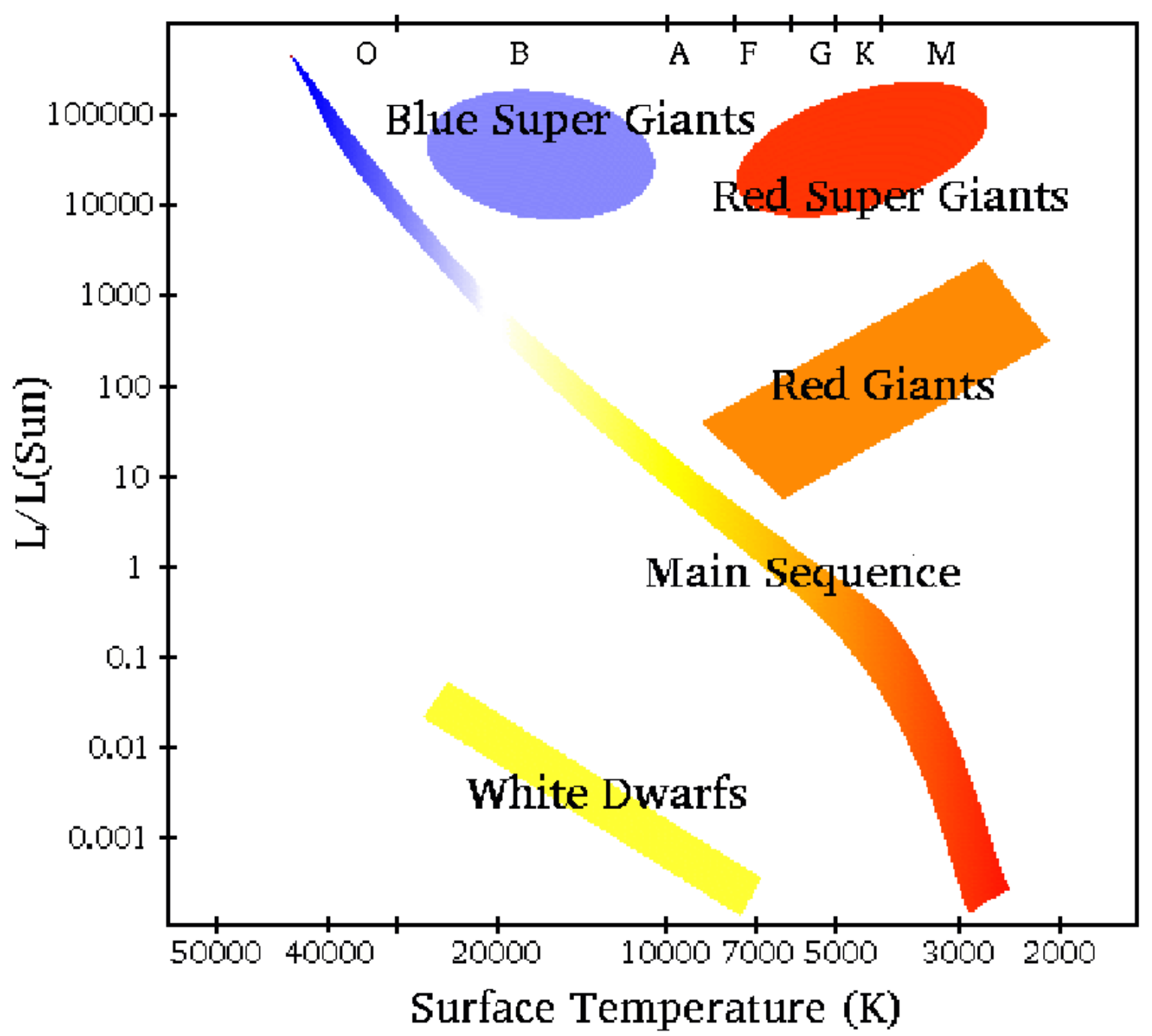

Figure 3.1: The H-R diagram shows the majority of stars appear on the main sequence of the diagram, with other groups of stars in the white dwarf, red giant and red and blue super giant areas.

globular cluster NGC 3201 is closer to the Earth than the $\mathrm{LMC}^{3}$, thus stars with fainter absolute magnitudes were able to be detected. Because of the large distance between the Earth and the LMC, only the brighter stars in the giant branch and the top of the main sequence were able to be detected in the LMC fields (figure 3.3).

Figure 3.2 shows the CMD of the globular cluster NGC 3201. The main sequence of stars can be seen at the bottom of the diagram and they extend up to approximately $14^{\text {th }}$ magnitude in MOA-red. Stars that are brighter than $14^{\text {th }}$ magnitude belong to the subgiant branch, which turns back toward the main sequence and the instability strip

\footnotetext{
${ }^{3}$ The distances from the Earth to the LMC and the globular cluster NGC 3201 are $55 \mathrm{kpc}$ (Groenewegen \& Oudmaijer 2000) and $5.2 \mathrm{kpc}$ (Harris 1996) respectively, where kpc = 1000 parsecs.
} 


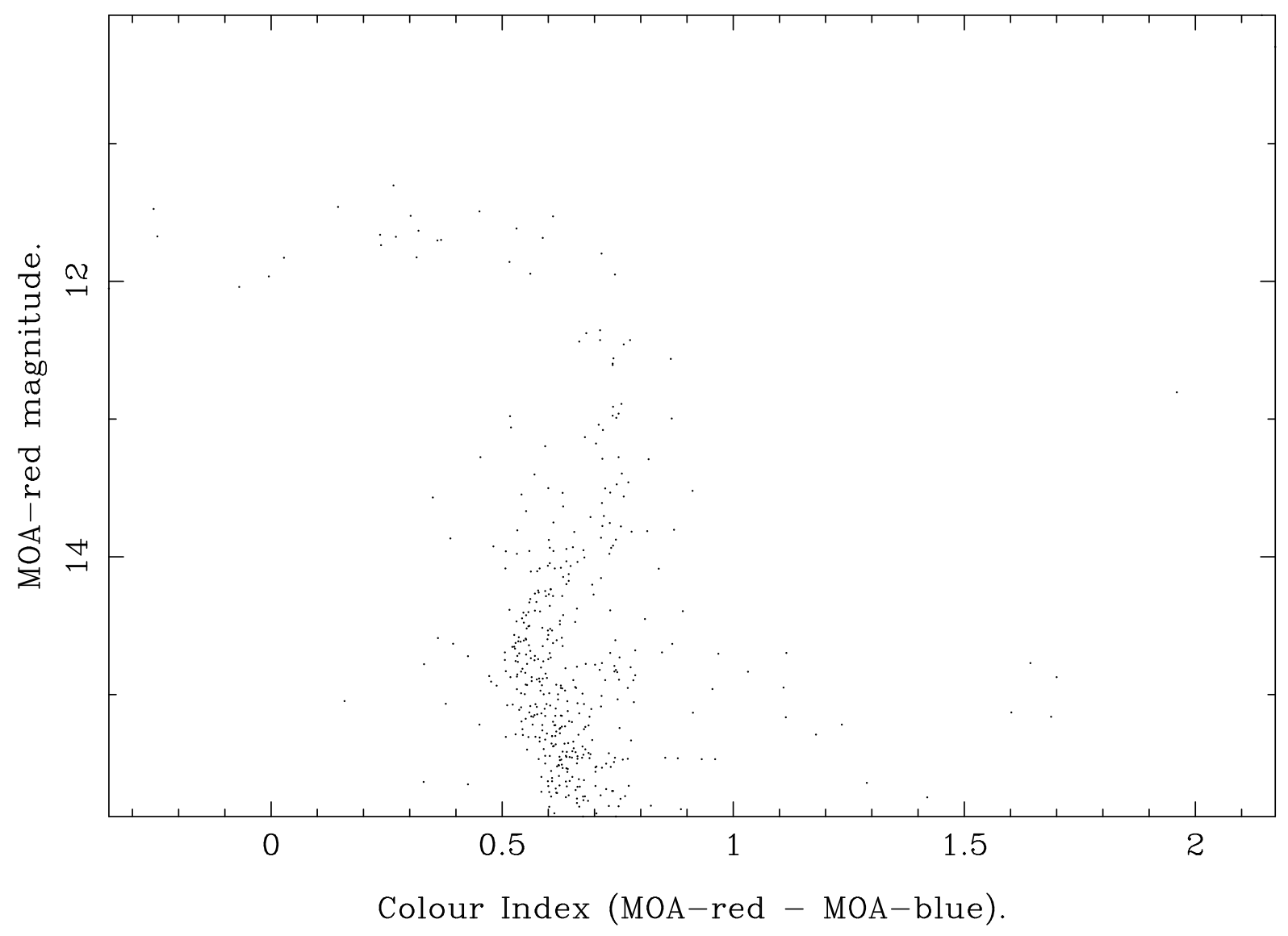

Figure 3.2: Colour magnitude diagram of the globular cluster NGC 3201. The diagram shows the main sequence turning off into the red giant branch, which then curves back toward the instability strip.

above the $12^{\text {th }}$ magnitude. Because of the large number of stars in each field, the CMDs could not be used directly to search for variable stars, but they could be used to identify the nature of the variable stars that were found. Long period variable stars are stars in the red giant stage of stellar evolution which are located in a unique position on the CMD. Figure 3.3 shows all the long period variable stars which are positioned in the giant branch of the MOA field nlmc 0-1. Cepheid variable stars occupy an area of the CMD which is referred to as the instability strip; this is located in line with the main sequence and level with the top of the giant branch. Figure 3.3 shows the MOA field nlmc 0-1 Cepheid variables clustered in an area of the CMD, which suggests the location of the instability strip.

The values required for producing a CMD were calculated by a function which was added to the $\mathrm{C}++$ extraction program. This function calculated the colour difference by using the mean values of the MOA-red and MOA-blue observations which were previously determined by the extraction program. The mean magnitudes and mean colour difference values for each star were stored in data arrays and written to a file called Colour<filter_colour $\rangle$ <field_name $>$.dat (described in appendix A.2) and then plot- 


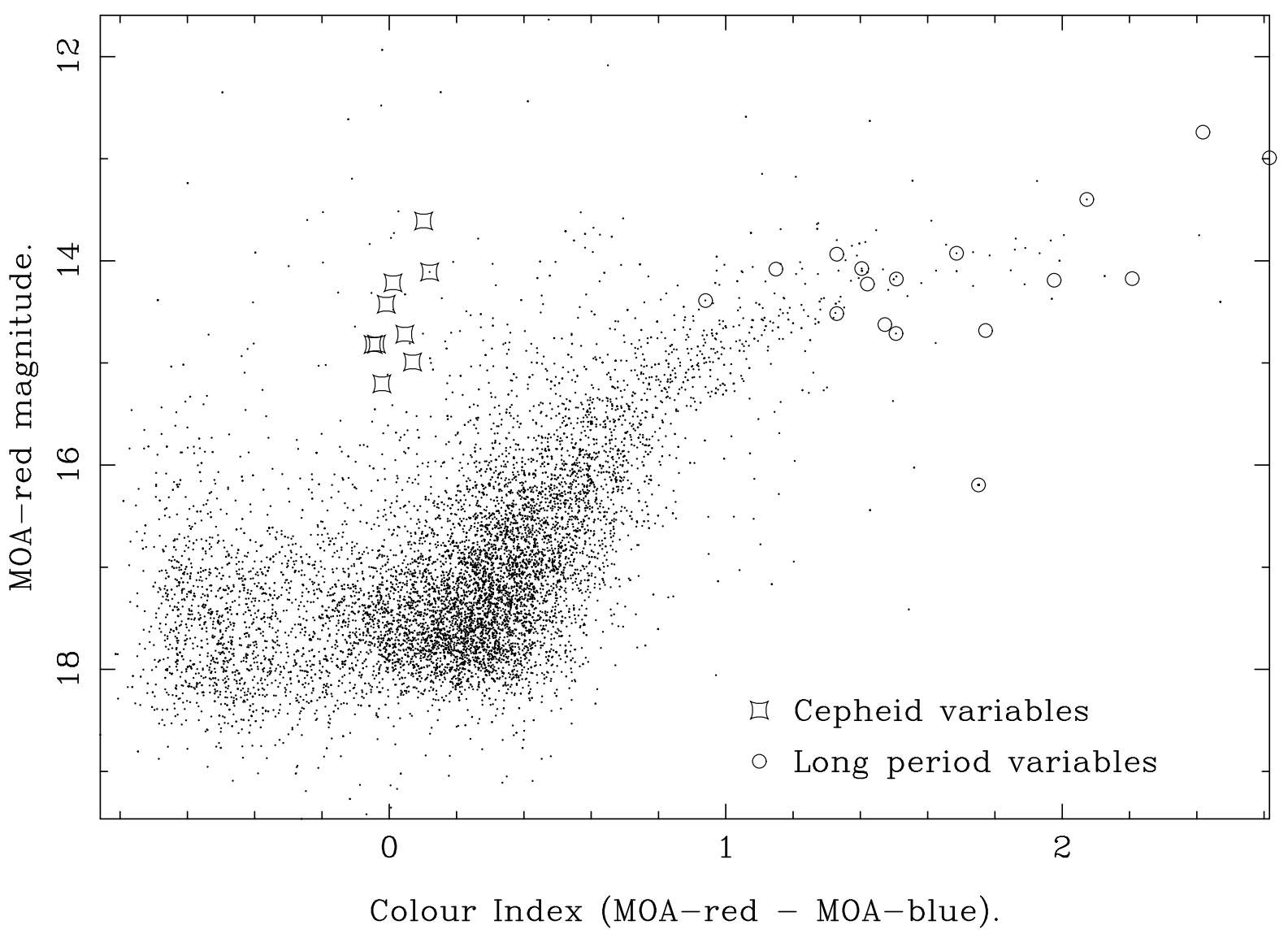

Figure 3.3: Colour magnitude diagram of the MOA LMC field nlmc $0-1$. The diagram shows the position of variable stars found in the field on the CMD .

ted by a PERL plotting script which uses the program suite PGPERL.

\subsubsection{Period folding for periodic variables}

The primary tool used in the search for periodic variable stars was the period folding program designed by Polish astrophysicist Alex Schwarzenberg-Czerny (SchwarzenbergCzerny 1996). It was designed for unevenly distributed observations and used the Analysisof-Variance (ANOVA) value to judge the quality of a fit. The original version of SchwarzenbergCzerny's period folding program was downloaded as several C program files, which were converted to $\mathrm{C}^{++}$and used together with the $\mathrm{C}^{++}$extraction program. Data arrays created for each star in the $\mathrm{C}^{++}$extraction program were passed through the Schwarzenberg-Czerny period folding program, provided they had a variable index above a minimum threshold limit. ${ }^{4}$ An ANOVA value was calculated for each star analysed by the period folding program and a minimum threshold level was placed on the

\footnotetext{
${ }^{4}$ The lower threshold limit for the variable index was adjusted for different fields, to remove stars with low values of the variable index (bottom $\sim 90 \%$ of indices).
} 
values. Consequently, stars which were unlikely to be variables were removed. All stars with ANOVA values above the threshold were written to an output file named lightcurve<Star_number>.dat, and searched by eye for periodic signals when the extraction program had retrieved all the recorded stars in the field. During the examination of the lightcurves, the user is prompted to classify the lightcurve into 'long period variables', 'Cepheid variables', 'eclipsing binaries' and 'unlikely variables'. Long period, Cepheid and eclipsing variables were saved to disk with appropriate file names ${ }^{5}$, while lightcurves which were classified as unlikely variables were deleted (an example of the lightcurve file format is shown in appendix A.2.2).

\subsection{Cepheid variables}

Cepheid variables are a class of variable star and were first discovered by John Goodricke, an English Astronomer, while he observed the star $\delta$ Cephei (after which the class is named). The American astronomer Harlow Shapley (Shapley 1914) was the first to suggest that the variability in Cepheid stars was caused by the pulsation of a single star and not the interactions of a binary system to which earlier scholarship adhered. The type of pulsation was not determined until the publication of Eddington's 1917 paper 'The pulsation theory of Cepheid variables' which suggested that Cepheid variable stars were radial pulsators, a theory which has since been confirmed.

Cepheids are intrinsic variables that pulsate through radial expansion and contraction of the whole star. The pulsations of Cepheid stars are seen as periodic variations in the light output of the star over time. Cepheid stars tend to pulsate with periods of approximately 1 to 50 days, however, some pulsate with periods of over 100 days. The lightcurves of short period Cepheid variables $(\mathrm{P}<40$ days) have a distinctive asymmetrical shape, with a sharp increase in brightness followed by a long slow decrease in brightness; the distinctive shape of the short period Cepheid lightcurves change toward a sinusoidal shape for the longer period variables. A small number of Cepheid variable stars have shown bumps in the distinctive asymmetrical shape of the lightcurve. Cepheid variables that have a period of approximately 10 days display a bump near the maximum; at this period the lightcurve appears to have a double peak. The bump is found on the decreasing slope of the lightcurve for Cepheid variables with periods from approximately 7 to 10 days and on the increasing slope for periods greater than 10 days, and is due to resonance between

\footnotetext{
${ }^{5}$ Long period variables were given the file name long_period<Star_number>.dat, Cepheid variables were given the file name cepheid<Star_number $>$.dat and eclipsing binaries were given the file name eclipsing $<$ Star_number $>$.dat
} 
the fundamental pulsation mode and the second overtone. Cepheid variables with extremely long or short periods appear to have no bump and display smooth continuous variation.

In 1912 the American astronomer Henrietta Leavitt discovered that the apparent luminosity of SMC Cepheid variables was directly proportional to the logarithm of the period $(\log P)$. The apparent luminosity of these variables could be related directly to the absolute luminosity because the stars investigated by Henrietta Leavitt were all contained within the SMC and were, therefore, approximately the same distance from the Earth. Equation 3.2 shows that the apparent luminosity of a star as seen from Earth is inversely proportional to the square of the distance to the star.

$$
L_{a p}=\frac{L}{4 \pi d^{2}}
$$

Where $L_{a p}$ is the apparent luminosity, $L$ is the absolute luminosity and $\mathrm{d}$ is the distance between the observer and the star. Because of the relatively constant distance the $d^{2}$ in equation 3.2 becomes a constant value and the apparent luminosity becomes directly proportional to the absolute luminosity. This relationship together with the observed proportionality between the apparent luminosity and the logarithm of the period of Cepheid variables, allowed the period to be related to the absolute luminosity. This is known as the period-luminosity relationship for Cepheid variables. From the measured period of a Cepheid star, the period-luminosity relationship provides the absolute luminosity of the star. The distance to the star can be determined when equation 3.2 is applied to the apparent luminosity measurement and the absolute luminosity calculation. Prior to the discovery of the period-luminosity relationship, the only method available for measuring stellar distances was the parallax method, which observed the motion of close stars against distant background stars, caused by the motion of the Earth around the Sun.

Cepheid variables have large absolute magnitudes ranging from -2 to -6 . Because of the brightness of these stars Edwin Hubble, in 1923, was able to observe Cepheids in the Andromeda galaxy (M31) and calculate the first extra galactic distance based on the observations. Cepheid variable stars occupy a region of stellar evolution where stars are moving from the Red Giant phase along the instability strip, on the Hertzsprung-Russell diagram. The instability manifests itself as exploding nova, supernova or pulsation. 


\subsubsection{The search for Cepheid variables}

The search for Cepheid variables in the MOA database was conducted using a combination of the StarBase database, the $\mathrm{C}++$ extraction program, the Welch and Stetson variability index and the Schwarzenberg-Czerny period folding program. Standard deviations of the brightness and the Welch and Stetson variability indices were calculated by functions in the extraction program and used to remove stars with low levels of variability. Stars which were not excluded by the extraction program were then passed through the Schwarzenberg-Czerny period folding program which analysed the lightcurves for possible periodic signals. Lightcurves which were flagged by this program were examined after the program had searched all the stars recorded in the database. These lightcurves were confirmed as Cepheid variables by locating the position of each star on the field's colour magnitude diagram.

Section 3.2 describes the bump found in some Cepheid variable lightcurves. The position of the bump on the lightcurve is determined by the period of pulsation of the Cepheid variable and occurs on the downward slope of the lightcurve for Cepheids with periods less than approximately 10 days (see figures 3.7, 3.8 and 3.9). Cepheid variables with pulsation periods of approximately 10 days produce lightcurves with double peaks (see figure 3.6). Furthermore, Cepheids with a period over 10 days have bumps which occur on the upward slope of the lightcurve (see figure 3.10). Eighty-three Cepheid variables with periods ranging from 71 days to 21 hours were found in the MOA nlmc 1 fields. A catalogue of the all the LMC Cepheid Variables is shown in the table C.1. No attempt has been made to relate these variables to existing databases of LMC Cepheid variables.

The period luminosity relationship for Cepheid variables (described in section 3.2) allows the distance to Cepheid variables to be calculated from the period of pulsation. The search for variable stars in the MOA nlmc 1 field detected 83 Cepheids located in the LMC, a satellite galaxy of our own Milky Way. All of the stars detected within the LMC were at approximately the same distance from the Earth, therefore, the plot of magnitude versus the natural log of the period reveals a straight line, which represents the period luminosity relationship for Cepheid variables (figure 3.4). A group of points which were approximately 1 magnitude brighter and running parallel to the main group of cepheid variables were excluded, while calculating the linear relationship in figure 3.4. This was because these stars represent the type II Cepheid variables which have a different period luminosity relationship to type I cepheids ${ }^{6}$. From the straight line, the

\footnotetext{
${ }^{6}$ This thesis refferres to type I cepheids variables as cepheid variables
} 


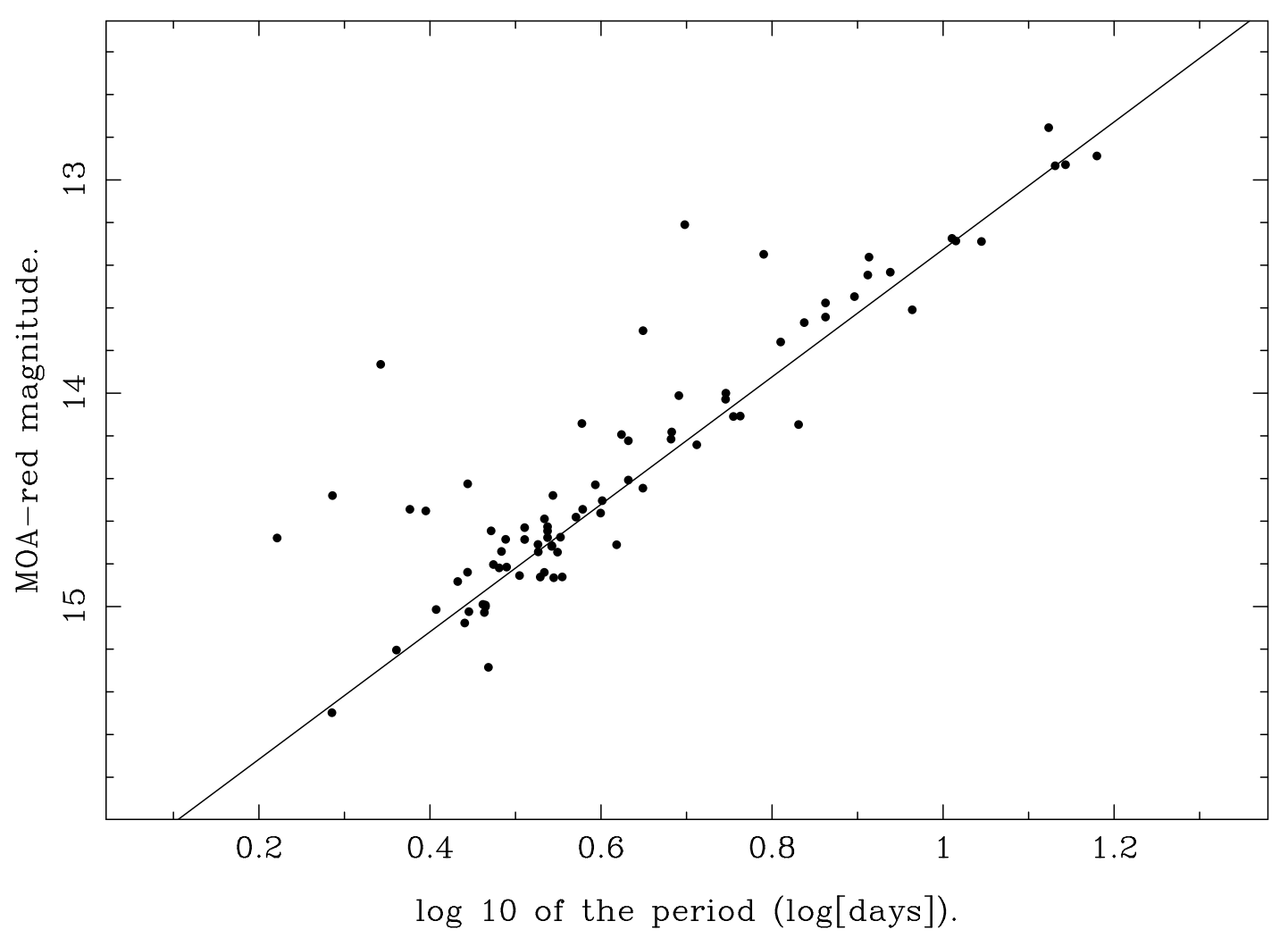

Figure 3.4: The figure shows the period luminosity relation for the Cepheid variables found in the MOA nlmc 1 star fields. The plot of magnitude versus the natural $\log$ of the period shows a straight line as expected for Cepheids located at the same distance from the observer.

relationship between the MOA-red magnitude and the period was calculated as

$$
\text { MOA-red }=(-3.0 \pm 0.5) \log _{10}[P]-(16.4 \pm 0.8)
$$

In 1997, the MOA nlmc 1 fields were observed using both MOA filters at ratios of $51 \%$ for MOA-red and $49 \%$ for MOA-blue, thereby allowing for the retrieval of colour information from the data; variations in the colour are related to changes in the surface temperature of the variable star. Figure 3.5 shows the period folded lightcurves of the Cepheid variable cepheid9436 observed through the MOA-red and MOA-blue filters. When the values of successive MOA-red and MOA-blue observations are subtracted, the difference can be plotted to show the colour variations over time. The wavelength of the radiation emitted from an object is directly related to the temperature of the object, hence changes in the colour of an object represent changes in the surface temperature of the object. 

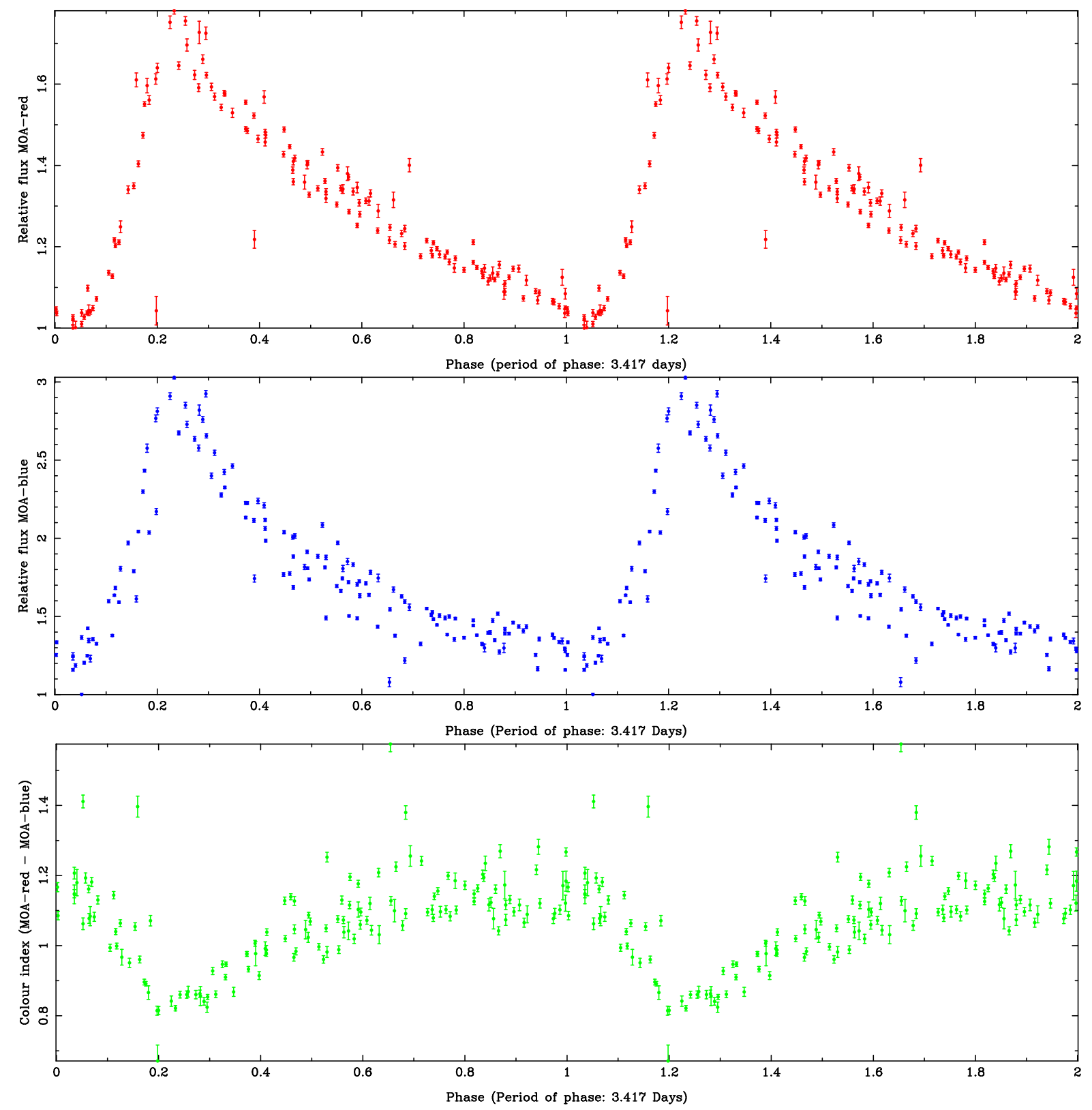

Figure 3.5: The MOA-red, MOA-blue and colour index plots of the Cepheid variable cepheid9436. 


\subsubsection{Lightcurves}

\section{The 1997 LMC data}
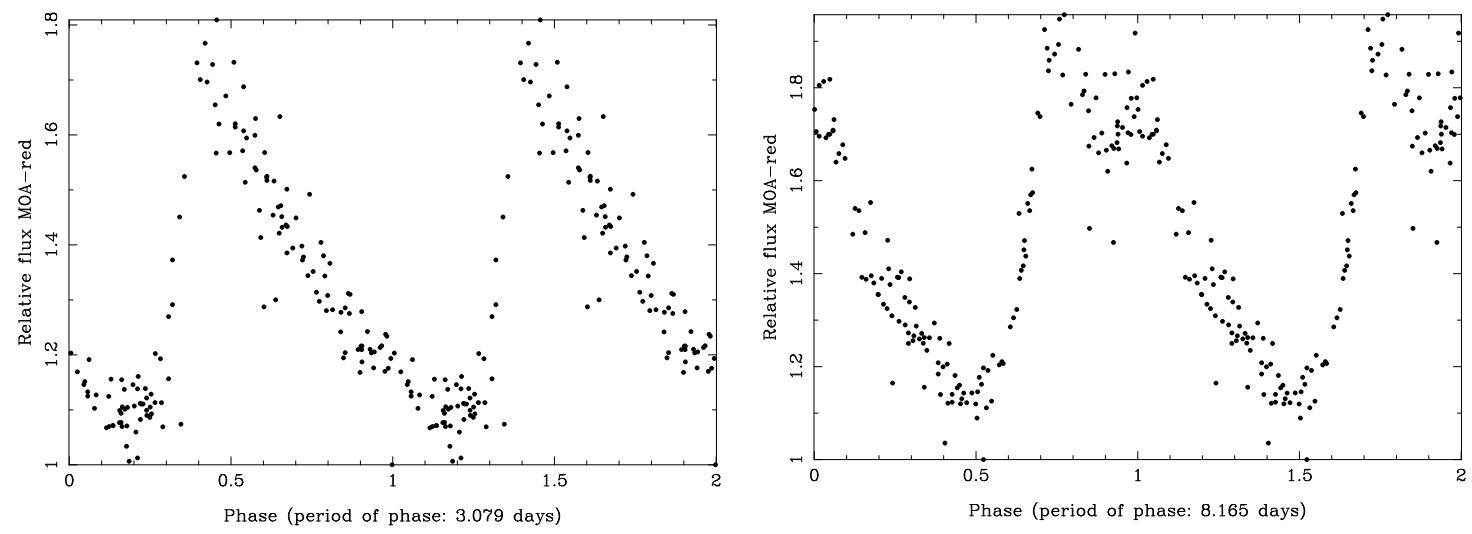

Figure 3.6: Periodogram of folded lightcurves cepheid1623 (left) and cepheid1731 (right).
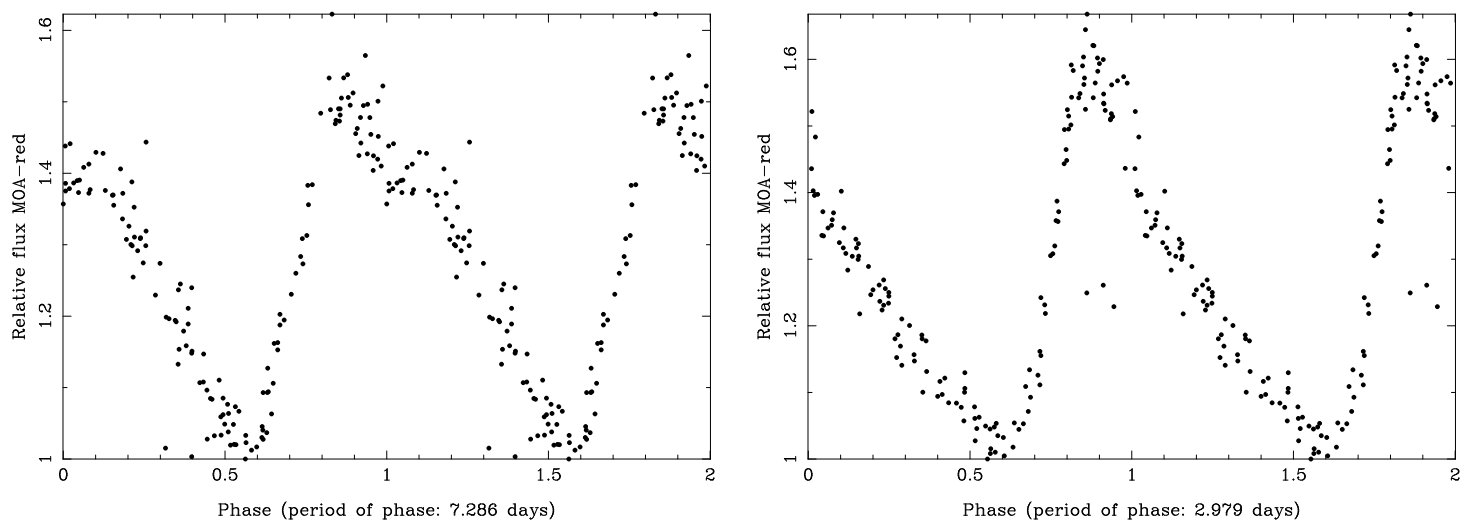

Figure 3.7: Periodogram of folded lightcurves cepheid1828 (left) and cepheid2308 (right).
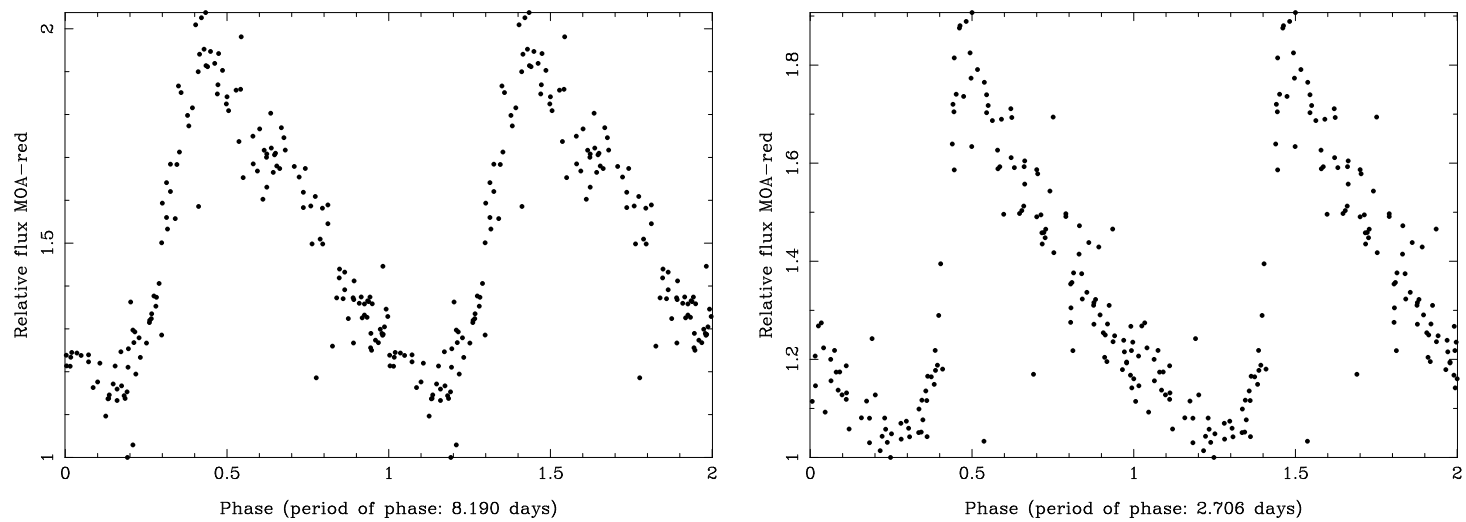

Figure 3.8: Periodogram of folded lightcurves cepheid3082 (left) and cepheid4604 (right). 

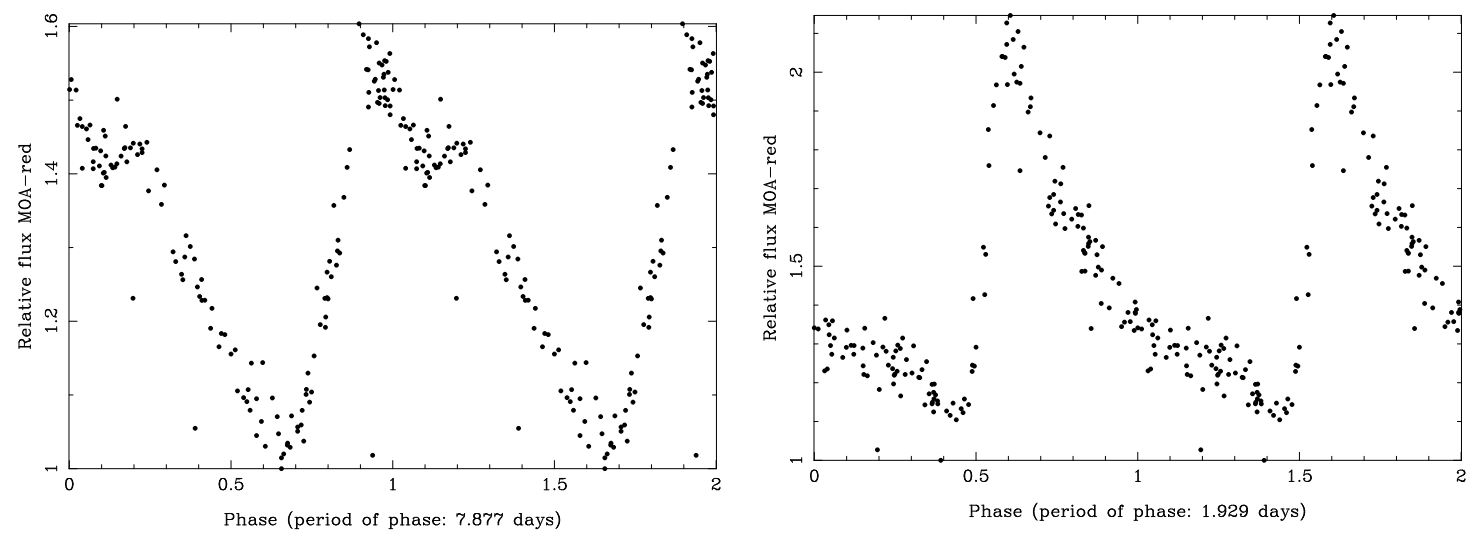

Figure 3.9: Periodogram of folded lightcurves cepheid4991 (left) and cepheid6142 (right).
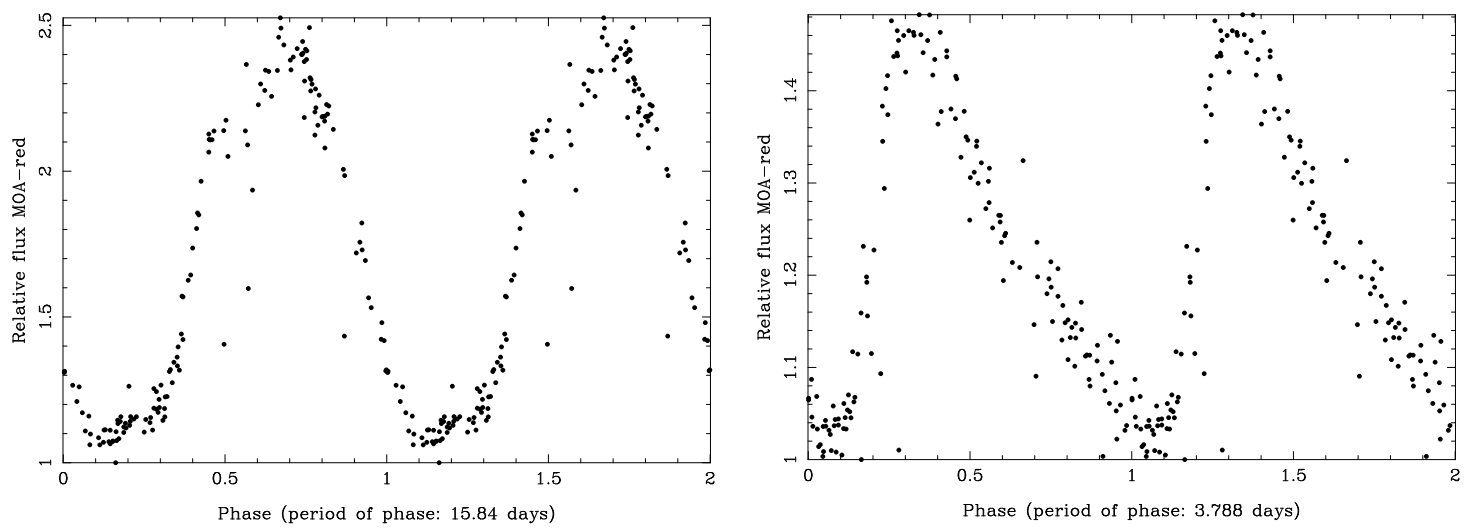

Figure 3.10: Periodogram of folded lightcurves cepheid8550 (left) and cepheid9642 (right).
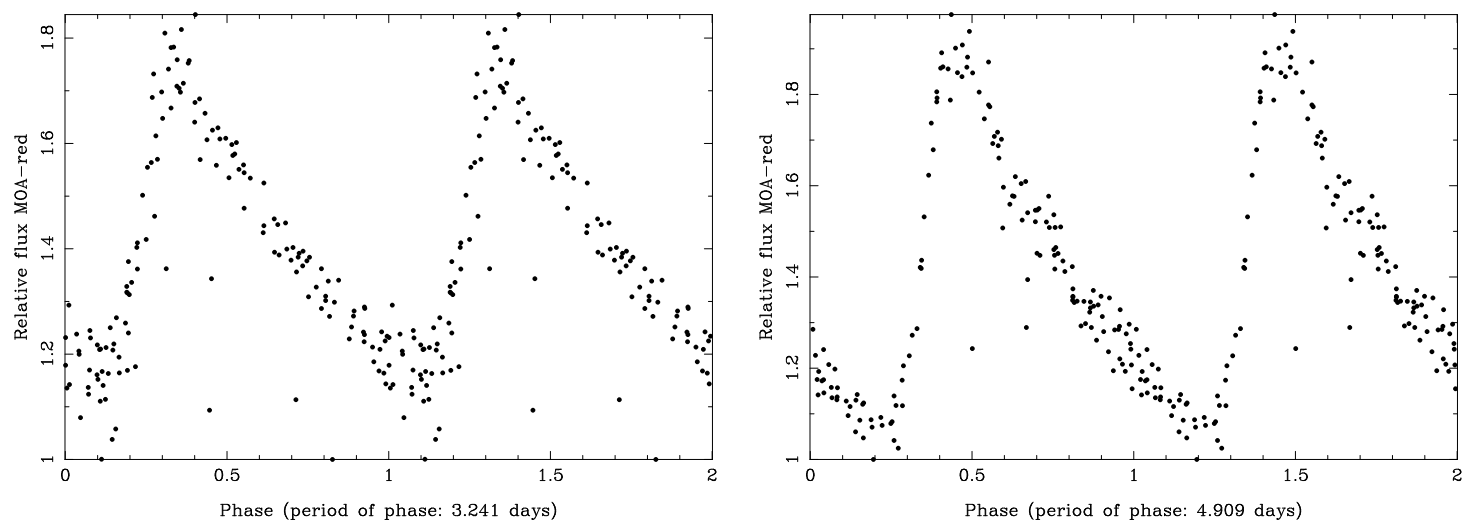

Figure 3.11: Periodogram of folded lightcurves cepheid10409 (left) and cepheid10966 (right). 


\subsection{Long period variables}

The long period variable class of stars are predominantly red giant stars, and includes semi-regulars, slow irregular variables, Mira variables and RV Tauri type variable stars. However, variations in the spectra and velocity curves of long period variables are complex and not thoroughly understood. Long period variable red giant stars belong to a critical, short-lived stage of stellar evolution of intermediate mass stars (the Asymptotic Giant Branch), which leads to planetary nebulae and white dwarfs.

Studies of long period variables have suggested that there is a period luminosity relationship similar to that of Cepheid variable stars (Glass \& Evans 1981) and hence they could be used as distance indicators. An advantage of using long period variables for distance calculation is the relative abundance of long period variables relative to Cepheid variables: long period variables occur five times more frequently than Cepheid variables. However, the relationship between period and luminosity for long period variables is not as well defined as the equivalent relationship for Cepheid variables.

\subsubsection{The search for long period variables}

Long period variable stars in the MOA LMC fields were found using the StarBase database, the $\mathrm{C}++$ extraction program, the Welch and Stetson variability index and the Schwarzenberg-Czerny period folding program (details are described in section 3.2.1). Colour magnitude diagrams, such as figure 3.3, were utilised to differentiate between Cepheid variables with long periods and long period variables with short periods. Lightcurves of long period variables were not folded into periodograms because the period of the pulsation was too large for the folding to produce useful plots. The search of the MOA LMC fields revealed 265 long period variable stars, a selection of these are shown in figures $3.13-3.18$.

RV Tauri variables are also incorporated in the long period variable class. These stars display semi-regular variations in brightness which alternate between deep and shallow minima with periods ranging from 30-150 days. The variations in the brightness are preceded by up to a quarter of the period by variations in the colour. Figure 3.12 shows the MOA-red and MOA-blue lightcurves of the variable star long_period4178. The dashed lines which appear at approximately 510 and 670 days represent local minima and maxima (respectively) for the MOA filter lightcurves. Variations in colour are also displayed in the figure 3.12, however, the dashed line associated with the local minima in the MOA filter lightcurves (day 510), coincides with a local maxima in the colour curve. This change in phase is also displayed at the local maxima located at day 670 , which is 

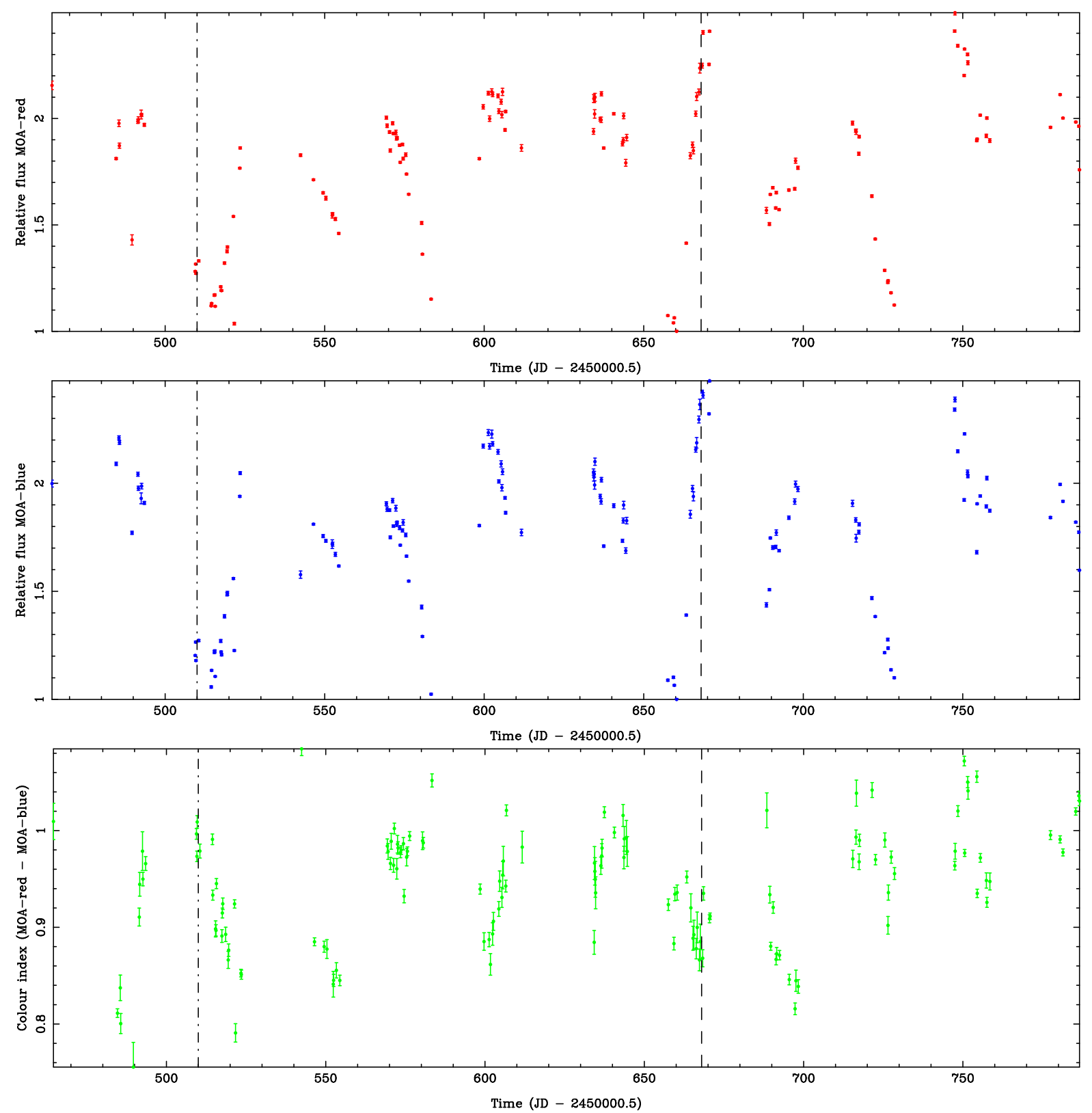

Figure 3.12: The MOA-red, MOA-blue and colour index plots of the RV Tauri variable long-period4178. The dashed lines located at approximately 510 and 670 days show the antiphase between the lightcurves and the colour curves. 
associated with a local minima in the colour curve. Although the colour curve shows a phase change, the degree of the change is difficult to determine because of the lack of clear alternating deep and shallow minima in the colour curve. The alternating deep and shallow minima found in the lightcurves of the variable long-period4178, together with the change in phase between the lightcurve and colour curve, suggests that the star is a RV Tauri type variable.

Figures 3.13-3.18 show a selection of lightcurves which contain long period variations in brightness. These lightcurves are consistent with the semi-regular, slow irregular or Mira variable category of star, but they will require observations over long time scales and spectral analysis in order to distinguish between the variable types. 


\subsubsection{Lightcurves}

\section{The 1997 LMC data}
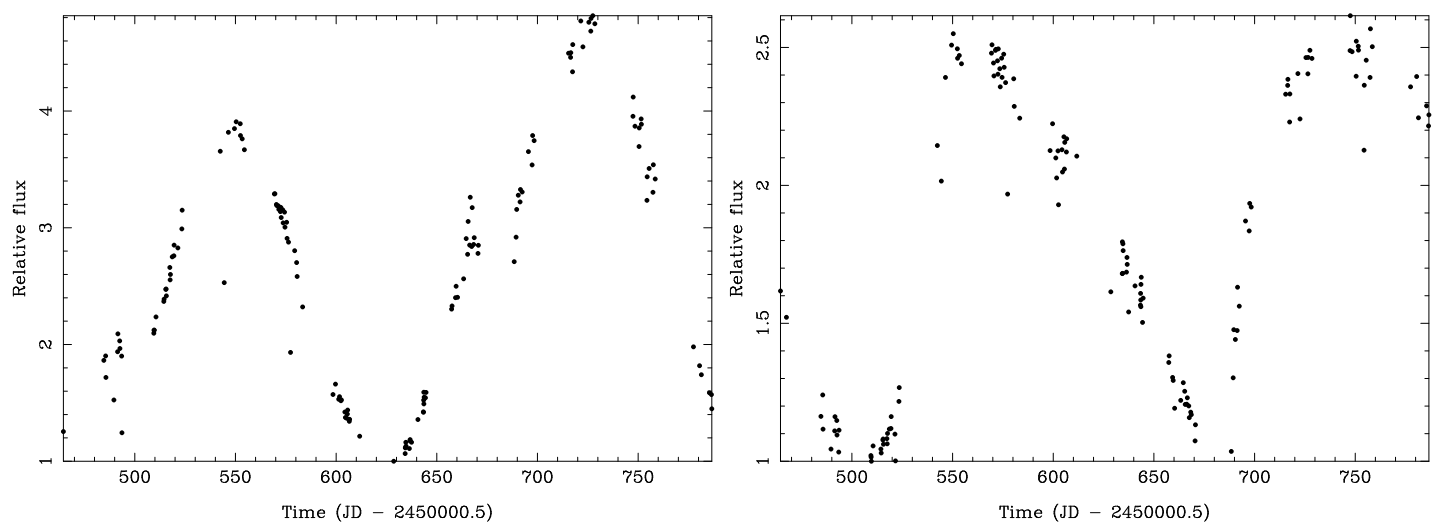

Figure 3.13: Lightcurves of the long period variables long_period2006 (left) and long_period3627 (right).
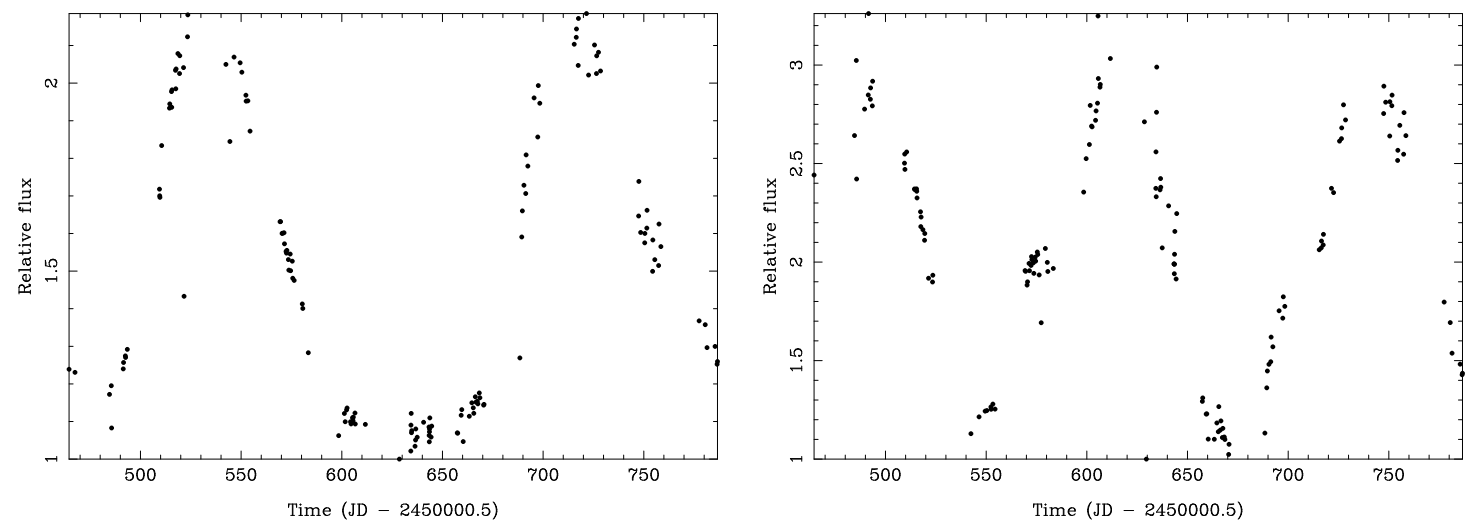

Figure 3.14: Lightcurves of the long period variables long_period4411 (left) and long_period6704 (right).
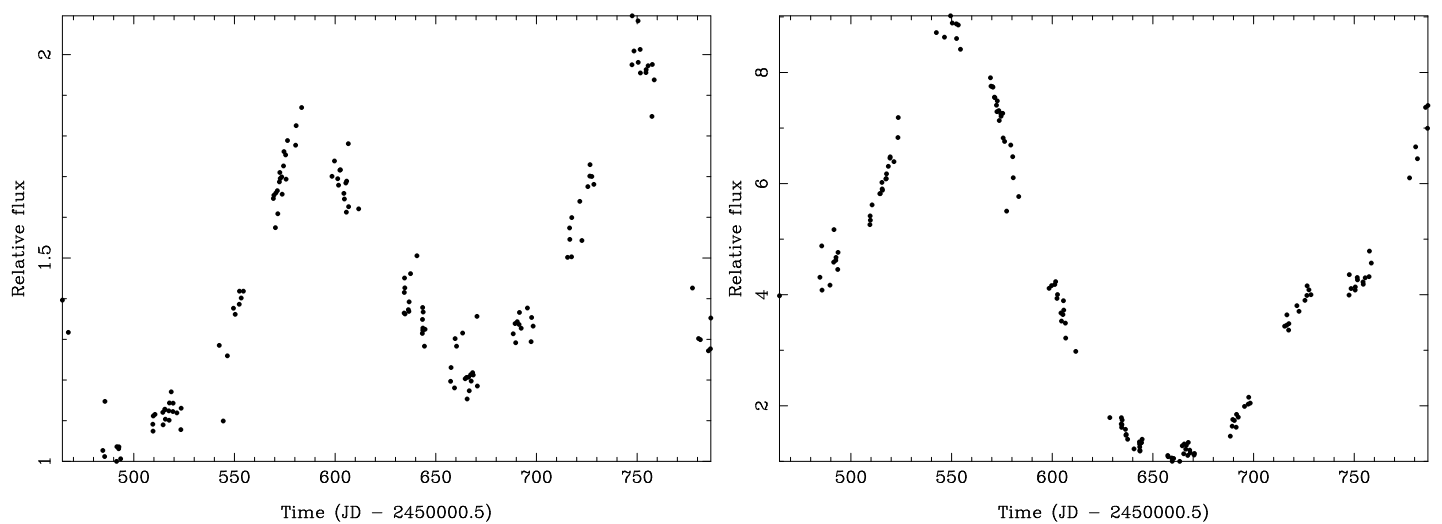

Figure 3.15: Lightcurves of the long period variables long-period10389 (left) and long_period10646 (right). 

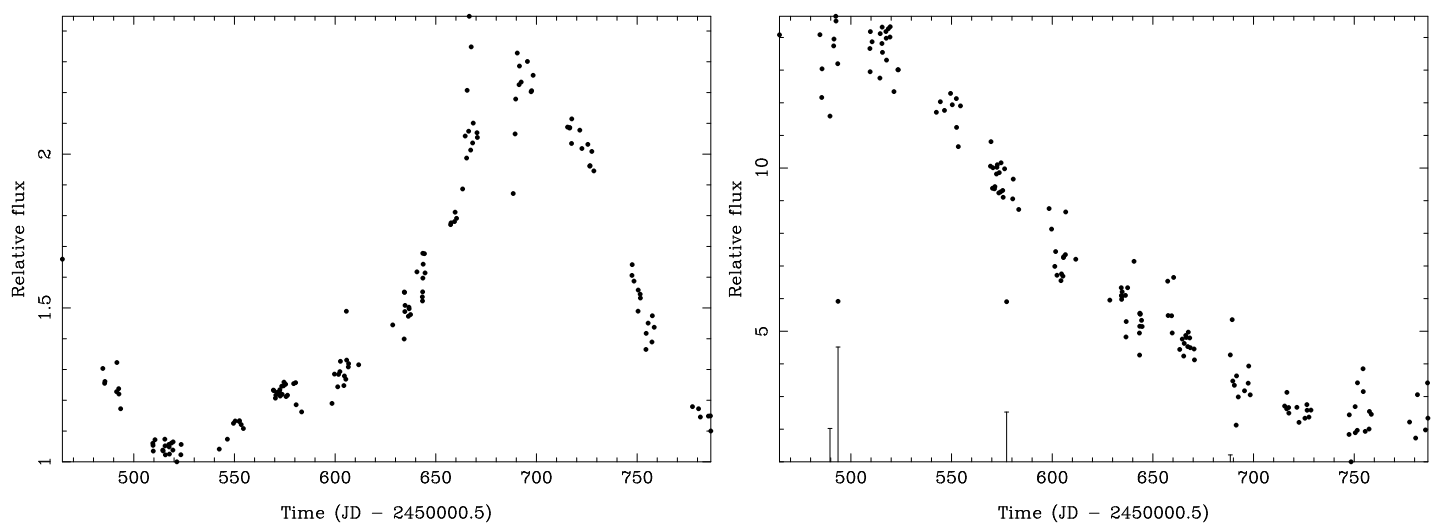

Figure 3.16: Lightcurves of the long period variables long-period10692 (left) and long-period10787 (right).
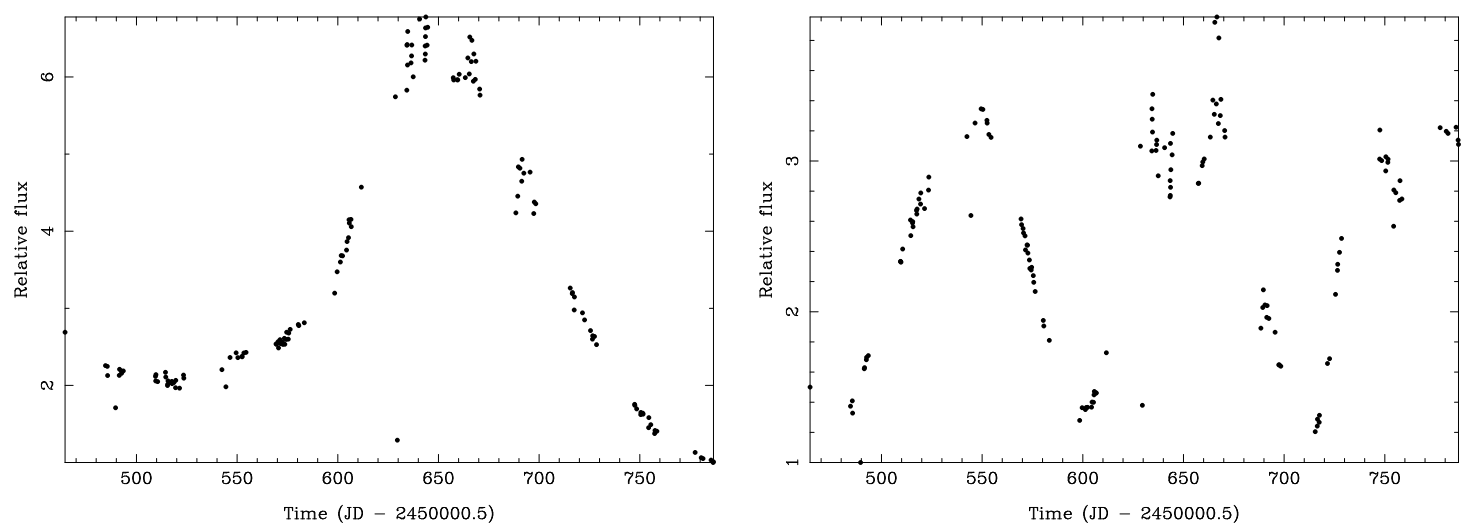

Figure 3.17: Lightcurves of the long period variables long-period10912 (left) and long-period11343 (right).
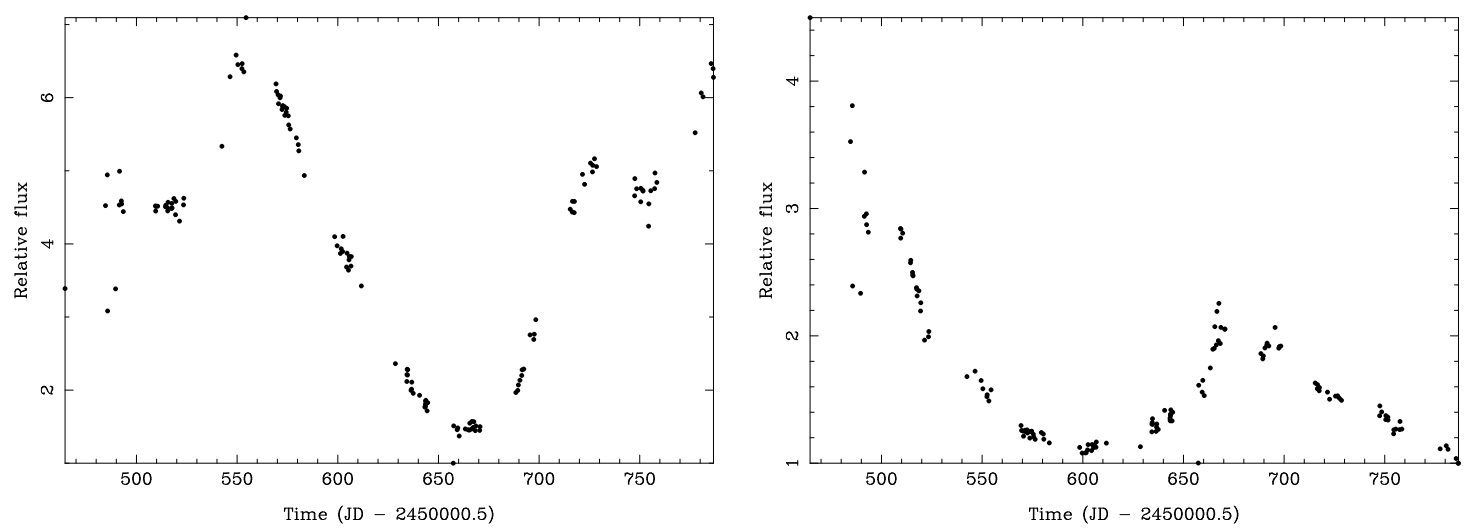

Figure 3.18: Lightcurves of the long period variables long-period19522 (left) and long-period 24952 (right). 


\subsection{Eclipsing binaries}

The first eclipsing binary system (Algol) was discovered in 1667 by the Italian astronomer Geminiano Montanari. It was not until 1782, however, that John Goodricke showed that the star varied with a period of 2.87 days. Goodricke suggested that this was caused by partial eclipses of the star by another body, a hypothesis that was proved to be correct by spectrographic analysis performed by the German astronomer H.C.Vogel, in 1889 .

Eclipsing binaries occur when a star in a binary system passes directly between the companion star and the observer on Earth, which causes the amount of light received from system to be reduced. Spectroscopic studies of the light from binary stars allows the ratio of masses for each of the components to be calculated, however, the sum of the masses can only be determined if the orbital inclination of the system relative to the Earth $(i)$ is known. Eclipsing binaries have an orbital inclination (relative to the Earth) of approximately $i=90^{\circ}$, therefore, the sum of the masses of the system can be calculated.

\subsubsection{The search for eclipsing binaries}

Two separate searches for eclipsing binaries were conducted in the 1997 MOA LMC and 2000 Galactic Bulge data. Eclipsing binaries in the LMC fields were discovered using the Schwarzenberg-Czerny period folding program, which was used to search for Cepheid and long period variables. This search method revealed four eclipsing binaries in the LMC fields. The second search looked through the 2000 MOA Galactic Bulge fields, and used a variation of the phase space search program (described in section 5.2), which was originally designed to search for planetary transits. This search program was used because eclipsing binaries produce lightcurves which are similar to those produced by planetary transits with larger variations in brightness. Because of these similarities, the search for eclipsing binaries in the Galactic Bulge fields was used to test the phase space search program on real MOA data.

The phase space search program used a square well model curve (shown in equation 5.2) for a coarse search over a large range of trial orbital periods. This square well had a depth of $2 \%$ of the mean brightness level and a width of $4 \%$ of the trial period while searching for planetary transits. These dimensions were altered to a depth of $15 \%$ of the mean brightness level and a width of $10 \%$ of the trial period during the search for eclipsing binaries. Nineteen eclipsing binaries were found in the 2000 MOA Galactic Bulge fields and are catalogued in appendix C.3 (a selection of lightcurves are displayed in 
figures 3.26-3.31), these lightcurves have been labelled with the "_a" string to differentiate them from the eclipsing binaries found in the LMC fields.

The search of the Galactic Bulge fields revealed nineteen eclipsing binaries from a search of approximately 40000 stars (0.05\%), a figure significantly higher than the 4 eclipsing binaries that were previously found in the LMC fields from a search of approximately 300000 stars $(0.001 \%)$. These results indicate that the combination of the $\mathrm{C}++$ extraction program and the Schwarzenberg-Czerny period folding program have difficulty in locating eclipsing binaries. Consequently, a second search of the LMC fields was conducted using the phase space search program, which was used on the Galactic Bulge data. This search revealed 40 eclipsing binaries $(0.01 \%)$, and included the four previously discovered with the Schwarzenberg-Czerny period folding program. Although this was a significantly larger number in comparison to the previous search of the LMC fields, the percentage of eclipsing binaries was a factor of five smaller than percentage found in the Galactic Bulge fields. A catalogue of the the LMC eclipsing binaries is shown in appendix C.2 (a selection of lightcurves are shown in figures 3.20-3.25).

Figure 3.19 shows the colour variation of the eclipsing binary eclipsing2378. Eclipsing binaries are usually classified into three groups: the Algol type (EA), the $\beta$ Lyrae type (EB) and the W UMa type (EW); they are classified by colour variations and the shape of the lightcurve. Colour changes can be used to estimate the spectral type of the stars, however, the shape of the lightcurve is the primary means of identifying the type of eclipsing binary. Algol type lightcurves are relatively constant between eclipses and do not display variations in brightness due to ellipticity ${ }^{7}$ and reflection effects. ${ }^{8}$ Lightcurve eclipsing25968_a, shown in Figure 3.31, is an example of the Algol type eclipsing binary. Eclipsing binary types $\beta$ Lyrae (EB) and W UMa (EW) both display continuous variations in brightness between eclipses. However, EB types show a significant difference between primary and secondary eclipses, while the difference in EW types is relatively small. Moreover, the orbital periods of EB types are generally greater than one day, while the EW types display periods generally less than one day. Lightcurve eclipsing8138 (figure 3.24) is an example of an EB type eclipsing binary, while eclipsing2476_a (figure 3.28 )is an example of EW type.

\footnotetext{
${ }^{7}$ The ellipticity effect occurs when a star is stretched into an ellipsoid shape by the gravity of its binary companion. This ellipsoid shaped object will show varying amounts of surface area (and therefore brightness) as it rotates about the centre of mass of the binary system.

${ }^{8}$ The reflection effect occurs when the close proximity of a binary system heats the area on a star closest to its binary companion. This heated area is brighter than the rest of the star and causes variations in brightness as the star rotates about the centre of mass of the binary system.
} 

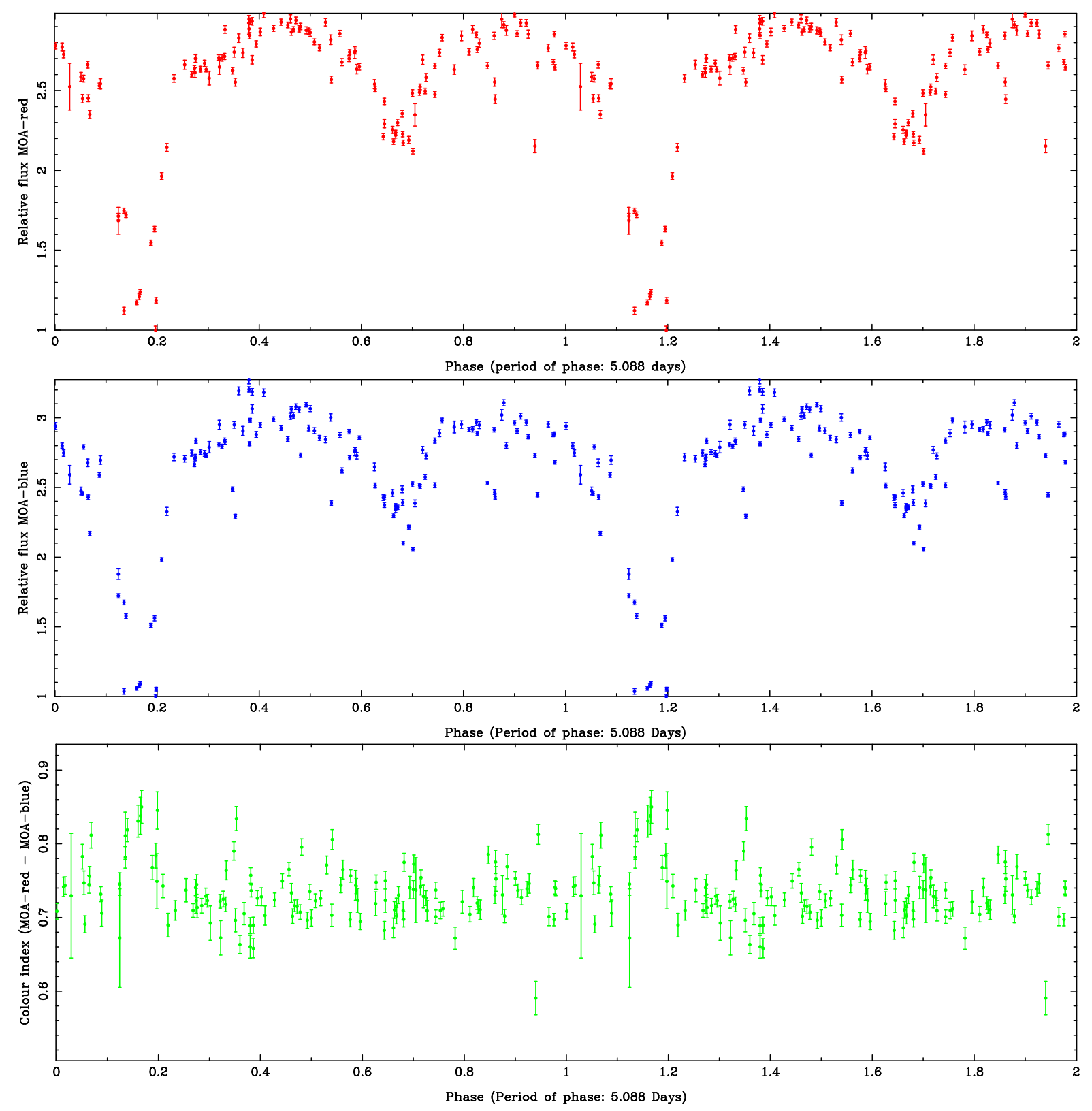

Figure 3.19: The MOA-red, MOA-blue and colour index plots of the eclipsing binary eclipsing5746. 


\subsubsection{Lightcurves}

The 1997 LMC data
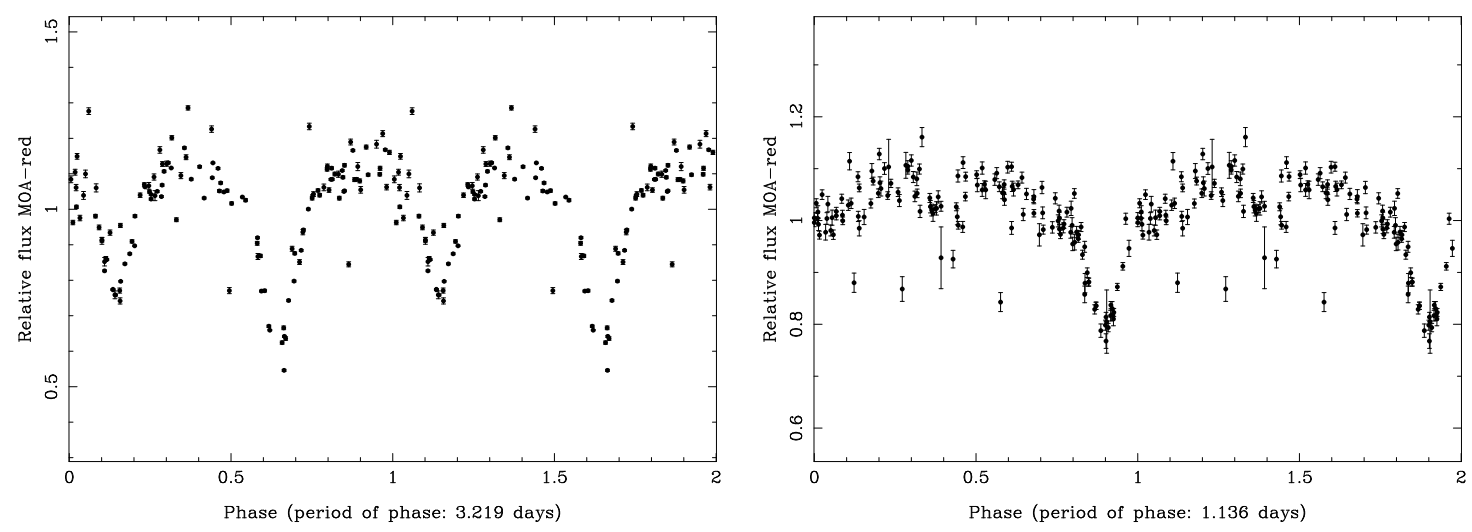

Figure 3.20: Periodogram of folded lightcurves eclipsing173 (left) and eclipsing2138 (right).
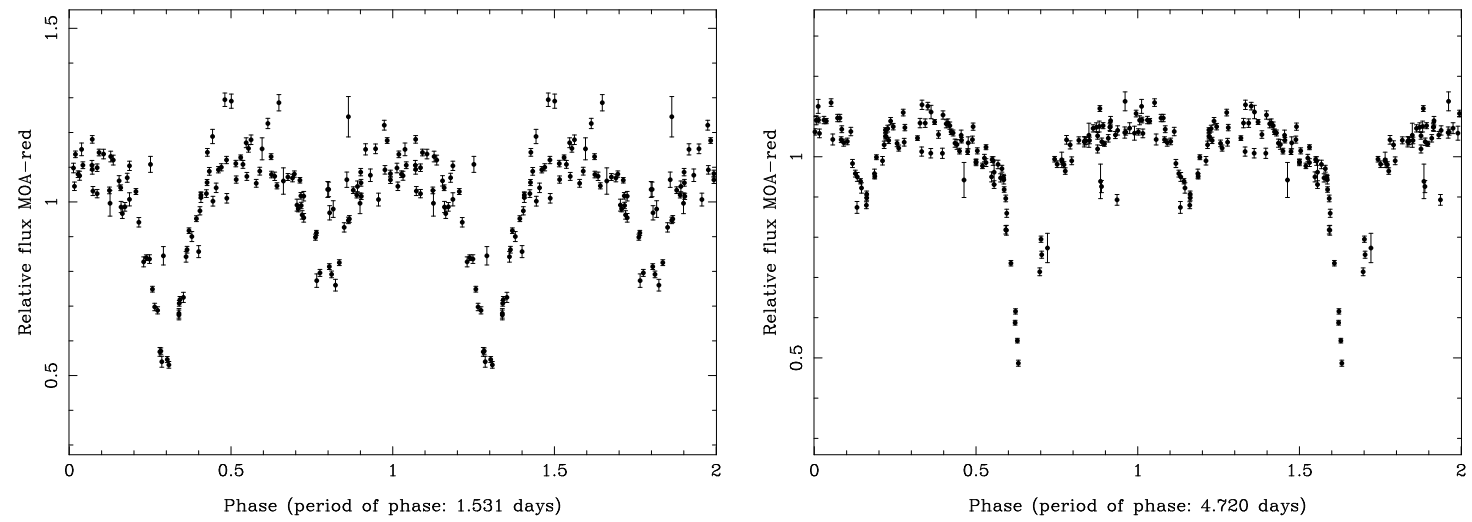

Figure 3.21: Periodogram of folded lightcurves eclipsing2301 (left) and eclipsing2654 (right).
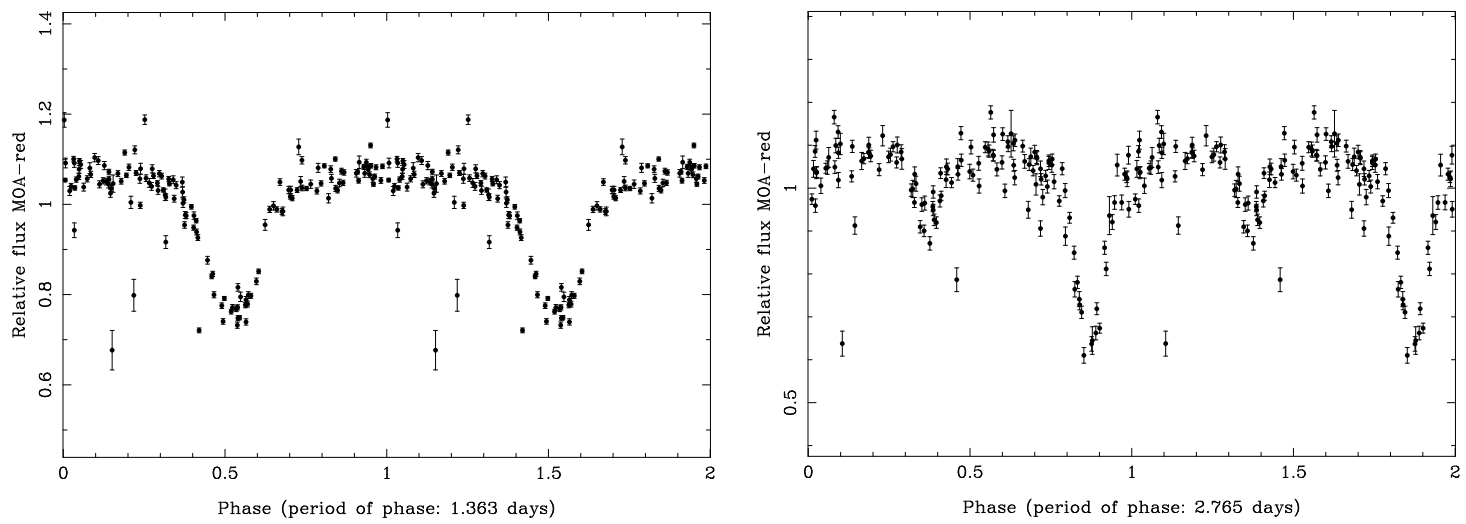

Figure 3.22: Periodogram of folded lightcurves eclipsing2819 (left) and eclipsing4811 (right). 

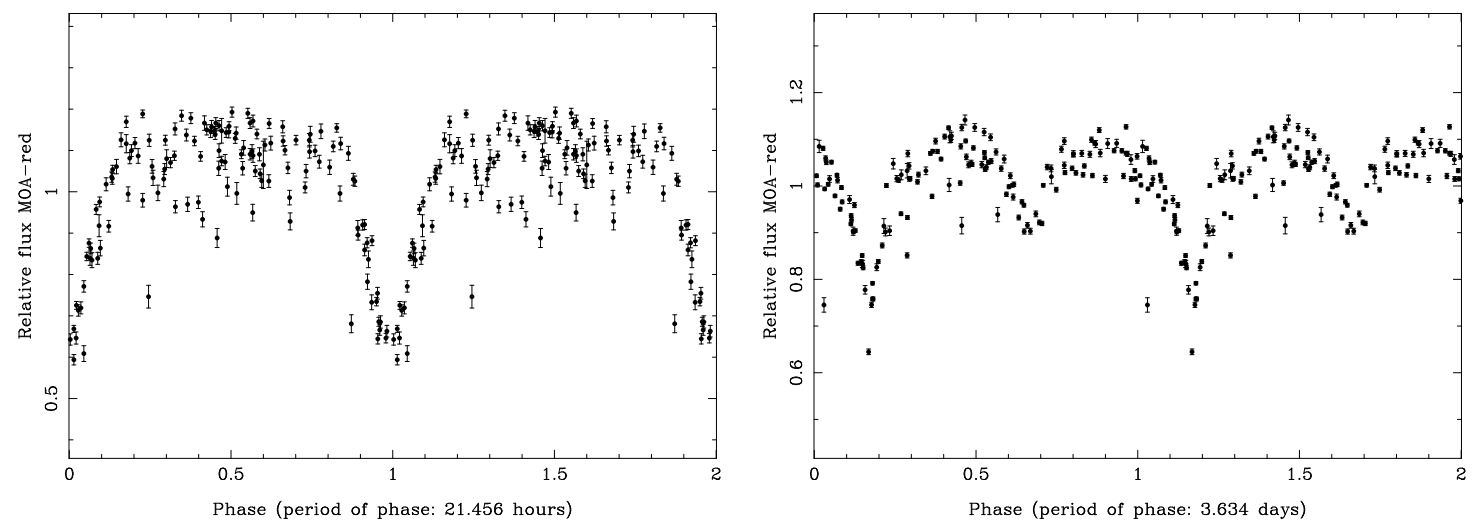

Figure 3.23: Periodogram of folded lightcurves eclipsing5078 (left) and eclipsing5462 (right).
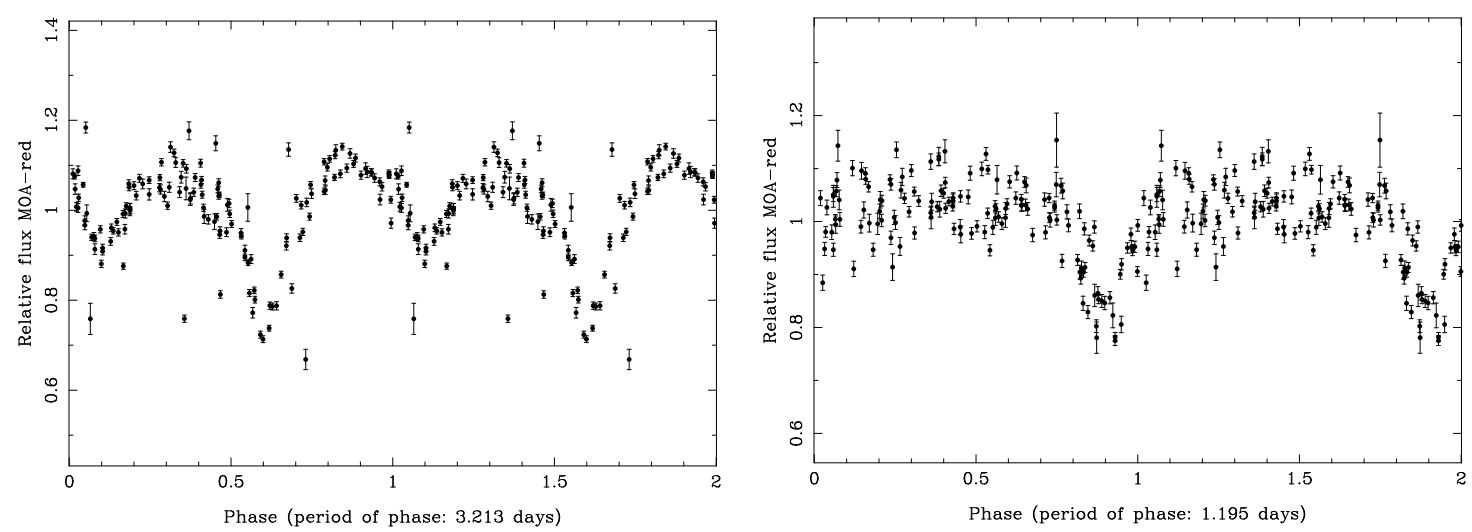

Figure 3.24: Periodogram of folded lightcurves eclipsing8138 (left) and eclipsing9411 (right).
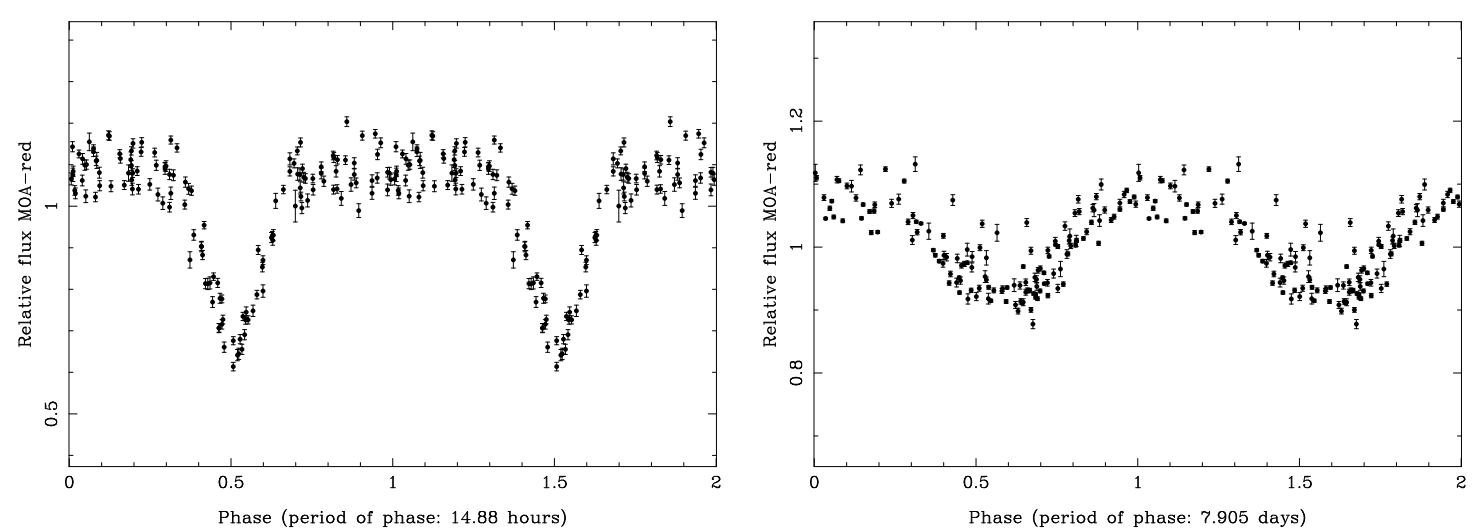

Figure 3.25: Periodogram of folded lightcurves eclipsing10089 (left) and eclipsing11441 (right). 
The 2000 Galactic Bulge data
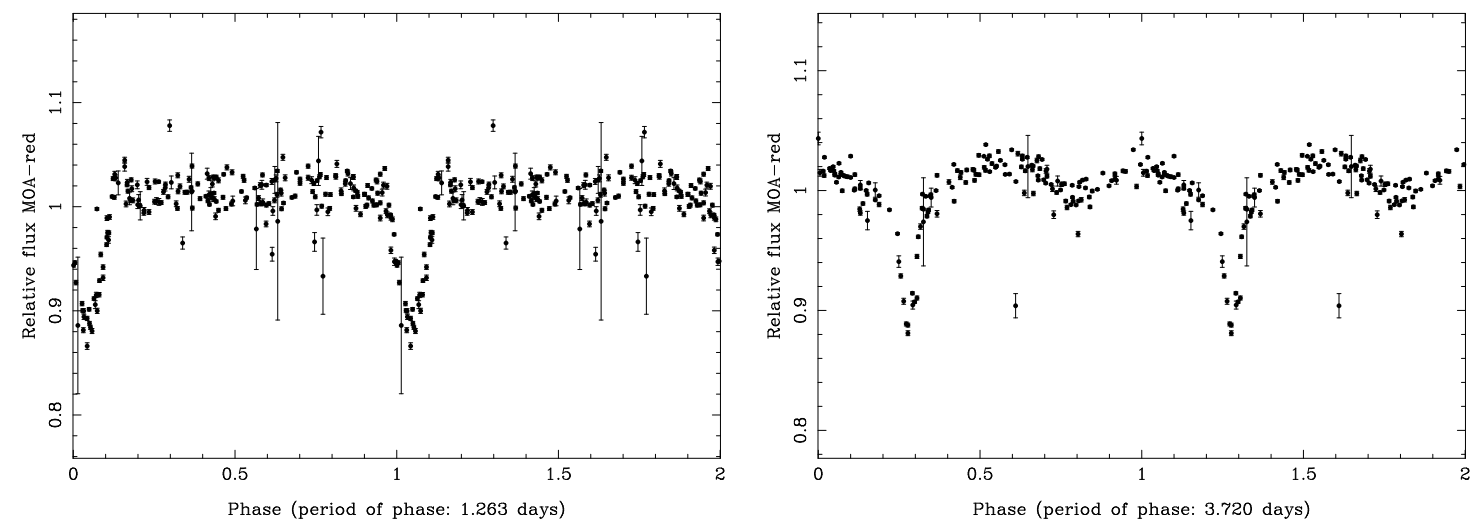

Figure 3.26: Periodogram of folded lightcurves eclipsing486_a (left) and eclipsing784_a (right).
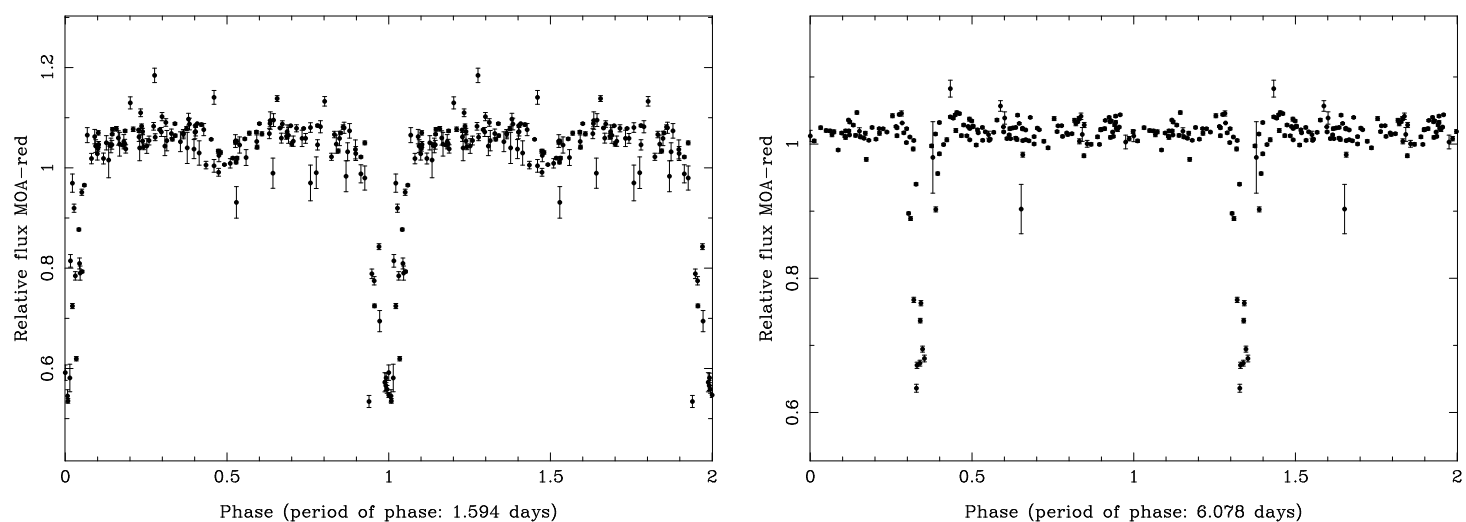

Figure 3.27: Periodogram of folded lightcurves eclipsing1010_a (left) and eclipsing2143_a (right).
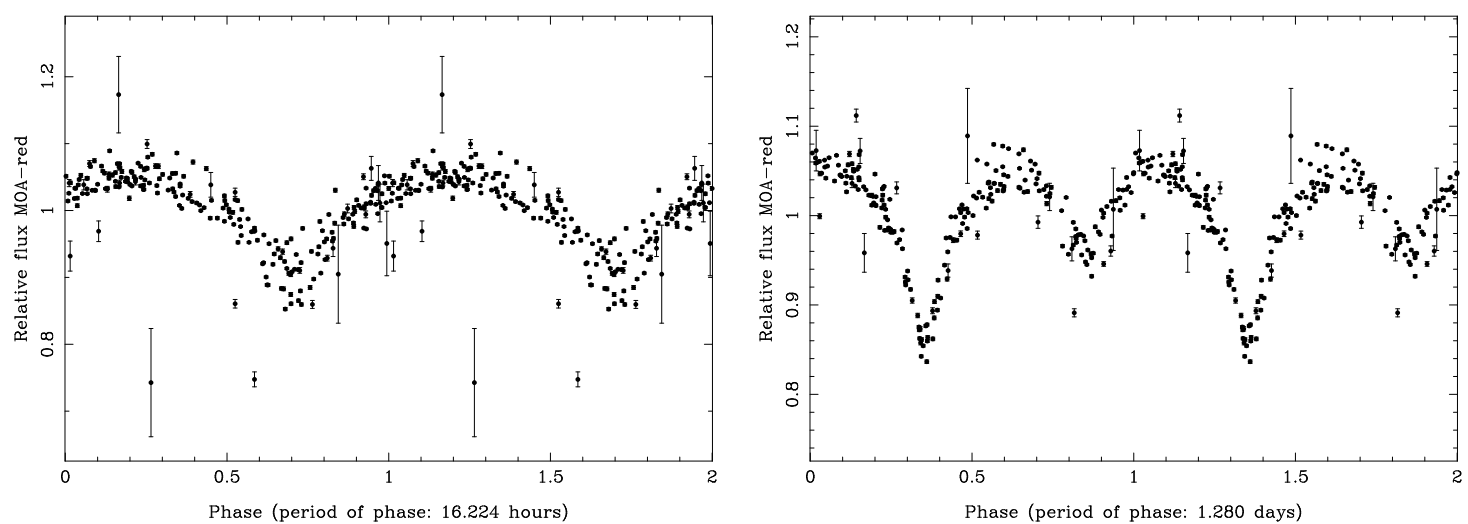

Figure 3.28: Periodogram of folded lightcurves eclipsing2476_a (left) and eclipsing5174_a (right). 

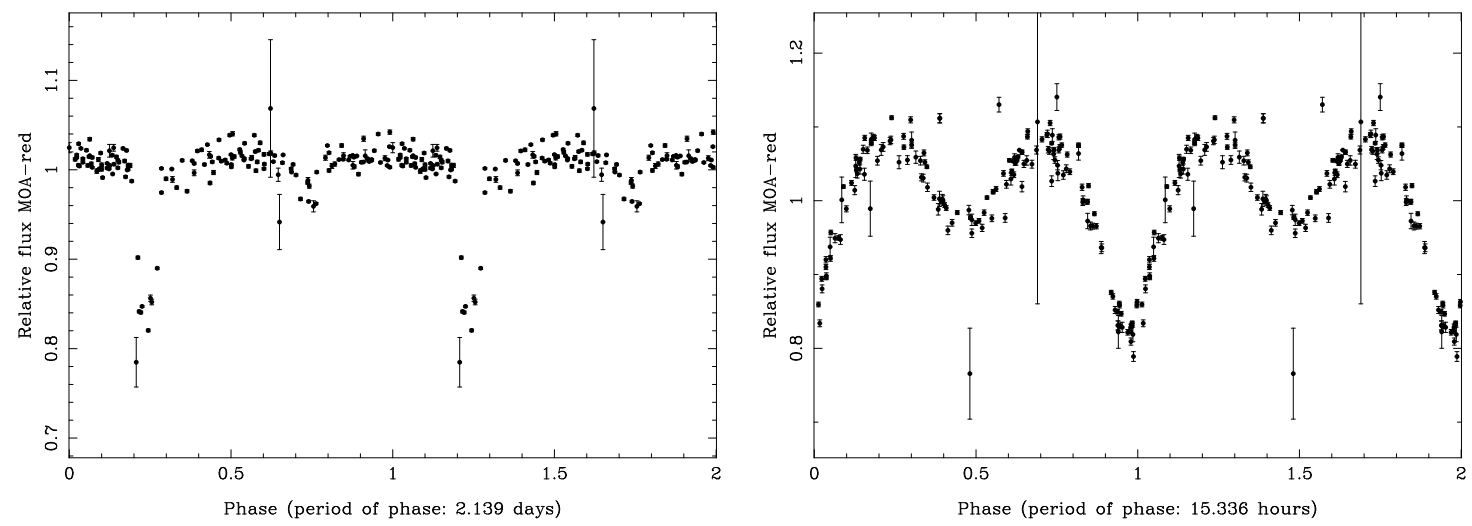

Figure 3.29: Periodogram of folded lightcurves eclipsing5286_a (left) and eclipsing5441_a (right).
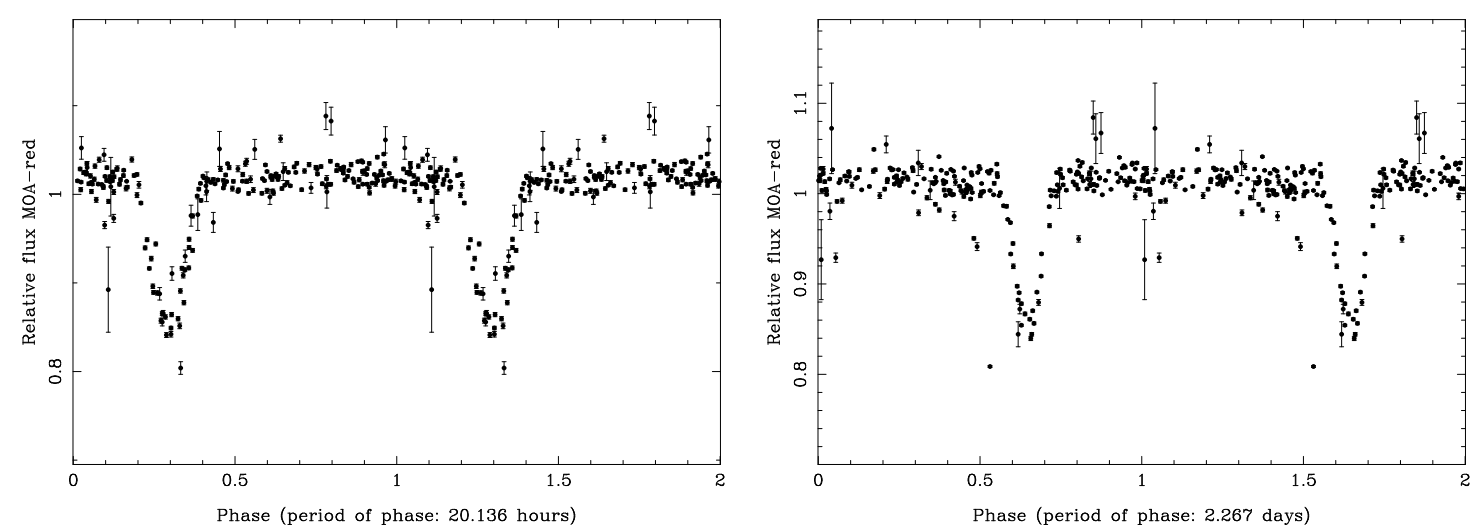

Figure 3.30: Periodogram of folded lightcurves eclipsing13327_a (left) and eclipsing16391_a (right).
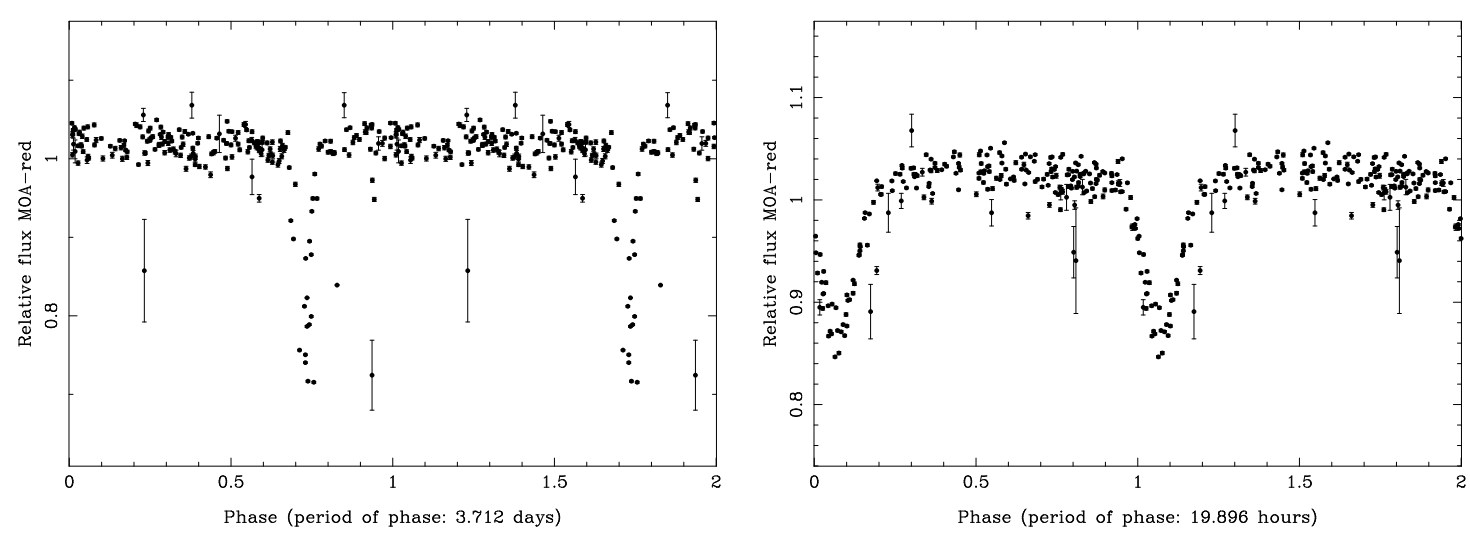

Figure 3.31: Periodogram of folded lightcurves eclipsing25968_a (left) and eclipsing26988_a (right). 


\section{Chapter 4}

\section{Gravitational microlensing}

\subsection{History of gravitational lensing}

The first written account of the deflection of light by gravity, 'On the deflection of a light ray from its straight motion due to the attraction of a world body which it passes closely', was published in 1804 by Johann Soldner, a German geodesist, mathematician and astronomer working at the Berlin Observatory. Solder explored the deflection of light by gravity within a Newtonian mechanics framework, and determined that light close to the solar limb would be deflected by an angle of 0.84 ". In 1911, Albert Einstein investigated the influence of gravity on light, while incorporating gravitational effects into his Special Theory of Relativity, and calculated the same value for the deflection of light by the Sun as Solder had one hundred years earlier. The original deflection value calculated by Einstein in 1911 used Special Relativity (Einstein 1911). After the complete development of the General Theory of Relativity (Einstein 1916), Einstein revisited the deflection of light by the mass of the Sun and calculated a new value for the angle of deflection which was double the original value. Experimental evidence of this deflection value was determined to within $20 \%$ during the 1919 solar eclipse, which was observed by a group led by English astronomer Arthur Eddington. The observation of the deflection angle was the second observational confirmation of Einstein's General Theory of Relativity (the first being the correct prediction of Mercury's perihelion motion)

The deflection of light by massive objects is now referred to as gravitational lensing. The term lensing is used because divergent light rays can be deflected by massive objects toward the same point in space, similar to the way in which a glass lens refracts divergent light toward a common focal point. Gravitational lensing can produce multiple images of a single object. In 1924, the German physicist Orest Chwolson put forward the idea of a "fictitious double star" and the mirror-reversed nature of the secondary image. 
Influenced by Einstein, the Swiss astrophysicist Fritz Zwicky suggested in 1937 that galaxies were much more likely to be gravitationally lensed than stars and that the gravitational lens effect could be used as a 'natural telescope'. When quasi-stellar objects (quasars) were discovered (Schmidt 1963), American scientist Jeno Barnothy introduced them as a possible source for gravitational lensing (Barnothy 1965). In 1979, the first double quasar was discovered and was confirmed to be a real gravitational lens (Walsh, Carswell \& Weymann 1979 and Weymann et al. 1979).

\subsection{Gravitational microlensing surveys}

The Polish astrophysist Bohdan Paczyński first used the term 'gravitational microlensing' (Paczynski 1986a) to describe the gravitational lensing by objects in our galaxy of extra galactic source objects. The term was derived from the micro-arcsecond separation of images produced by these lensing events. Paczyński's paper, 'Gravitational microlensing by the galactic halo' (1986a), outlined an observational procedure for observing these microlensing events. The targets suggested by Paczyński were the extra galactic objects the Large Magellanic Cloud (LMC), the Small Magellanic Cloud (SMC), the Andromeda galaxy (M31) and the Triangulum galaxy (M33). ${ }^{1}$ Following the publication of Paczyński's paper, several groups were formed expressly for the purpose of observing galactic halo microlensing events.

The MACHO (Massive Compact Halo Objects) ${ }^{2}$ project was founded in 1992 and undertook observations from 1993 until 1999, at Mount Stromlo Observatory in Canberra, Australia. Comprising members of the Center for Particle Astrophysics at the University of California, and the Lawrence Livermore National Laboratory, California, USA, the collaboration carried out observations using eight 2048 by 2048 CCDs (Charged Couple Devices) which were mounted on the $1.27 \mathrm{~m}$ Great Melbourne Telescope, at Mount Stromlo. The MACHO collaboration was credited with the first detection of a microlensing event.

Presently carrying out its third phase of operation, the OGLE (Optical Gravitational Lensing Experiment) project began its first phase (OGLE I) in 1992, using the 1.3m Warsaw Telescope at the Las Campanas Observatory in Chile. The project is a collaboration between Carnegie Institution of Washington, Princeton University, USA and Warsaw

\footnotetext{
${ }^{1}$ The Galactic Bulge has since been added to the list of targets.

${ }^{2}$ The acronym MACHO was coined by Kim Griest (Griest 1991), and describes the dense objects contributing to halo dark matter.
} 
University, Poland. The third phase of operation (OGLE III) uses a 'second generation' CCD mosaic camera with eight thinned SITe 2048 by 4096 CCDs.

The EROS (Experience pour la Recherche d'Objets Sombres) project was founded in 1990 and comprises members from the College de France and the Laboratoire de l'Accélérateur Linéaire, France. EROS I used a 40cm telescope at La Silla, European Southern Observatory (ESO) with a 1024 by 1024 pixel CCD camera. EROS II began in 1995 using the $1.5 \mathrm{~m}$ telescope (the MARLY telescope) which had been moved from the Observatoire de Haute-Provence (OHP) observatory in France to ESO in Chile for the EROS II Observations. The MACHO, OGLE and EROS projects all represent groups that have focused exclusively on searching for microlensing events.

Several groups within the microlensing community also undertake follow-up observational work on microlensing events that have been previously detected by other groups. The MPS (Microlensing Planet Search) Project was established in 1997 to observe microlensing events with the $1.9 \mathrm{~m}$ telescope at the Mount Stromlo Observatory and the $1.5 \mathrm{~m}$ telescope at the Boyden Observatory in South Africa. MPS involves collaborators from the University of Notre Dame and the University of Washington, USA, Mount Stromlo and Monash University, Australia and utilises microlensing alerts from the EROS and OGLE groups. The MPS group also utilised MACHO project microlensing alerts while the MACHO project was operational.

The PLANET (Probing Lensing Anomalies NETwork) project began in 1995 with access to the Dutch/ESO 0.9m telescope at La Silla, Chile, the South African Astronomical Observatory (SAAO) 1.0m telescope in Sutherland, South Africa, and the Perth $0.6 \mathrm{~m}$ and Hobart 1.0m Australian telescopes. More recently, the Yale $1.0 \mathrm{~m}$ at Cerro Tololo Inter-American Observatory (CTIO), Chile has taken over from the Dutch/ESO 0.9m. The PLANET project members comprise scientists from the Kapteyn Institute in the Netherlands, Space Telescope Science Institute in America and South African Astronomical Observatory in South Africa. Microlensing alerts from the OGLE and EROS groups were used by the PLANET project, and MACHO alerts were also utilised by the project, while the MACHO project was active. 


\subsection{Applications of microlensing surveys}

\subsubsection{Dark matter}

The primary objective of the first microlensing projects was to search for evidence of dark matter. The presence of dark matter was first inferred in 1932 by the physicist and astronomer Jan Oort, who studied the motion of stars above the disk of the Milky Way. By observing the motion of the stars, Oort was able to calculate the strength of the gravitational force required to maintain the star's orbit about the galactic center and he used these calculations to establish the approximate mass of the galaxy. However, the amount of mass calculated did not agree with amount of visible mass detected; the mass calculated was approximately a factor of 2 greater than the visible mass. Therefore, it was assumed that a form of non-visible mass or dark matter was present in order to account for the difference.

The positions and velocities of galactic halo objects were calculated by observing both the motion of spiral arms in distant galaxies and the motion of orbiting gas clouds in the the Milky Way's galactic halo. Results have indicated that the velocity of objects at large distances from galactic centres do not decrease with increasing distance as was previously hypothesized. Indeed, the motion observed suggests that the mass of the galactic halo continues to increase with increasing distance. As a result of these calculations it is believed that an increasing amount of dark matter must exist in order to account for the increasing mass of galactic halos.

Two principal types of dark matter have been postulated: Baryonic and Non-Baryonic. Baryonic dark matter comprises objects that have condensed from a cloud of gas to form massive dense objects and range from the smallest scale large gas clouds and brown dwarf stars ${ }^{3}$ to large scale Very Massive Objects (VMO) ${ }^{4}$. Non-Baryonic Matter comprises individual 'exotic particles' with a small but finite mass and include the massive neutrinos and theoretical particles which have been labelled Weakly Interactive Massive Particles (WIMPs). Neutrinos are weakly interacting, electrically neutral particles involved in nuclear interactions. Because they are so numerous, the discovery of a small neutrino mass would contribute considerably to the dark matter mass.

In 1986, Paczyński suggested that:

\footnotetext{
${ }^{3}$ Brown dwarf stars are condensed objects that are not massive enough to create the temperatures or pressures needed to begin nuclear fusion.

${ }^{4} \mathrm{VMO}$ are black holes that have condensed from objects greater than approximately $300 M_{\odot}$
} 
Monitoring the brightness of a few million stars in the Magellanic Clouds over a time scale between $2 \mathrm{hr}$ and 2 yr may lead to a discovery of "Dark Halo" objects in the mass range $10^{-6}-10^{2} M_{\odot}$ or it may put strong upper limits on the number of such objects. ${ }^{5}$

Microlensing projects have been instrumental in determining the quantity of dark halo objects that contribute to dark matter in the galactic halo. Studies by the MACHO and EROS groups (Alcock et al. 1998, Alcock et al. 2000 and Lasserre et al. 2000) found a very high number of microlensing events occurring towards the Galactic Bulge, which could not possibly be sourced by the visible mass of the galactic halo. These microlensing events have been attributed to non-visible dark halo objects, however, the quantity of dark halo objects required to produce these extra microlensing events corresponds to only approximately $20 \%$ of the mass in typical halo models. The lower than expected number of microlensing events have ruled out the main candidates $\left(10^{-6} M_{\odot}-10^{2} M_{\odot}\right.$ mass objects) for Baryonic dark matter in the Milky Way.

\subsubsection{Planetary search}

The lightcurves of microlensing events can be altered by massive objects located at approximately the Einstein ring radius from the lensing star. Therefore, any binary companion of the lensing star at the Einstein radius will cause the resulting microlensing lightcurve to be significantly perturbed from the point lens microlensing model. In 1991, Mao and Paczyński estimated that approximately $10 \%$ of microlensing events toward the Galactic Bulge would show a variation from the point lens model (discussed in the next section). Variations such as these are now known to be caused by binary companions, which range in size from stellar objects down to planetary companions. This is currently the only method available that is sensitive enough to detect Earth-sized extrasolar planets from the ground. Recent studies of the microlensing event MACHO 98-BLG-35 by the MOA and MPS projects utilised this method and were able to successfully model the microlensing event's lightcurve with one or more planetary companions (Rhie et al. 2000 and Bond et al. 2002b). Theoretical studies into high-magnification ${ }^{6}$ microlensing events (Griest \& Safizadeh 1998) have suggested that Jupiter mass planets with an orbital radius between 0.6 and 1.6 of the Einstein radius will have detection probabilities of approximately $100 \%$.

\footnotetext{
${ }^{5}$ Paczyński 1986a.

${ }^{6}$ Microlensing events with amplitudes greater than 20 are deemed to be high magnitude.
} 


\subsection{Physics of microlensing}

Einstein's theory of General Relativity predicts that light passing close to a massive object will be deflected by an angle $\nu$. The equation formulated by Einstein to calculate $\nu$ was used to derive the time dependent amplitude equation for light from a lensed object, and detailed derivations of the point source microlensing equation can be found in Refsdal (1964), Paczyński (1986) and Griest (1991). The point source microlensing equation assumes that both the source and lensing objects are point-like, and that the transverse velocity $\left(v_{t}\right)^{7}$ is constant. The amplitude $(A)$ of light from a lensed object is given by

$$
A=\frac{u^{2}+2}{u\left(u^{2}+4\right)^{1 / 2}}, \quad u=\frac{r_{0}}{R_{E}}
$$

Where $r_{0}$ is the radial distance in the lensing plane between the lensing object and the source object and $R_{E}$ is the Einstein ring radius. The radial distance $r_{0}$ changes with time as the two objects move relative to each other from the Earth's reference frame and is defined by the equation

$$
r_{0}^{2}(t)=\beta^{2} R_{E}^{2}+\left[v_{t}\left(t-t_{0}\right)\right]^{2}
$$

Equation 4.1 shows $u$ as it is calculated at some instant of time, however, the distance $r_{0}$ is a function of time, thus $u$ can also be rewritten as a function of time $u(t)$

$$
u(t)=\frac{r_{0}}{R_{E}}=\sqrt{\beta^{2}+\left(\frac{t-t_{0}}{t_{E}}\right)^{2}}
$$

where $t_{E}$ is the time for the relative motion of the lens and the source to move one Einstein ring radius

$$
t_{E}=\frac{R_{E}}{v_{t}}
$$

The Einstein ring radius $\left(R_{E}\right)$ defines the size of the circular image formed when the source object, the lensing object and the observer are perfectly aligned. This value is dependent on the distance between the observer and the source object $\left(D_{S}\right)$, the distance between the lensing object and the source object $\left(D_{L}\right)$ and the mass of the source object

\footnotetext{
${ }^{7}$ The transverse velocity is the motion of the lensing object perpendicular to the line between the observer and the source star.
} 
$(M)$, where the distances are measured in kiloparsecs and the mass is measured in solar masses. The equation for the Einstein ring radius is

$$
R_{E}=\sqrt{\frac{4 G M}{c^{2}} \frac{D_{L}\left(D_{S}-D_{L}\right)}{D_{S}}}
$$

The impact parameter $\beta$ is the minimum distance between the lensing object and the observer-source object line, and is represented as a fraction of the Einstein ring radius. The variable $t_{0}$ is the time when the lensing object is at the minimum distance to the observer-source object line. The minimum value of $u(t)$ is found when time $t$ is equal to $t_{0}$, the time when the source object passes closest to the lensing object on the plane of the lensing object. The variable $t_{E}$ is the time taken from $t_{0}$ to when the source object passes the Einstein ring radius.

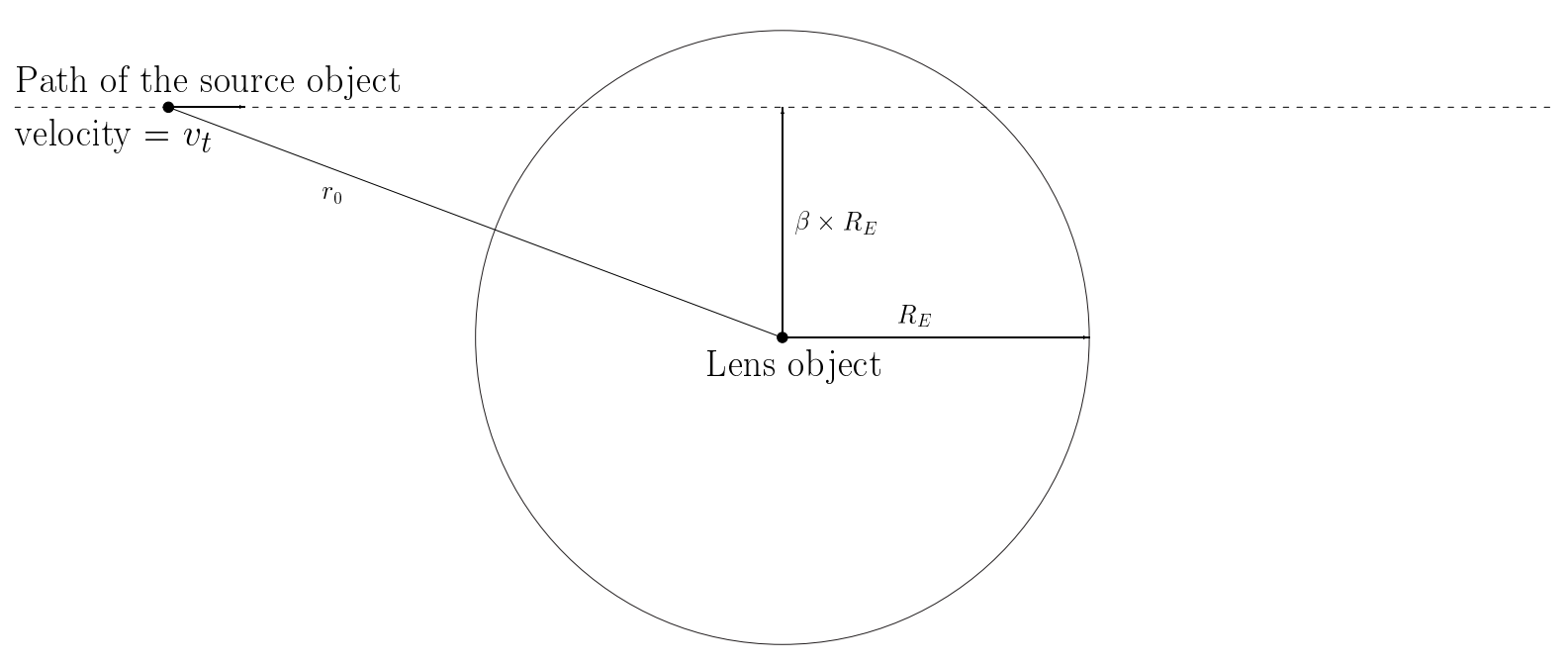

Figure 4.1: Shows the separation of the lens and source object in the plane of the lensing object.

A change in the relative velocity of the source object, lensing object or the observer contributes equally to the transverse velocity $v_{t}$. Although it is impossible to distinguish which of the three points has changed its velocity, the movement of the Earth around the Sun does, in some situations, change $v_{t}$. The parallax microlensing equation was developed in order to model lightcurves where the change in $v_{t}$ was believed to be caused by the motion of the Earth. In most cases, the distances between the observer and the source and lensing objects of microlensing events are large. These large distances cause high transverse velocities $v_{t}$, relative to the change in velocity of the Earth in its orbit around the Sun. When the source and lensing objects are closer to the observer, however, 
Point source microlensing model

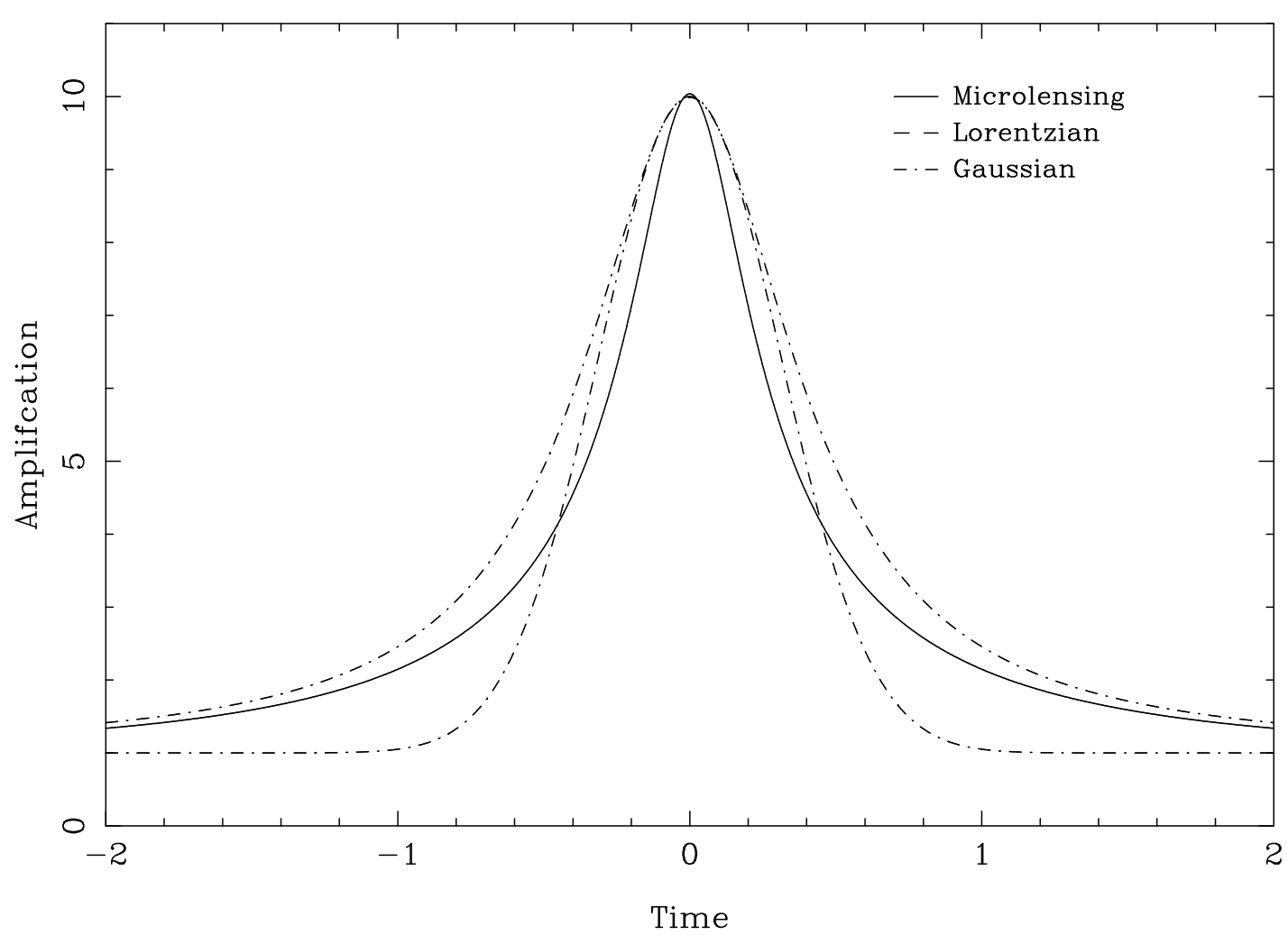

Figure 4.2: The figure shows the microlensing curve in comparison to the Lorentzian and Gaussian bell shaped curves.

the motion of the Earth becomes a significant fraction of $v_{t}$. The parallax microlensing equation (Alcock et al. 1995) models the lightcurve produced when the motion of the earth affects $v_{t}$ by replacing the equation for $u(t)$ with

$$
\begin{aligned}
u^{2}(t)= & u_{0}^{2}+\omega^{2}\left(t-t_{0}\right)+\alpha^{2} \sin ^{2}\left(\Omega\left(t-t_{c}\right)\right)+ \\
& 2 \alpha\left(\Omega\left(t-t_{c}\right)\right)\left(\omega\left(t-t_{0}\right)\right) \sin \theta+u_{0} \cos \theta+\alpha^{2} \sin ^{2} \beta \cos ^{2}\left(\Omega\left(t-t_{c}\right)\right)+ \\
& 2 \alpha \sin \beta \cos \left(\omega\left(t-t_{c}\right)\right)\left(\omega\left(t-t_{0}\right) \cos \theta-u_{0} \sin \theta\right)
\end{aligned}
$$

The variable $u_{0}$ is similar to the variable $\beta$ in the point source constant velocity model, however, the minimum distance occurs between the lensing object and the Sun-source object line. The $u_{0}$ variable is represented as a fraction of the Einstein ring radius. The variable $\omega$ is calculated from $\omega=2 / t_{0}$, where $t_{0}$ has the same definition as in the point source constant velocity model. $t_{c}$ is the time when the Earth is closest to the Sun-source object line. The value $\theta$ is the angle between the vector $v_{t}$ and the North ecliptic axis. 
The parameters $\alpha$ and $\Omega$ are calculated by the equations

$$
\alpha=\frac{\omega(1 A U)}{\tilde{\mathrm{V}}}\left(1-\epsilon \cos \left(\Omega_{0}\left(t-t_{p}\right)\right)\right)
$$

and

$$
\Omega\left(t-t_{c}\right)=\Omega_{0}\left(t-t_{c}\right)+2 \eta \sin \left(\Omega_{0}\left(t-t_{p}\right)\right)
$$

where $\Omega_{0}=2 \pi \mathrm{yr}^{-1}, t_{p}$ is the time of perihelion ${ }^{8}, \tilde{\mathrm{v}}=v_{t} /(1-x)$ is the lensing object's velocity projected to the Solar position and $\epsilon=0.017$ is the Earth's orbital eccentricity.

The first microlensing event successfully modelled with the parallax microlensing equation was detected by the MACHO project in 1994 (Alcock et al. 1995), which allowed the MACHO group to discover more about the event than would have been possible with the point source microlensing equation. By assuming various parameters, the MACHO group were able calculate the probable values for the mass of, and the distance to, the lensing object of $D_{\text {lens }}=1.7_{-0.7}^{+1.1} \mathrm{kpc}$ and $M_{\text {lens }}=1.3_{-0.6}^{+1.3} \mathrm{M}_{\odot}$.

The point source microlensing equation assumes that both the source and lensing objects are point-like, and that $v_{t}$ is constant. The parallax microlensing equation is applied when $v_{t}$ is not constant. In 1994, astrophysicists Hans Witt and Shude Mao derived an equation for a finite size source object lensed by a point-like lensing object (Witt \& Mao 1994). The finite source equation was successfully used by the MACHO group, in collaboration with others (including the MOA group), to model the microlensing event MACHO 95-BUL-30 (Alcock et al. 1997). The most probable values for the mass of, and distance to the lensing object were determined to be $D_{\text {lens }}=6.57_{-2.25}^{+0.99} \mathrm{kpc}$ and $M_{\text {lens }}=0.53_{-0.35}^{+0.53} \mathrm{M}_{\odot}$.

\subsection{Modelling for microlensing curves}

A $\mathrm{C}++$ program was designed to determine the best model to fit the data of possible and simulated microlensing events. The program ran a grid search of the three variables $\beta, t_{0}$ and $t_{E}$ in the point source microlensing equation (Equation 4.3) and the baseline magnitude. This grid search calculated a value for the quality of the model (a $\chi^{2}$ value) in which a single parameter was varied, while the other parameters were kept constant. The quality of the model was calculated for every combination of the parameters within the range of values investigated, thus allowing the discovery and detailed investigation of

\footnotetext{
${ }^{8}$ The perihelion is the moment in Earth's orbit when it is closest to the Sun.
} 
the best fitting model. This method's primary disadvantage pertained to computational time, as a large number of iterations were needed to calculate the quality of the model for each combination of the parameters. The number of iterations made by program was the multiple of the number of values searched for each variable (typically 10), and the number of observations. ${ }^{9}$

The microlensing modelling program's first pass searched a large range of values for each variable and calculated a $\chi^{2}$ value to determine the quality of fit for each model investigated. After the completion of the first pass, the variable values associated with the lowest $\chi^{2}$ model were used as the starting values for the second pass. The range of variables which the second pass searched was reduced to $10 \%$ of the first pass, which resulted in the increased accuracy of the variable values associated with the lowest $\chi^{2}$ model. The number of passes performed by the modelling program was provided by the user, thus impacting upon the accuracy of model. Figure 4.3 and table 4.1 show the effects on the accuracy of the model; the number of passes is increased with the microlensing modelling program.

\begin{tabular}{|l|r|r|r|r|r|r|}
\hline & & \multicolumn{5}{|c|}{ Number of passes } \\
\hline \multicolumn{1}{|c|}{ Variable } & Simulated values & One & Two & Three & Four & Five \\
\hline \hline Unlensed flux & 15000 & 14000 & 15060 & 15015 & 15044 & 15044 \\
\hline$\beta$ & 0.85 & 0.8 & 0.96 & 0.896 & 0.874 & 0.868 \\
\hline$t_{0}$ & 1305 & 1310 & 1301 & 1304.1 & 1304.11 & 1304.27 \\
\hline$t_{e}$ & 22.5 & 40 & 25 & 23.3 & 22.01 & 21.95 \\
\hline Amplification & 1.47388 & 1.4 & 1.36 & 1.427 & 1.448 & 1.454 \\
\hline$\chi^{2} /$ DOF & - & 6.194 & 1.631 & 1.529 & 1.511 & 1.508 \\
\hline
\end{tabular}

Table 4.1: The table shows the effect of increasing the number of passes of the microlensing modelling program with respect to the accuracy calculated. The bottom row contains values of $\chi^{2}$ per Degrees Of Freedom.

Lightcurves of microlensing events are difficult to simulate because the source star of an event does not fit into a particular class of star, and properties such as brightness and variability can take on any value. The simulations designed to test the microlensing modelling program used brightness values calculated from the point source microlensing equation, plus a random variance and error. The random variance of the brightness added

\footnotetext{
${ }^{9}$ The model curve, defined by the variable values must be compared to each observation to find a $\chi^{2}$ value.
} 

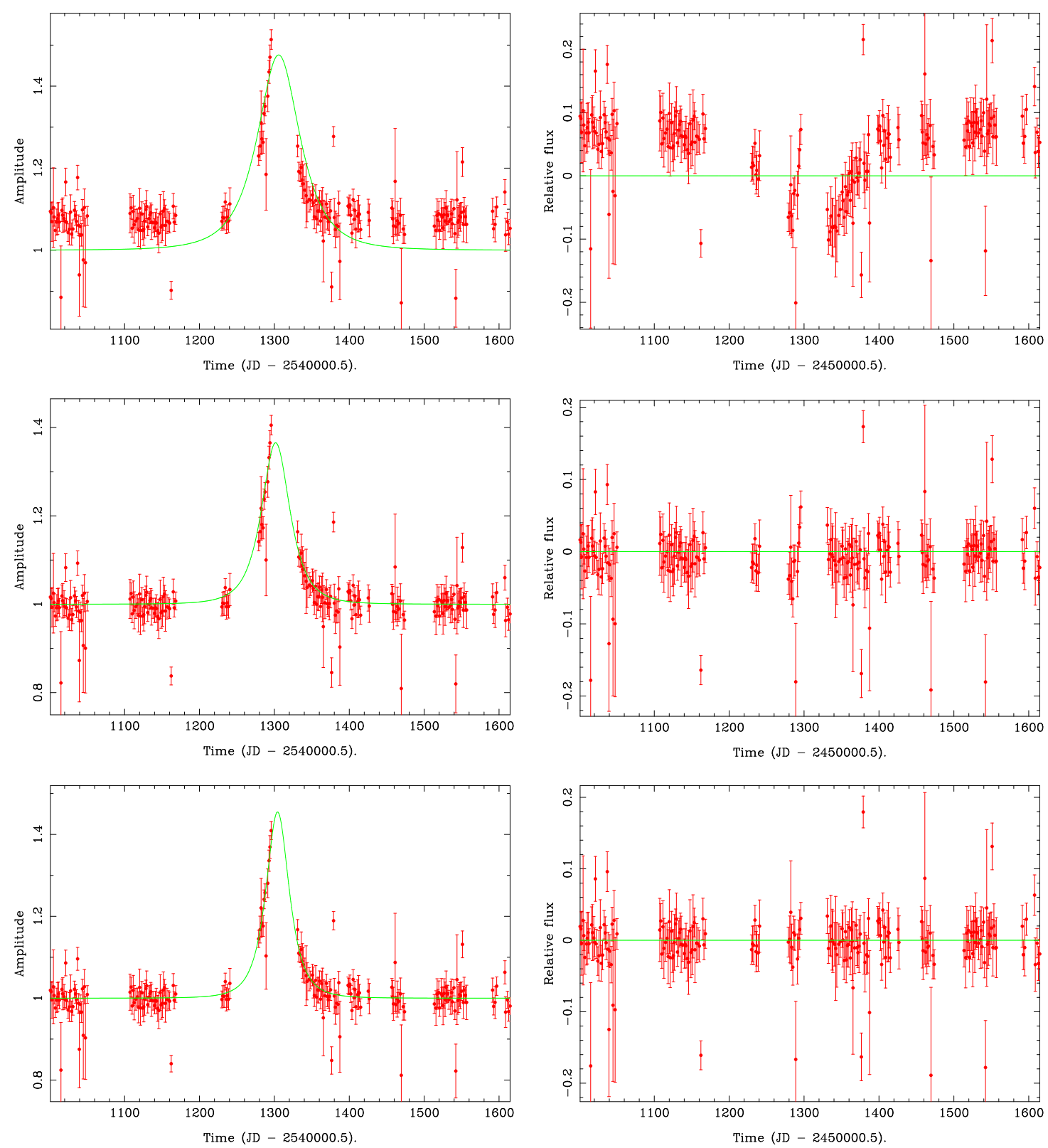

Figure 4.3: The figures show the results of the microlensing modelling program on simulated data for three different levels of accuracy. The plots on the left show the calculated model laid overtop the simulated data for one, two and five passes from top to bottom. The plots on the right show the difference between the fitted models and observed data for the associated plots on the left. 
to the point source equation was given an upper limit of $5 \%$ for $95 \%$ of the points and $20 \%$ for $5 \%$ of the points. The two upper limits on the variance allowed the simulation to include the average noise level in $95 \%$ of points and the massive variation that can occur due to errors in observation, reduction and analysis.

Point source microlensing model.

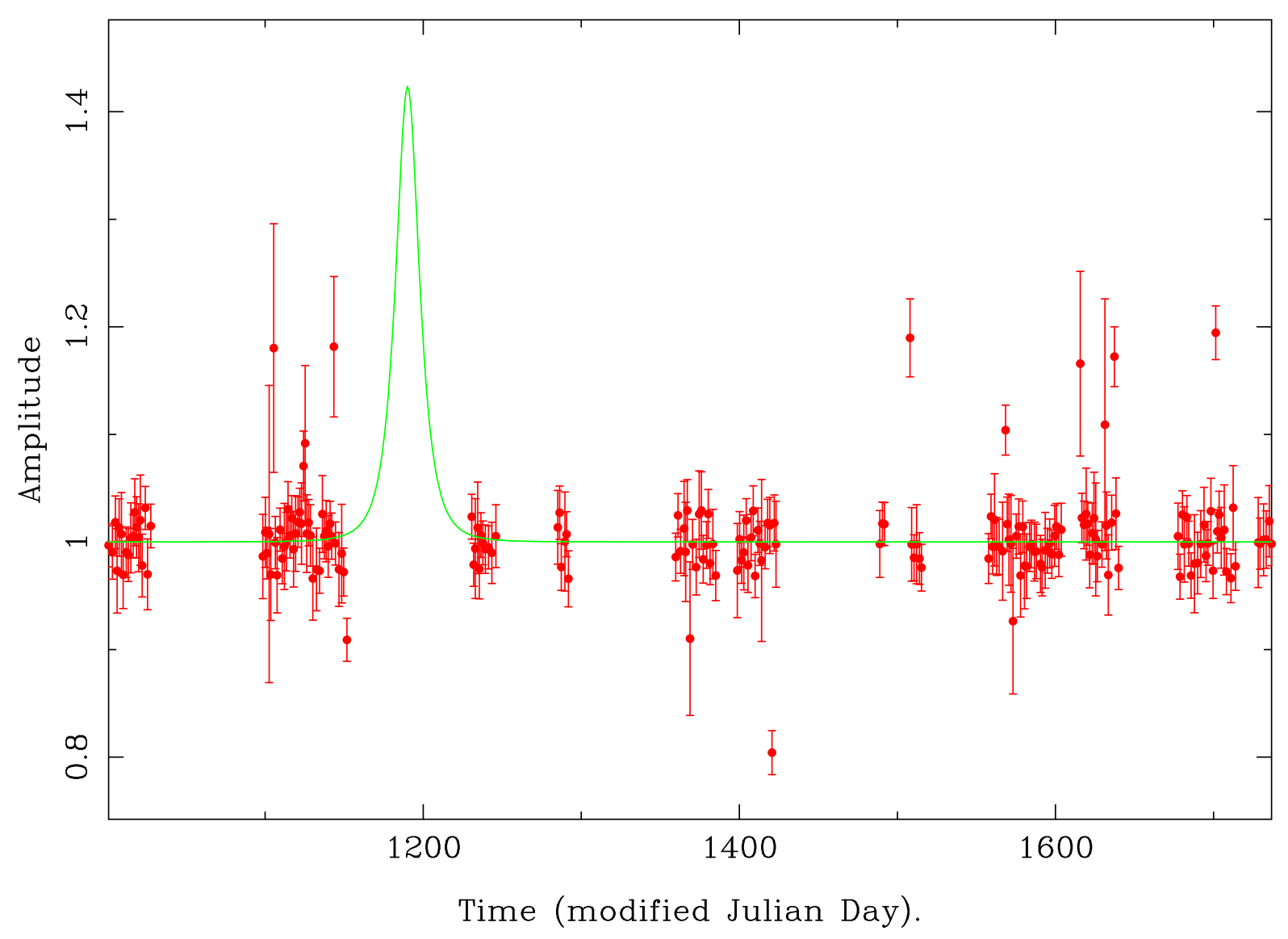

Figure 4.4: This lightcurve was simulated without a microlensing event, however, because of the large gaps in the data and the nature of the $\chi^{2}$ test, the lightcurve was modelled with a $\chi^{2} / \mathrm{DOF}$ of 1.89 , which suggested a good fit.

The data collected by the MOA project contains variations in brightness and time. Time differences between observations ranged from 1 hour to 3 months; one-hour separations were caused by the standard time taken to observe all the MOA fields and start a second set of observation on the same fields. Longer delays between observation were attributed to daylight hours, the full moon, cloudy weather and equipment failure. The time variations between successive observations were simulated by separating $95 \%$ of observations by a random distribution between 1 and 3 days and the remaining $5 \%$ of observations by a random distribution between 10 and 60 days. This separated most simulated observations by a small amount and also included larger separations to simulate 
the delays caused by equipment failure.

A problem was discovered with the $\chi^{2}$ test, when microlensing curves were fitted to lightcurves which were relativity constant and which had a large gap between two observations. The model minimized the $\chi^{2}$ value when it placed the microlensing event in the gap between observations, thus fitting the rest of the constant curve with the constant base line flux level of the microlensing model. Figure 4.4 shows how the $\chi^{2}$ test can produce incorrect models with good $\chi^{2}$ values. To remedy this problem, two requirements were added to the program: a minimum of 10 points located within one crossing time $\left(t_{e}\right)$ of the peak time $\left(t_{0}\right)$, and one point located within 2 days of the peak time.

\subsection{Microlensing events}

The microlensing modelling program was used to search the 1997 MOA LMC fields for potential microlensing events. For this search, the microlensing program was optimised to rapidly search through large numbers of stars. The accuracy of the initial pass of the microlensing modelling program was reduced, and upper limits were placed on the $\chi^{2}$ value after each pass. This resulted in an increase of speed of the program, and a decrease in the number of passes required to reject lightcurves which did not contain microlensing events. Because of the large variation in brightness which occurs during a microlensing event, the Welch and Stetson variability index was used to eliminate stars with low levels of variability.

While searching through the 1997 LMC data, the microlensing modelling program found 29 lightcurves which passed the criteria for microlensing events. After investigating the lightcurves by eye, six potential microlensing events were flagged for further analysis. The lightcurves of potential microlensing events microcurve1309, microcurve2173 and microcurve10751 (Figures 4.5, 4.6 and 4.9) showed an increased amount of scatter in brightness close to the peak time $\left(T_{0}\right)$ of the best fitted microlensing models. Due to the scatter in the brightness, the nature of the events are unclear. The most probable source for the variation in brightness is an intrinsic variability of the star, however, analysis of longer time series lightcurves for further periodic variations would be necessary in order to confirm this.

Figure 4.7 shows a potential microlensing event lightcurve (microcurve4333) with an increase in brightness occurring at approximately 640 days which has been modelled as microlensing event with the point source microlensing equation. However, the lightcurve 
also shows a slight increase in brightness through both MOA-red and MOA-blue observations toward the end of the time series (approximately 750 days). This increase in brightness suggests that the main increase in brightness, modelled as a microlensing event, was not a singular event, but part of a larger sequence of brightness variations not caused by microlensing.

The use of multiple filters in the search for microlensing events, is crucial in order to distinguish microlensing events from long period variables. Microlensing has an achromatic effect on the lightcurves of stars, and increases the brightness at different wavelengths by the same amount. Figures 4.10 and 4.8 show the potential microlensing events microcurve6344 and microcurve12674. The MOA-red filter observations of microcurve12674 cannot be modelled adequately by the point source microlensing equation, however, they can be suitably modelled by the parallax microlensing equation. Analysis of observations collected through the MOA-red filter alone may have resulted in the classification of these events as real microlensing events. However, the multiple filter observations of these lightcurves show that the events are not achromatic and, therefore, cannot be microlensing events. The sole use of a single waveband may accept microlensing candidates which would be rejected if more than one waveband was observed. This is significant for the current MOA single waveband detection strategy.

The microlensing modelling program was also used to model microlensing events previously detected by the MOA project. This modelling was conducted to test the program on the microlensing lightcurves, which had been previously analysed. During the testing, an adaption of the microlensing program, which was based on the parallax microlensing equation (equation 4.6), was created to model the lightcurve of ngb1-2-2717. This adapted program showed that the data could be modelled with a better quality of fit by the parallax microlensing equation than the point-source equation. 


\subsubsection{The 1997 LMC data}
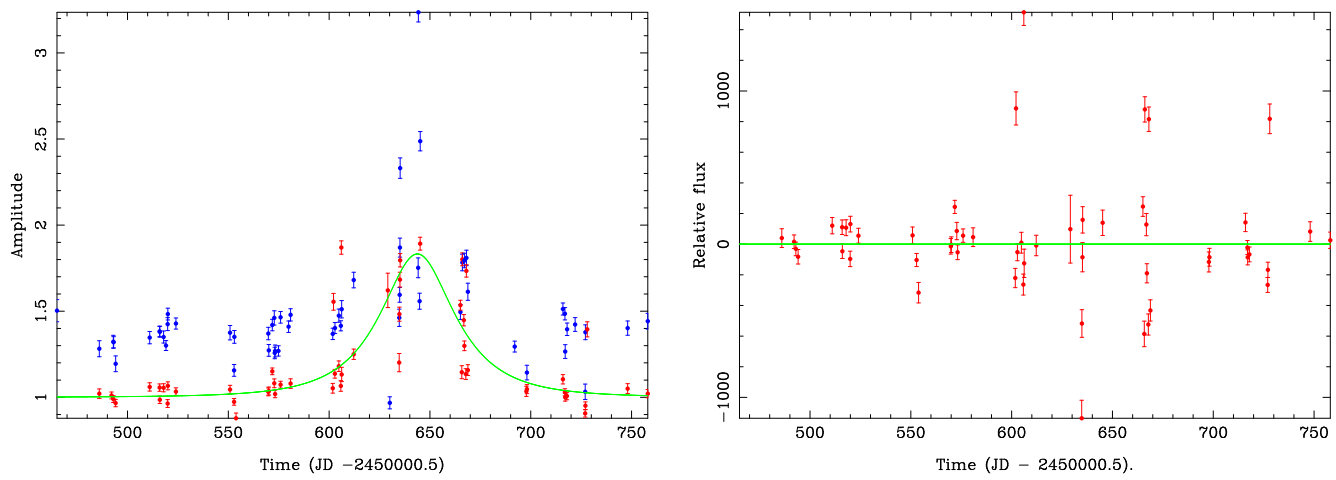

Figure 4.5: The MOA-red and MOA-blue lightcurves of the potential microlensing event microcurve1309 with the best fit point source microlensing model (green curve) for the MOA-red observations is shown on the left, and the residual values of the MOA-red observations subtracted from the best fit point source model on the right.
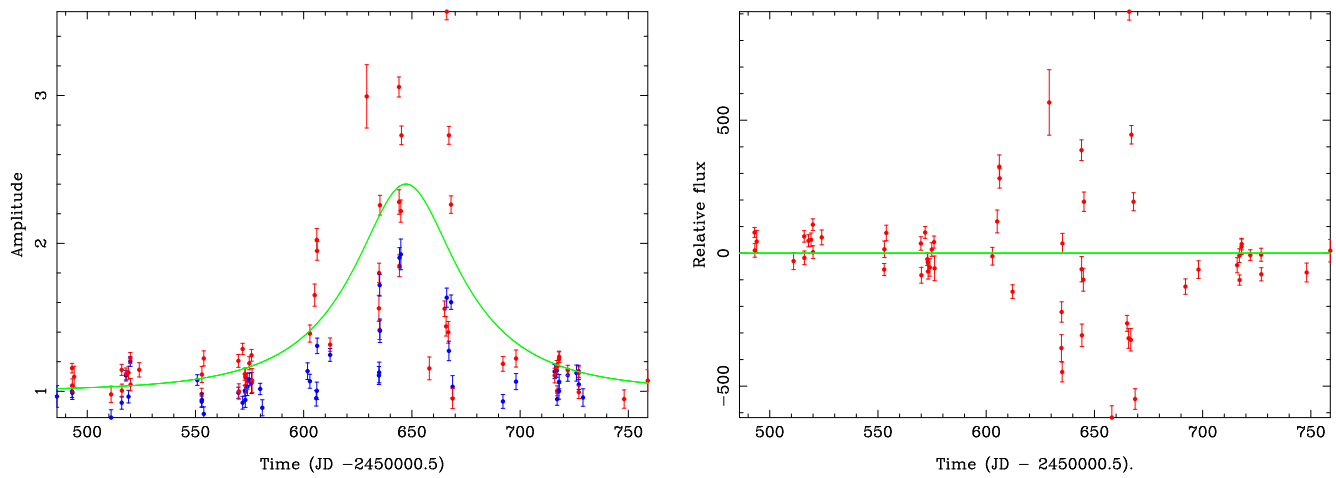

Figure 4.6: The MOA-red and MOA-blue lightcurves of the potential microlensing event microcurve2173 with the best fit point source microlensing model (green curve) for the MOA-red observations is shown on the left, and the residual values of the MOA-red observations subtracted from the best fit point source model on the right.
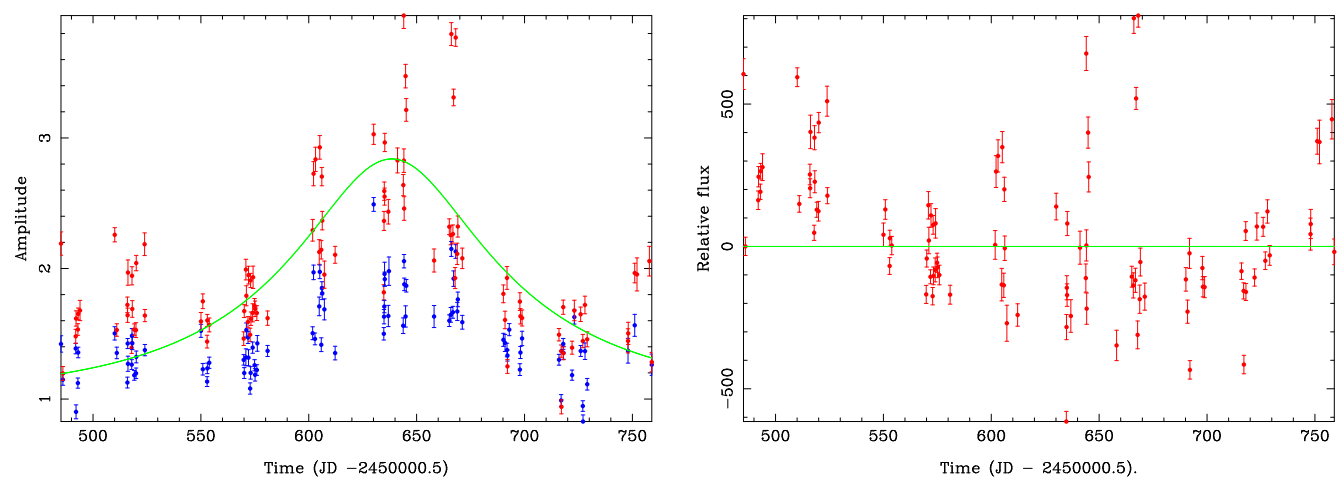

Figure 4.7: The MOA-red and MOA-blue lightcurves of the potential microlensing event microcurve4333 with the best fit point source microlensing model (green curve) for the MOA-red observations is shown on the left, and the residual values of the MOA-red observations subtracted from the best fit point source model on the right. 

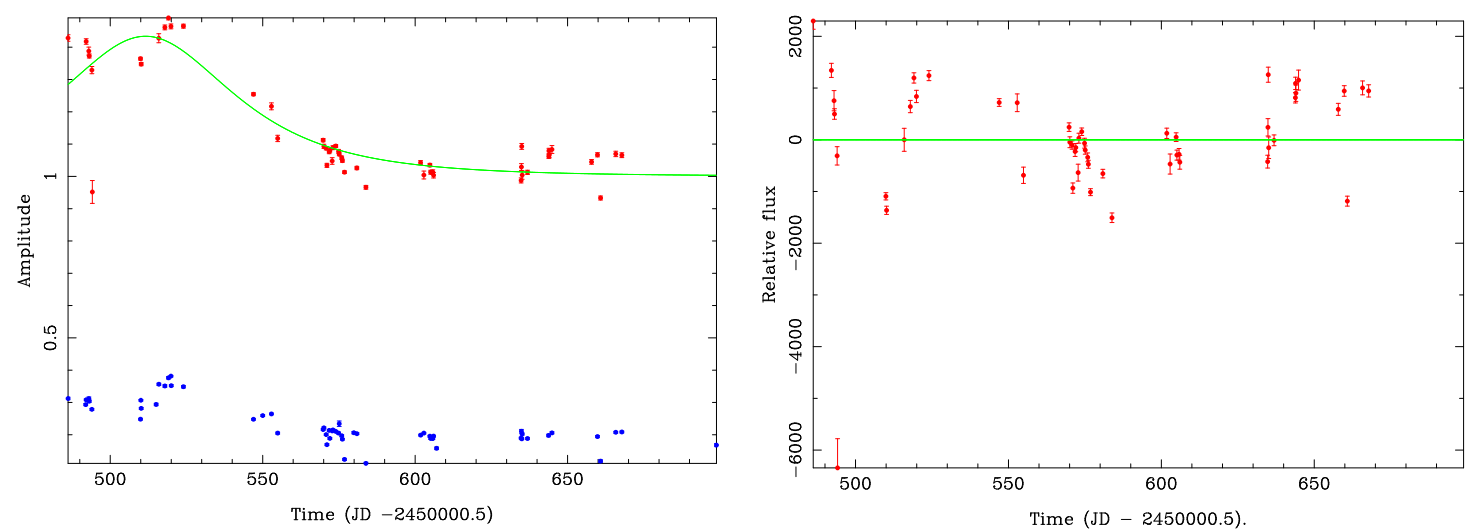

Figure 4.8: The MOA-red and MOA-blue lightcurves of the potential microlensing event microcurve6344 with the best fit point source microlensing model (green curve) for the MOA-red observations is shown on the left, and the residual values of the MOA-red observations subtracted from the best fit point source model on the right.
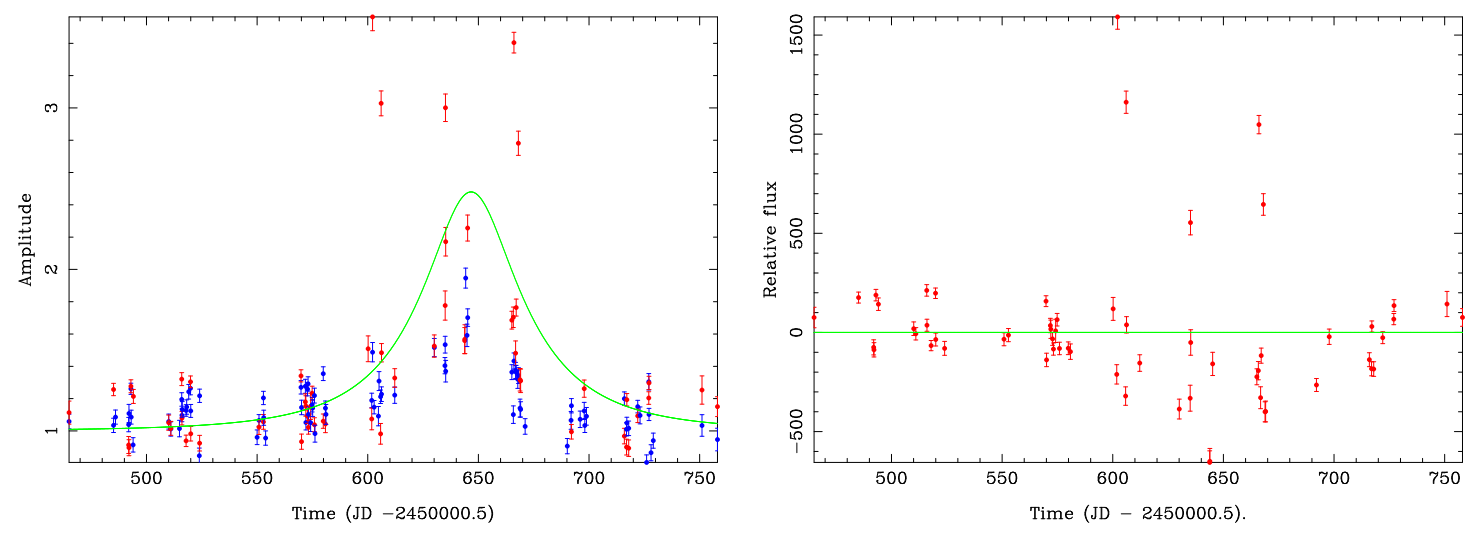

Figure 4.9: The MOA-red and MOA-blue lightcurves of the potential microlensing event microcurve10751 with the best fit point source microlensing model (green curve) for the MOA-red observations is shown on the left, and the residual values of the MOA-red observations subtracted from the best fit point source model on the right.
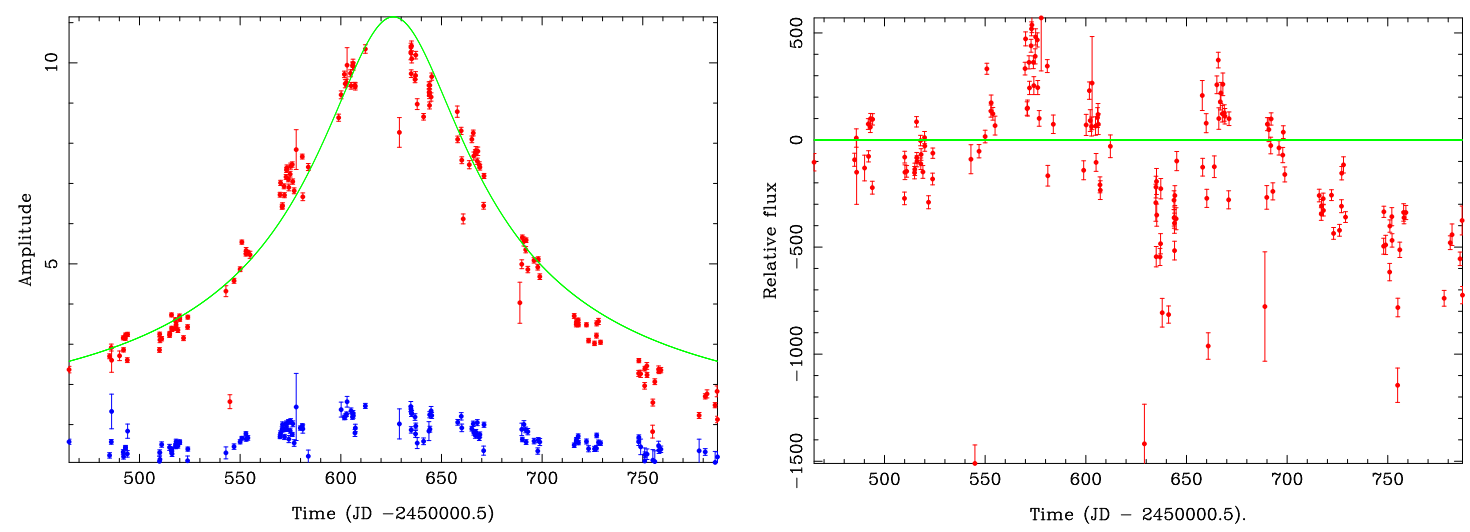

Figure 4.10: The MOA-red and MOA-blue lightcurves of the potential microlensing event microcurve12674 with the best fit point source microlensing model (green curve) for the MOA-red observations is shown on the left, and the residual values of the MOA-red observations subtracted from the best fit point source model on the right. 


\subsubsection{The 2000 Galactic Bulge data}
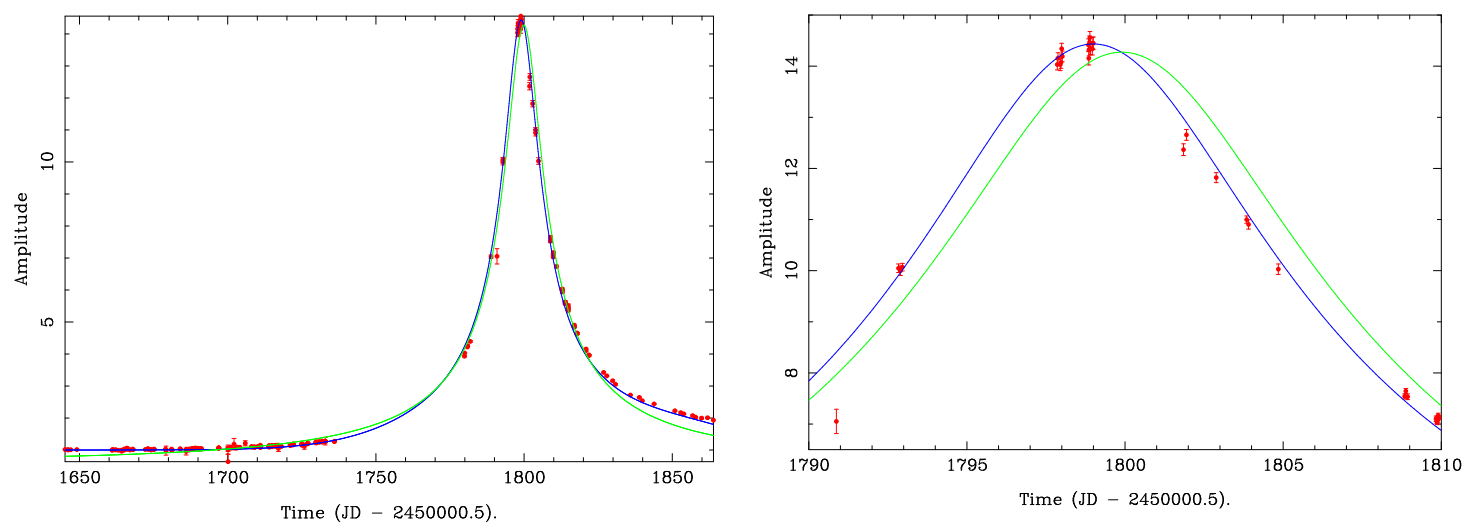

Figure 4.11: Lightcurve of the microlensing event ngb1-2-2717. The green curve represents the best fit point source microlensing model and the blue curve represents the best fit parallax microlensing model.
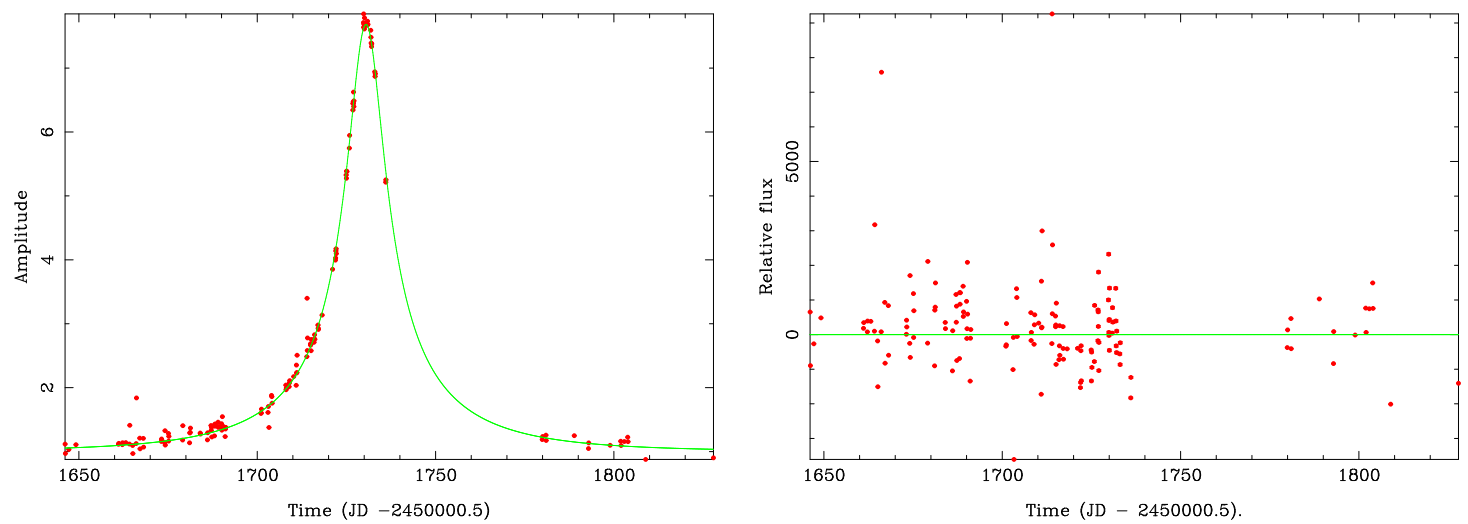

Figure 4.12: Lightcurve of the microlensing event ngb11-2-1142. The lightcurve with the best fit point source microlensing model for the MOA-red observations is shown on the left, and the residual values of the MOA-red observations subtracted from the best fit point source model on the right. 


\section{Chapter 5}

\section{Planetary transits}

\subsection{Extrasolar planets}

A planet can be broadly defined by the physical limits for nuclear burning in a selfgravitating object. The minimum mass limit for deuterium burning is approximately twelve times the mass of Jupiter $\left(12 M_{J u p}\right)$, and has been used to place an upper limit on the mass of a planet. Objects with a mass greater than $12 M_{\text {Jup }}$ are classified as brown dwarfs. These objects have an upper limit associated with lower mass limit for sustained hydrogen-burning, which is $8 \%$ of the Sun's mass $\left(0.08 M_{\odot}\right)$.

\subsubsection{Detection methods}

\section{Radial velocity method}

Gravitationally coupled objects orbit each other about a common centre of mass. Therefore, stars with planetary companions have a motion imparted about the centre of mass of the star planet system. The line of sight motion of the host star can be determined from the Doppler effect, where the light emitted from a star moving away from the Earth will be Doppler shifted towards the red end (longer wavelength) of the spectrum and light emitted from a star moving towards the Earth will be Doppler shifted towards the blue end (shorter wavelength) of the spectrum. The amount of Doppler shift detected from a star is directly related to the velocity of the star along the Earth-star line of sight. An example of the motion imparted on a star by an orbiting planet is the $12 \mathrm{~m} / \mathrm{s}$ velocity amplitude of the Sun induced by Jupiter. The presence of more than 60 planets around stars other than the Sun have been inferred using the radial velocity method to date. Although the radial velocity method is the most successful procedure for discovering extrasolar planets, the method can only determine $m \sin i$, where $m$ is the mass of the planet and $i$ is the inclination of the plane of the planetary orbit relative 
to the Earth ( thus $i=90^{\circ}$ corresponds to viewing the orbit edge on). Therefore, the radial velocity method can only determine a lower bound for the mass of a planetary companion.

Various projects are closely examining the extrasolar planets discovered by the radial velocity method for signs of planetary transits (section 5.1.2). If an extrasolar planet does transit its host star, the orbit inclination relative to the Earth is approximately $i=90^{\circ}$. The determined orbital inclination $i$, can be used in the equation $m \sin i$ to calculate the actual mass of the planet.

\section{Astrometry}

The principle behind the astrometric method of detecting extrasolar planets is the same as the radial velocity method. Gravitationally coupled objects impart motion about the object's centre of mass, however, the astrometry method directly observes the motion imparted by the planet in the star. By observing the position of a star relative to surrounding background stars, the motion of the star can be detected. Gravitationally coupled stars display periodic movement which is caused by the coupled object, yet the movement of a star due to a planetary sized object is difficult to detect from great distances. Thus, this technique can only be used on stars that are less than approximately 40 lightyears from the Earth.

\section{Direct Imaging}

Direct imaging of an extrasolar planet requires the detection of light reflected from the surface of the planet. This method of observation is similar to the method used to observe solar system planets. The quantity of light reflected by a planet is a small fraction of the total light emitted by the planet's host star, therefore, the light reflected by the planet would be swamped by the light from the star. For direct imaging of extrasolar planets to be a viable method of detection, the quantity of the light observed from the host star must be reduced without affecting the light reflected by the planet. Interference methods, combined with observing from space, will be required to achieve this.

\subsubsection{Planetary transits}

Planetary transit events are observed when a planetary object passes directly between an observer and the planet's host star. For observers on Earth, only Mercury and Venus can be observed transiting the Sun, as they are the only planets that pass between the Earth and the Sun. 
In 1621, the German mathematician Johannes Kepler correctly predicted that the planetary transits of both Mercury and Venus would occur in the year 1631 and that transits of Venus would occur once every 120 years. Three people observed the 1631 transit of Mercury, however, there were no observations of the transit of Venus, as it occurred after the Sun had set over Europe. In 1639, the British astronomer, Jeremiah Horrocks, predicted another transit of Venus would occur that year, eight years after to Kepler's predicted transit. The 1639 transit was observed by Horrocks, who also calculated that transits of Venus occurred in pairs, eight years apart, with 120 years separating successive pairs.

When a planetary transit is observed, there is a reduction in the intensity of light as the shadow of the planet (from the host star) passes over the observer. The intensity of light from the star is reduced by an amount proportional to the area occulted by the disk of the planet. The cross section of a planet is small relative to the cross section of a star, therefore, the reduction in intensity is a relatively small effect, approximately $2 \%$ of the total intensity from the star in the case of HD 209458 (Henry et al. 2000 and Charbonneau et al. 2000).

The simplest transit model is the geometric transit model, which is defined by a nonradiating non-transmitting planetary disk passing in front of a star of uniform brightness. However, this model ignores limb darking, which is the decrease in brightness of a star as the radius increases from the centre of the stellar disk. The limb darking model uses a stellar disk which consists of concentric rings that decrease in brightness from the centre of the disk. Data obtained from a planetary transit HD 209458 (figure 5.2) shows a lightcurve which can only be adequately fitted by the limb darking model (figure 5.1).

The STARE (STellar Astrophysics and Research on Exoplanets) project was founded in 1999. It is a group which aims to find evidence of extrasolar planets by searching for planetary transits in large fields of stars centred on the Galactic Bulge (Brown \& Charbonneau 1999). During August and September of 1999, members of the STARE project observed the planetary transit of the object HD209458 (Charbonneau et al. 2000). Observations were undertaken with a portable $10 \mathrm{~cm}$ Schmidt telescope, and images were produced using a Pixelvision $2034 \times 2034$ pixel CCD. The project initially undertook observations from Boulder, Colorado, USA, however, a new dome located on the island of Tenerife in the Canary Islands is currently under construction. Planetary transits were also searched for on stars with planetary companions previously discovered using the Radial Velocity Method. Eleven candidate stars were used by STARE in their search and were chosen because of the close proximity of the planetary companion to the host star 
(orbital separation $a<0.1 \mathrm{AU}$ ). The close-in massive extrasolar planets (hot Jupiters) were selected because of the higher probability that these planets would transit the host star.

Simulated Planetary Transit Curves

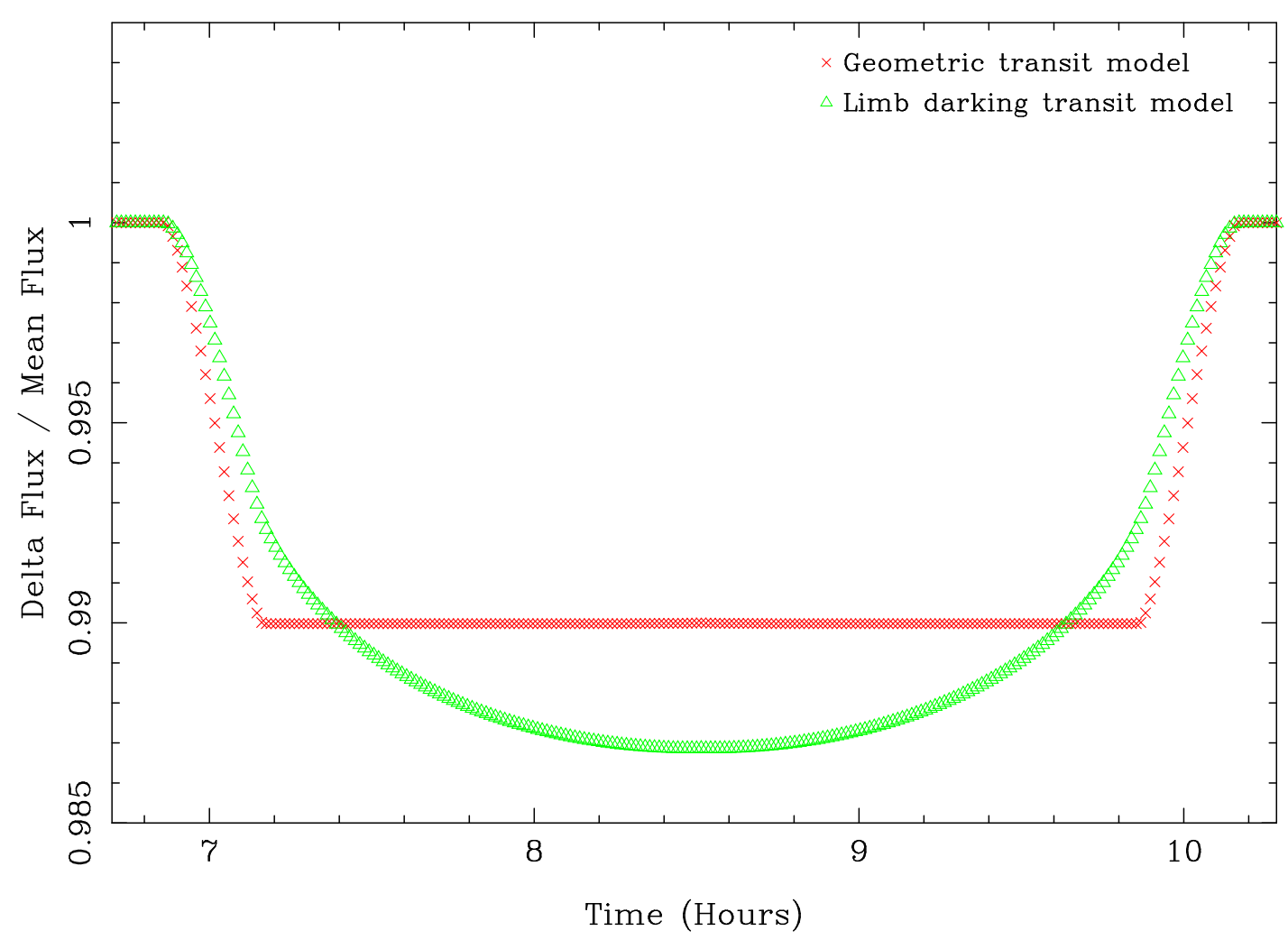

Figure 5.1: The figure shows the two planetary transit models with the same set of parameters for both models. The curves were generated by a transit modelling program which was developed by Denis Sullivan.

The probability that a planet will transit its host star depends upon the orbital plane of the planet relative to Earth. Planetary transits that are detectable from ground-based observations require the planet to have a large physical size relative to the host star, as this increases the reduction in brightness from the star when the planet transits in front of it. Moreover, the orbital radius of the planet must be small, as this increases the frequency of observable planetary transits. Hot Jupiter planets make good candidates in a search for planetary transits as they possess both close-in orbits and relatively large physical size.

Members of the STARE project observed the object HD 209458 for ten nights from August $29^{\text {th }}$ to September $16^{\text {th }} 1999$, and it was predicted that on two of the ten nights of observations, planetary transit events may be observed. Lightcurves that were collated 
from these two nights of observation displayed significant drops in brightness and were modelled as planetary transits using the parameters $R_{p}=1.27 R_{\text {Jup }}$ and $i=87.1^{\circ}$ (Charbonneau et al. 2000). Observations of HD 209458 made after the STARE project's initial observations (Castellano et al. 2000, Jha et al. 2000, Mazeh et al. 2000 and Brown et al. 2001) reinforced the existence of the planetary transit and increased the accuracy of the planet's parameters.

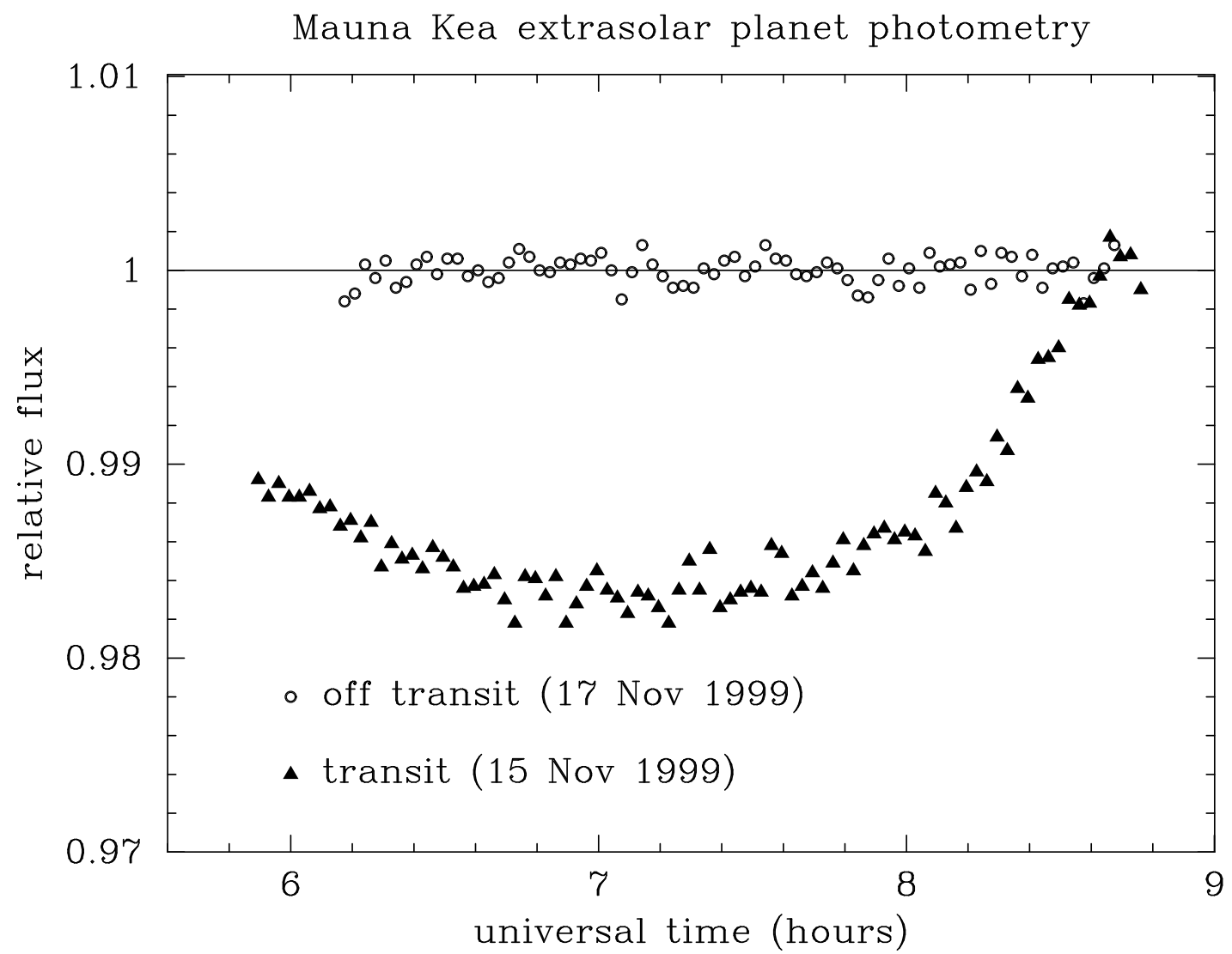

Figure 5.2: The figure shows the results of observations of the planetary transit of object HD 209458 recently obtained by Denis and Tiri Sullivan (Jha et al. 2000).

Another group that has searched for planetary transits is the large Hubble Space Telescope project, who conducted a search of the globular cluster 47 Tucanae in 1999 (Gilliland et al. 2000). Because of the rare nature of an extrasolar planetary transit event, any search of random stars must include tens of thousands of stars to increase the probability of success. The search for planetary transits in 47 Tucanae observed approximately 40000 stars in the densely populated globular cluster core. In the local neighbourhood of stars the frequency of hot Jupiter planets is approximately 1\%, and from this percentage approximately $10 \%$ will transit the host star. These figures suggest that for every 1000 stars surveyed, 1 will show signs of a planetary transit. The properties of the 47 Tucanae search combined with the statistics from the local neighbourhood of 
stars suggested that approximately 17 planetary transits should have been detected, yet none were found. This lack thereof has yet to be comprehensively explained. However, it is believed that the high metallicity ${ }^{1}$ of the surveyed stars or the crowded nature of the densely populated core of the globular cluster are key factors.

\subsection{Transit simulation and a phase space search for periodic transits}

Simulated lightcurves demonstrated how planetary transits would appear in the MOA data and suggested procedures which could be used to detect them. Currently, the only available method for predicting lightcurves of planetary transits in a large-scale ground based microlensing survey is simulation, because a successful search has yet to be undertaken.

The first lightcurve simulation was designed as a random distribution from a mean value, with a square 'well drop' that represented the drop in intensity caused by the planetary transit. The mean level used in the simulation was based on the average flux level for stars with standard deviations of less than $2 \%$ in one of the MOA fields (ngb 11). Simulated brightness was calculated using the square well model plus a random distribution limited to $\pm 1 \%$ of the mean brightness. The square well had a depth of $2 \%$ of the total flux and a width of $5 \%$ of the simulated planet's orbital period.

A series of detection programs were designed using the information collected from simulations. The first of these programs searched for observations located in a band of photometry, which ranged from $1.5 \%$ to $5 \%$ below the mean brightness. These points were assumed to be observations which had been taken during a planetary transit. This search program then calculated the minimum time between any two of the transit points and assumed that this time was approximately equal to the period or a integer multiple of the period. The first transit point in the time series was used as a base point, and all searches for subsequent transit points originated from it. During the next phase of the program, an area immediately surrounding the base point ( $\pm 5 \%$ of the period) and areas of the lightcurve which were integer number of periods from the base point $( \pm 5 \%)$ were searched for further transit points. The search was considered to be successful if $90 \%$ of the transit points were located an integer number of periods $( \pm 5 \%)$ from the base point. If the search using the original base point was unsuccessful, a new base point was

\footnotetext{
${ }^{1}$ The metallicity of a star is the abundance of elements more massive than helium.
} 
Transit with duration: 4.236 hours, and period: 4.412 days.

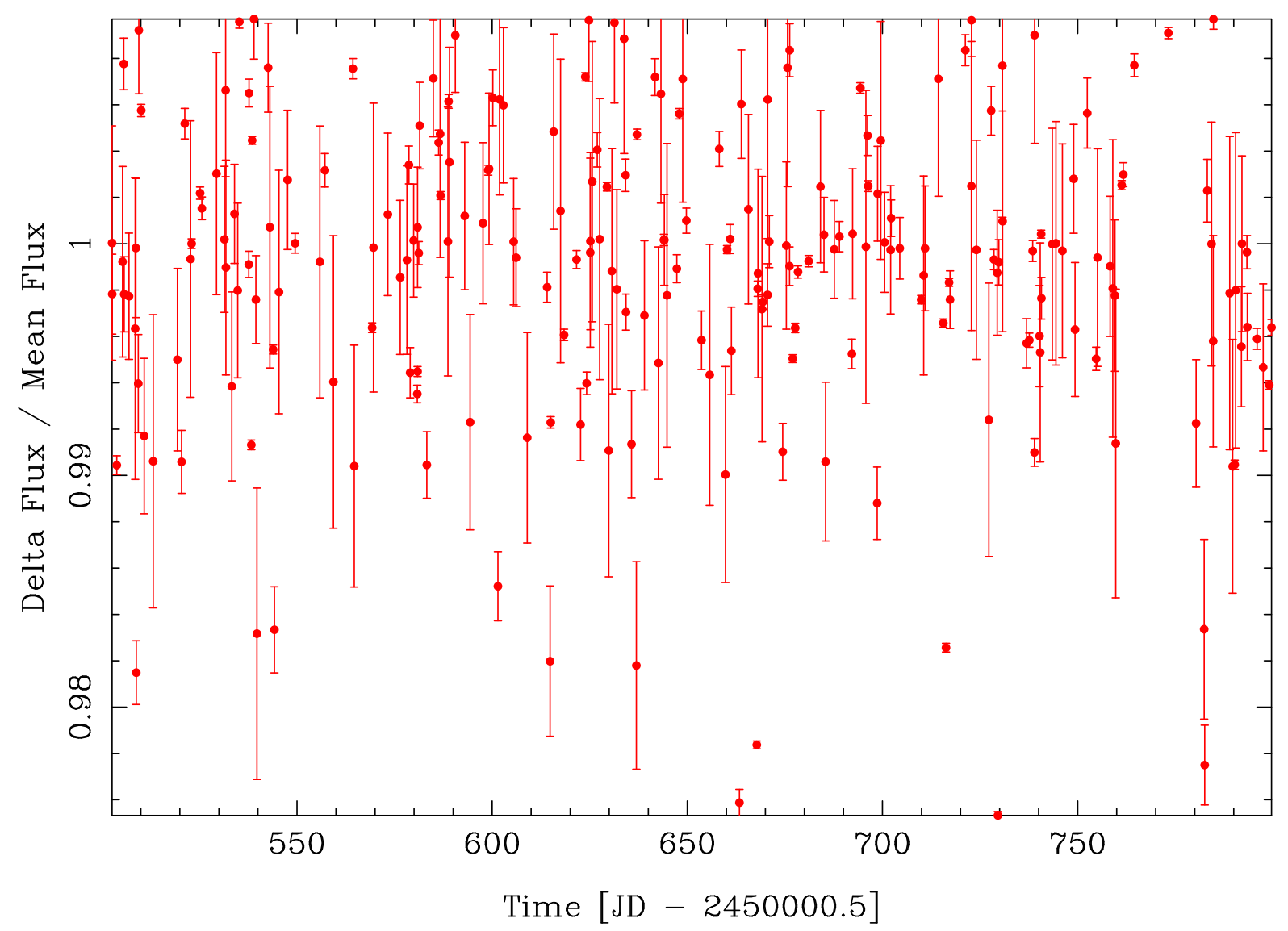

Figure 5.3: Simulated lightcurve of a planetary transit. The simulation had a 1\% random distribution in the brightness and a square well drop representing the transit. The transit drop had a $2 \%$ depth and a $5 \%$ width.

selected from the transit points and the process was repeated. The search program was successful when applied to the simulated lightcurves, but it ceased to work if non-transit points were found in the $-1.5 \%$ to $-5 \%$ band. Simulations which closely represented the MOA data contained brightness which varied more than the original simulations. They increased the number of observations which were found in the $-1.5 \%$ to $-5 \%$ band and reduced the probability of the discovery of transit lightcurves. As a result, adjustments were made to increase the success rate of the program: the success percentage of $90 \%$ was reduced, however, false detections passed through the search program, thus rendering it ineffective.

The second transit search program searched through phase space (figure 5.4), where the observation point has time $t$ which is expressed as $t=n T+t^{\prime}$ where $T$ is the trial period, $n$ is an integer and $t^{\prime}$ is the time from $n$ number of the periods to the observation point. This search process 'folds' all the observations points of the lightcurve into the 
first trial period $T$ and searches for periodic signals. The folded lightcurve is displayed using a periodogram which shows the first trial period with all the observation points folded into it, where time is represented by values of phase (phase $=t^{\prime} / T$ ).

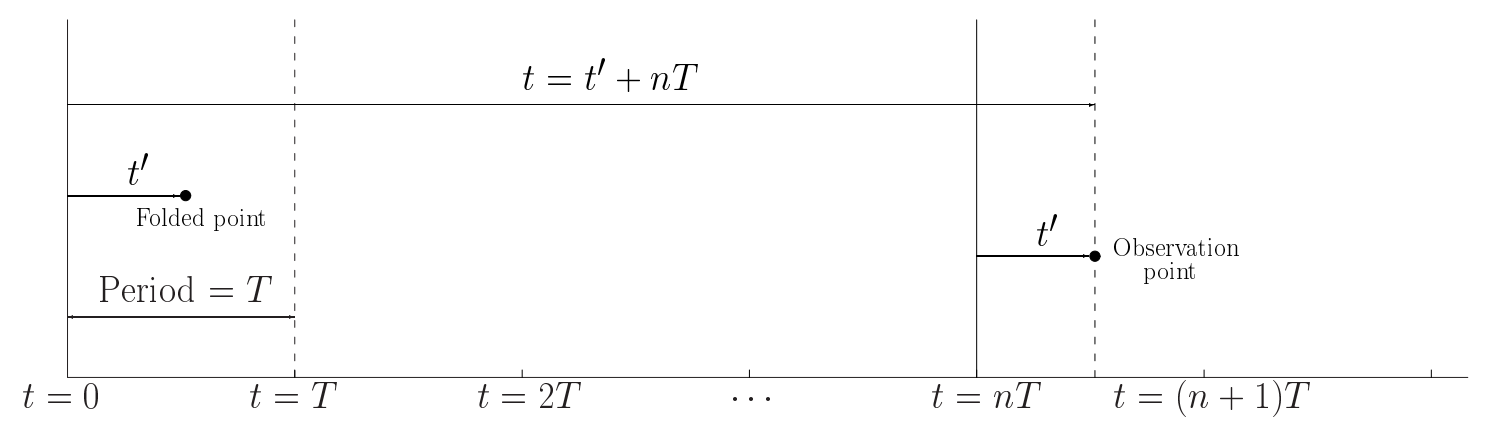

Figure 5.4: This figure shows how the observation point is folded back into the first period. Periodograms only show the first period with all lightcurves observations folded into it.

Lightcurve flux values were subtracted from the mean flux value and the time values were subtracted from the minimum time. The search through phase space was limited to periods between 1 and 8 days and lightcurve observations were folded into the first trial period (1 day). The program summed the products of the brightness values of the folded lightcurve observations and the corresponding flux values from the model curve for each observation. This summed value was referred to as the 'cross correlation' $(C)$ and is displayed in the equation

$$
C=\sum_{i=1}^{n} \operatorname{Model}(i) \times(\operatorname{Flux}(i)-\overline{\text { Flux }})
$$

A square well with a non-transit drop level of zero and a transit drop level of $2 \%$ of the mean flux value of the simulated star was used for the transit model. The model curve had a transit duration of $5 \%$ of the orbital period of the simulated planet.

$$
\operatorname{Model}(i)= \begin{cases}-0.02 \times \overline{\text { Flux }} & \text { if phase }=\text { transit portion } \\ 0 & \text { otherwise }\end{cases}
$$

The non-transit portion value of the transit model was zero, therefore, when it was multiplied by the folded lightcurve there was no contribution to the cross correlation value. The only contributions to the sum were the observations located over the transit portion of the model. If a transit event did not occur within the folded lightcurve, the flux values over the transit portion of the model were random values which varied about zero. When these values were multiplied by a constant value (the bottom of the well), the sum of the multiples tended toward zero, as the number of random points tend towards 
infinity. Because a lightcurve contains a finite number of points over the transit portion of the model, the summed values are normally distributed about zero (Figure 5.5).

Histogram of the Cross Correlation values

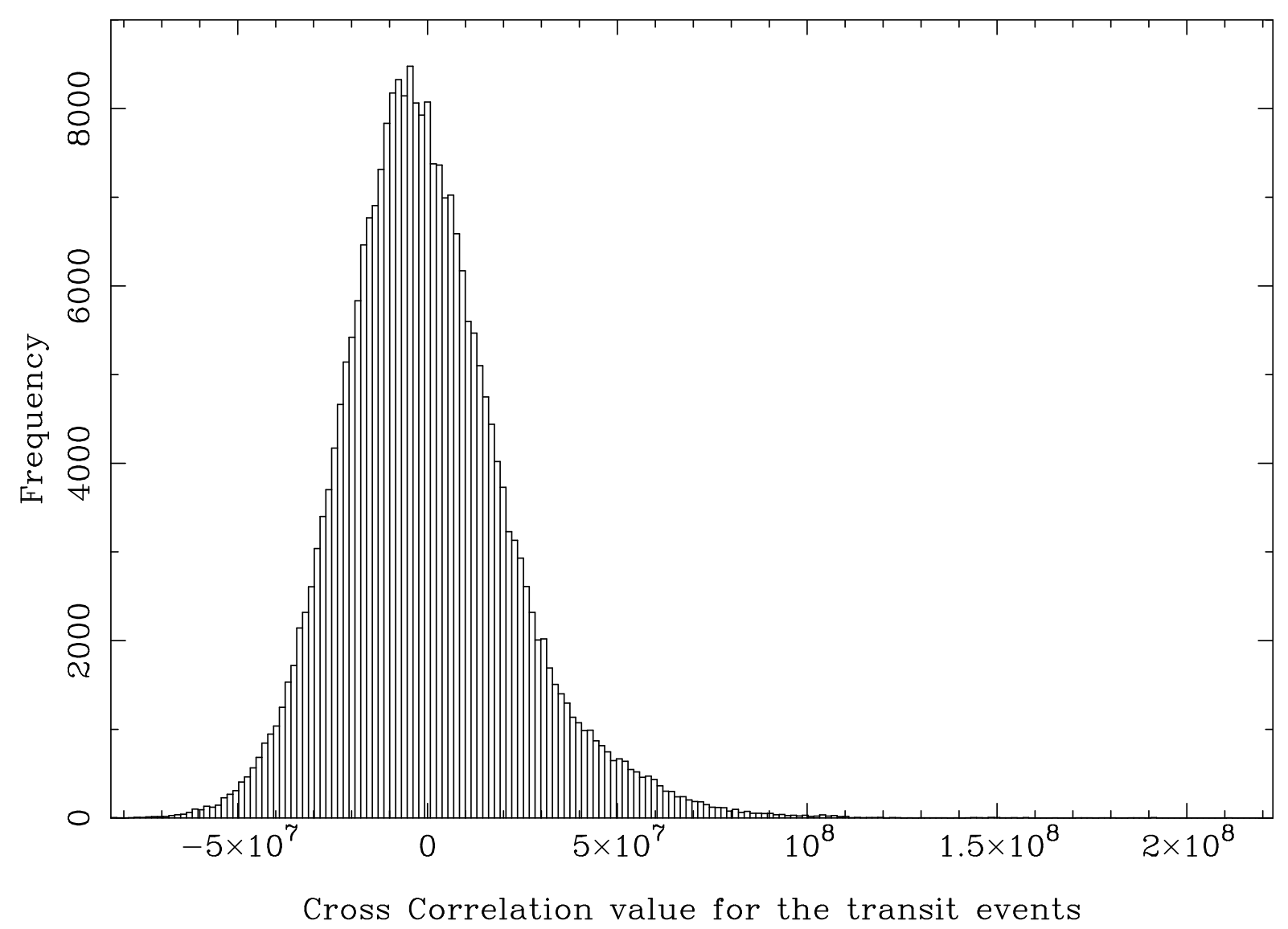

Figure 5.5: A histogram of the cross correlation values calculated for the transit simulation in figure 5.3. The cross correlation values are calculated for every iteration across phase space and are normally distributed about zero. The distribution extends toward the large positive values because of the interaction of the model 'well' and the lightcurve's transit, which results in large positive values.

When a transit event occurred within a folded lightcurve, all the transit points were grouped together as the folded period coincided with the orbital period of the planetary transit. The transit was detected when the transit portion of the model was moved over the grouped points. Both the folded lightcurve transit points and the model's square well have values below zero, thus the multiple of the two values produced a positive number. Large positive values of the product resulted when the values of the folded lightcurve's transit points were low. When the model curve and the transit event coincided in phase space it resulted in large and positive cross correlation values. The phase space search program looked for the period with the highest value of cross correlation for all iterations, and used this as the most probable transit period. To eliminate non-transit lightcurves, 
the search program set a lower limit for the maximum value of cross correlation, and lightcurves whose maximum cross correlation value did not reach this limit were rejected.

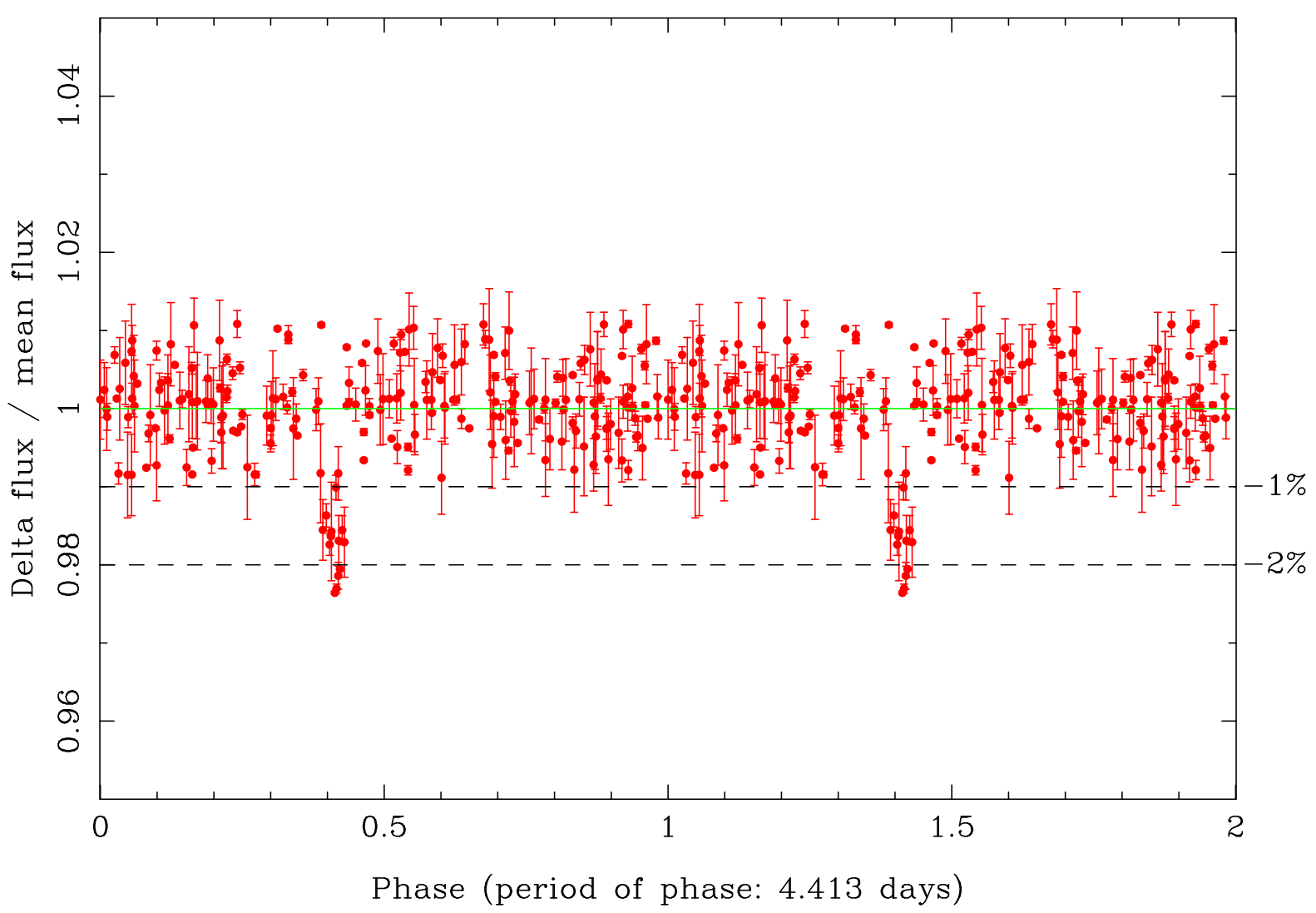

Figure 5.6: Shows the output of the period folding search program run on the simulated transit lightcurve in figure 5.3 .

Observations with brightness values that were below $97 \%$ of the mean brightness were considered to be too low to be real planetary transit events and were removed. The most probable period of candidate transit lightcurves was determined by the highest value of the cross correlation found for the trial periods. The phase space search program was initially designed to use a square well to model the transit event. This model was used in a coarse search to determine the most probable transit period, which in turn was used in a more detailed search around the value to determine a more precise period. Both the coarse search and detailed search used the square well model. Subsequent variations of the phase space search program used the same coarse search, however, a sixth order polynomial represented the transit event in the more accurate search, which is shown by the equation

$$
\operatorname{Model}(i)= \begin{cases}m\left(t-t_{c}\right)^{6}+F_{0} & \text { if phase }=\text { transit portion } \\ 0 & \text { otherwise }\end{cases}
$$


where $t$ is time, $t_{c}$ is the time at the center of the transit, $m$ is a constant that matches the transit portion of the model to the rest of the curve and $F_{o}$ is the flux level at the bottom of the well. The value for $m$ is calculated by making the model continuous at the transit/non-transit boundary at the edge of the transit well, and substituting the model transit depth $\left(0.02 \times F_{b}\right)$ and the transit duration $(0.05 \times T)$ into the equation

$$
F_{b}=m\left(t-t_{c}\right)^{6}+F_{0}
$$

this results in the equation

$$
m=\frac{0.02 \times F_{b}}{(0.05 \times T)^{6}}
$$

The polynomial model was both simpler to program and much quicker to execute than either the geometric or limb darking models. The reduced execution time of the polynomial model is an important factor for a program which searches the lightcurves of thousands of stars in each field.

\section{Simulated Planetary Transit Curves}

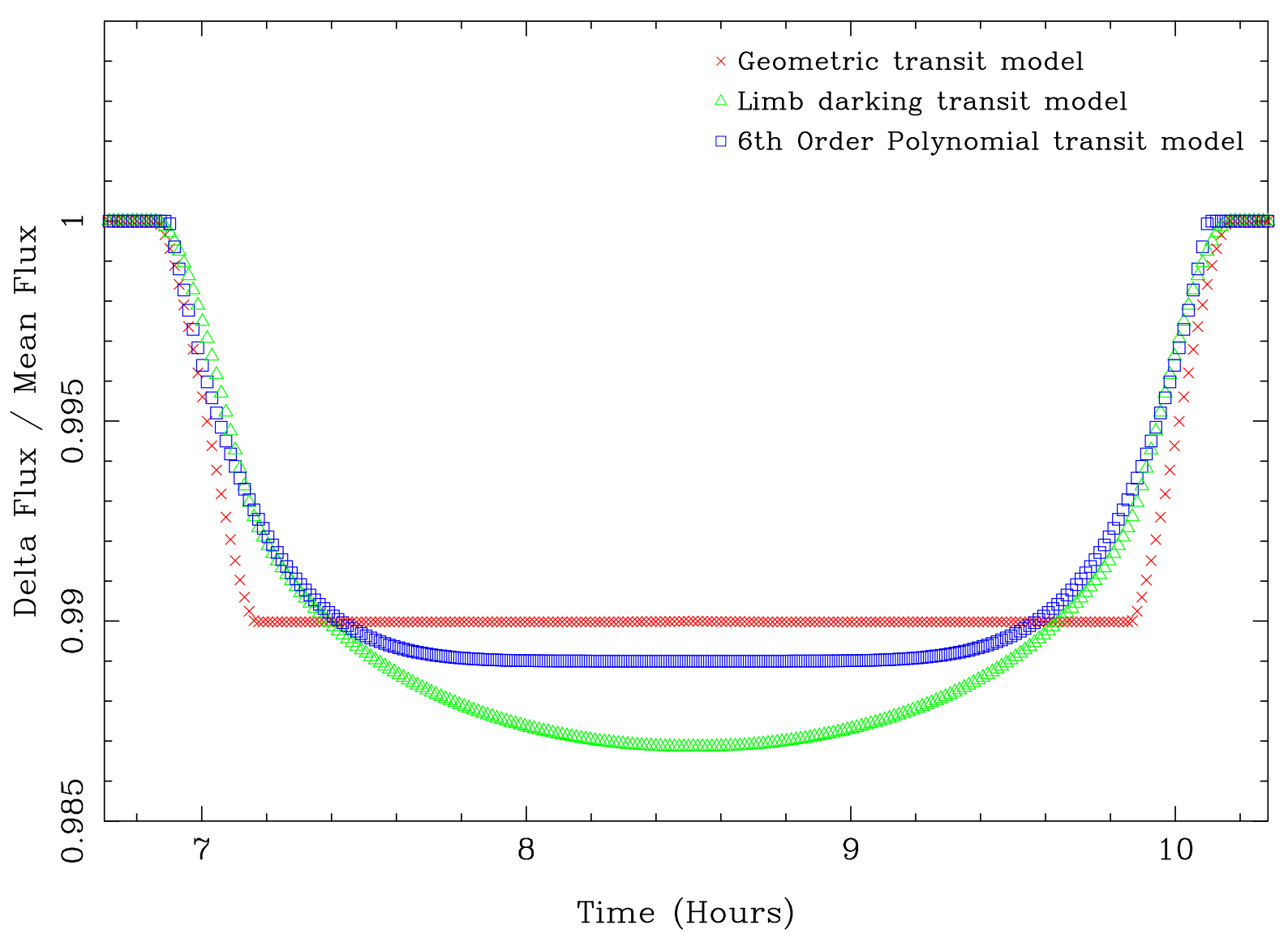

Figure 5.7: The figure shows the sixth order polynomial which was used in the detection program, compared with the geometric and limb darking transit models. 
The phase space search program was included as a function of the $\mathrm{C}^{++}$extraction program and used the mean and standard deviation values calculated in the extraction program to determine the quality of the photometry for each star. Photometry levels of less than $2 \%$ were required in order to detect planetary transits which deviate by $2 \%$ of the mean brightness of the star. Lightcurves which contained variations below this level were passed into the planetary transit search program.

\subsection{The search for planetary transits}

Since January 2001, the MOA project has conducted a search for planetary transits in the MOA star fields. The fields provide the large number of stars $\left(\sim 10^{5}\right)$ needed to search for extrasolar planetary transits, with the only limiting factor being the photometric quality of the data. It was important to analyse the MOA data for the statistical properties of brightness and time before undertaking a search for planetary transits. These properties were used to accurately simulate the MOA data (section 5.2) and determine its quality.

\subsubsection{Statistical analysis of the MOA data}

To begin with, the statistical properties of the brightness measurements were examined. It was essential to know the properties of the MOA data before designing simulations and analysis programs.
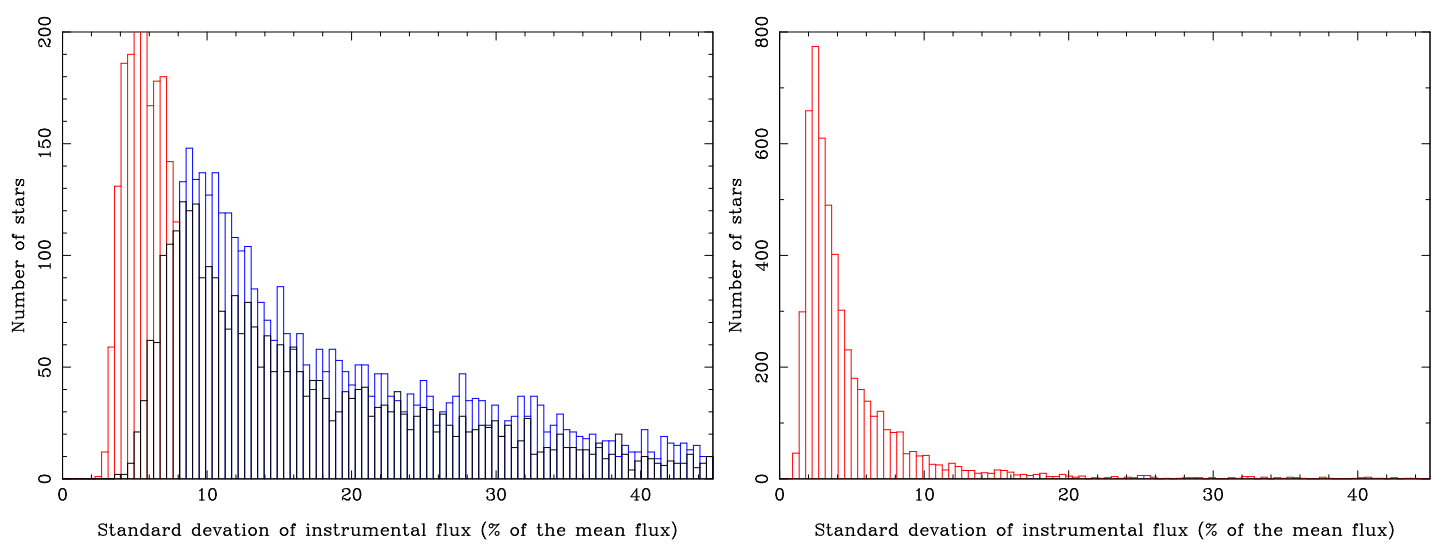

Figure 5.8: Shows the quality of the MOA-red and MOA-blue photometry for the nlmc $0-1$ (left) and the MOA-red photometry ngb $2-1$ (right) fields by plotting histograms of the standard deviation in the brightness. The histogram for the nlmc $0-1$ field shows the MOA-red photometry (red histogram), MOA-blue photometry (blue histogram) and the overlapping region of both MOA-red and MOA-blue photometry (black histogram).

Figure 5.8 shows the quality of the data for the MOA fields nlmc $0-1$ and ngb $2-1$. The nlmc 0-1 images were collected in 1997 with MOA-cam1 and were reduced using 
the DoPHOT reduction software. An approximately equal ratio of MOA-red and MOAblue filter images were collected during this time (51:49 MOA-red to MOA-blue). The standard deviation of the nlmc $0-1$ field peaks at approximately $6 \%$ for the MOA-red observations, the frequency of stars then decreases rapidly as the standard deviation increases. In contrast, the equivalent histogram for MOA-blue observations peaks at approximately $9 \%$ standard deviation and displays a less rapid decrease as the standard deviation increases. These results occurred because blue wavelength light is more susceptible to the effects of interstellar and atmospheric extinction. Hence, the MOA-red observations produce better quality photometry.

The ngb 2-1 images were collected in 2000 with MOA-cam2, and were reduced using difference imaging software; all the images were collected using the MOA-red filter. Sharply peaked about the $3 \%$ standard deviation level, the histogram decreases rapidly as the standard deviation increases. Only the brightest stars in the 2000 Galactic Bulge fields were selected for analysis, which resulted in a lower level of brightness variations.

The photometric analysis of the MOA fields revealed that searching for planetary transits in the 1997 LMC data would not be successful because of the lack of good quality $(<2 \%)$ photometry lightcurves. In contrast, analysis performed on the 2000 Galactic Bulge fields revealed that a search for planetary transits was viable, because a small percentage of stars displayed photometry levels which were better than $2 \%$.
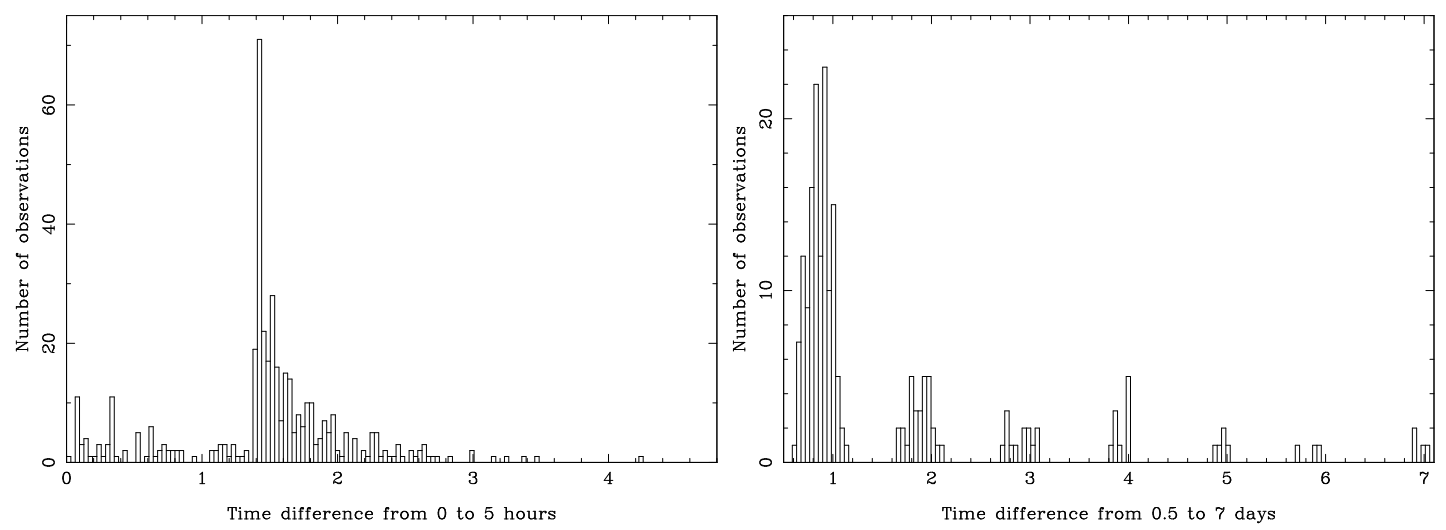

Figure 5.9: Shows two histograms of the time difference between successive observations of the MOA field ngb 2-2. The first histogram (left) shows the distribution of time differences between 0 and 5 hours, the second histogram (right) shows the distribution of time differences between 0.5 and 7 days.

A statistical analysis of the time between successive observations is shown in figure 5.9; most of the observations are separated by 1.5 hours, which is the standard amount of time needed for a complete set of galactic bulge fields to be observed and recorded. The 
histograms also reveal a significant number of observations which are separated by less than 1.5 hours; when a microlensing event that is approaching its maximum amplitude is identified within a MOA Galactic Bulge field, the MOA project stops observing all the Galactic Bulge fields and focuses solely on the single field which contains the event. During this intensive phase of observation, the images are separated by the time taken to observe and record one field (5-30 mins).

\subsubsection{Planetary transit lightcurves}

Two data reduction systems were implemented during the search of the 2000 Galactic Bulge fields. Images collected at Mount John University Observatory were reduced on site using Ian Bond's difference imaging data reduction program (Bond et al.2001). The output from difference imaging recorded the difference in observed flux relative to the reference image ('delta flux') for each of the selected stars and placed the values into database files.

I selected the top 10000 brightest (but not saturated) stars in field ngb 1 (chips 1, $2, \& 3$ each). Using this benchmark, I then selected stars in other fields which are as bright as the 10000 in other fields. I then measured the delta fluxes at these positions on the corresponding difference images. ${ }^{2}$

For each field examined in the search for planetary transits, a reference image (which was recorded under good seeing conditions) was reduced with DAOphot II, which produced the baseline brightness values stars found in the fields. Output files from DAOphot II/Allstar were cross-referenced for position with the database files produced with difference imaging. The delta flux values calculated by difference imaging were added to the baseline brightness values determined with DAOphot II, and placed into the StarBase database. Each of the fields in a StarBase database could then be accessed by the planetary transit search program.

Certain requirements were built into the search program, in order to rapidly eliminate lightcurves where planetary transits could not possibly be detected. The first requirement was that all lightcurves must contain more than a set level of 175 observations. This level was determined when the properties of planetary transits of the object HD 209458 were examined; the transits which were observed had a duration of approximately 3.25 hours and a period $P=3.52495 \pm 0.0003$ days (Jha et al. 2000). These parameters gave a transit duration which was approximately $4 \%$ of the total orbital period, thus only $4 \%$

\footnotetext{
${ }^{2}$ Ian Bond, personal correspondence, 6 April, 2001.
} 


\begin{tabular}{|l|r|r|r|}
\hline Field Name & Total Stars & Ref Stars & 2\% Stars \\
\hline \hline ngb1-red-1 & 10000 & 9724 & 35 \\
\hline ngb1-red-2 & 10000 & 10000 & 67 \\
\hline ngb1-red-3 & 10000 & 10000 & 96 \\
\hline ngb2-red-1 & 5506 & 5355 & 551 \\
\hline ngb2-red-2 & 6241 & 5956 & 771 \\
\hline ngb2-red-3 & 4044 & 3846 & 310 \\
\hline
\end{tabular}

Table 5.1: The table shows the number of candidate stars in each field, the number of stars cross referenced to find absolute flux values and the number of stars in each field with photometry better than $2 \%$.

of observations taken at random intervals would occur during a transit event. In order to show a possible transit, but differentiate it from low noise values, a minimum of seven observations needed to be obtained during a transit. A minimum of 175 observations were required to produce seven transit points, on average, with a duration of $4 \%$.

The brightest stars in each field were selected to undergo difference imaging because they show smaller variations in brightness than dimmer stars. ${ }^{3}$ Yet this selection did not discriminate between stars with low levels of variability that were needed for the search and stars with high levels of variability with noise level above $2 \%$. Consequently, the second requirement for the search program was to implement a maximum brightness standard deviation level of $2 \%$ from the mean brightness. This requirement reduced the number of potential transit curves to approximately $0.7 \%$ of the total curves averaged over all CCDs for field ngb 1 and approximately $10 \%$ of the total curves averaged over all CCDs for field ngb 2 as shown in table 5.1. Better quality image subtraction from the difference imaging reduction system for field ngb 2 meant that the quality of photometry varied substantially between fields, as evidenced in figure 5.10.

All of the stars that were passed through the first two selection criteria were then put into the phase space search program which used the maximum value of the cross correlation to find the most probable period. This value was also used to set the third requirement. Figure 5.5 shows that the cross correlation value is normally distributed about zero. A previous search for planetary transits by the large Hubble Space Telescope project, using (HST) data required the maximum cross correlation value to be $6.3 \sigma$ which gave $<1$ false alarm for the entire search space(Gilliland at el. 2000). This maximum value was based on the assumption that the noise in the HST data is close to the white noise level, however, the same assumption cannot be made about the MOA data.

\footnotetext{
${ }^{3}$ Assuming that the star does not contain some intrinsic variability.
} 

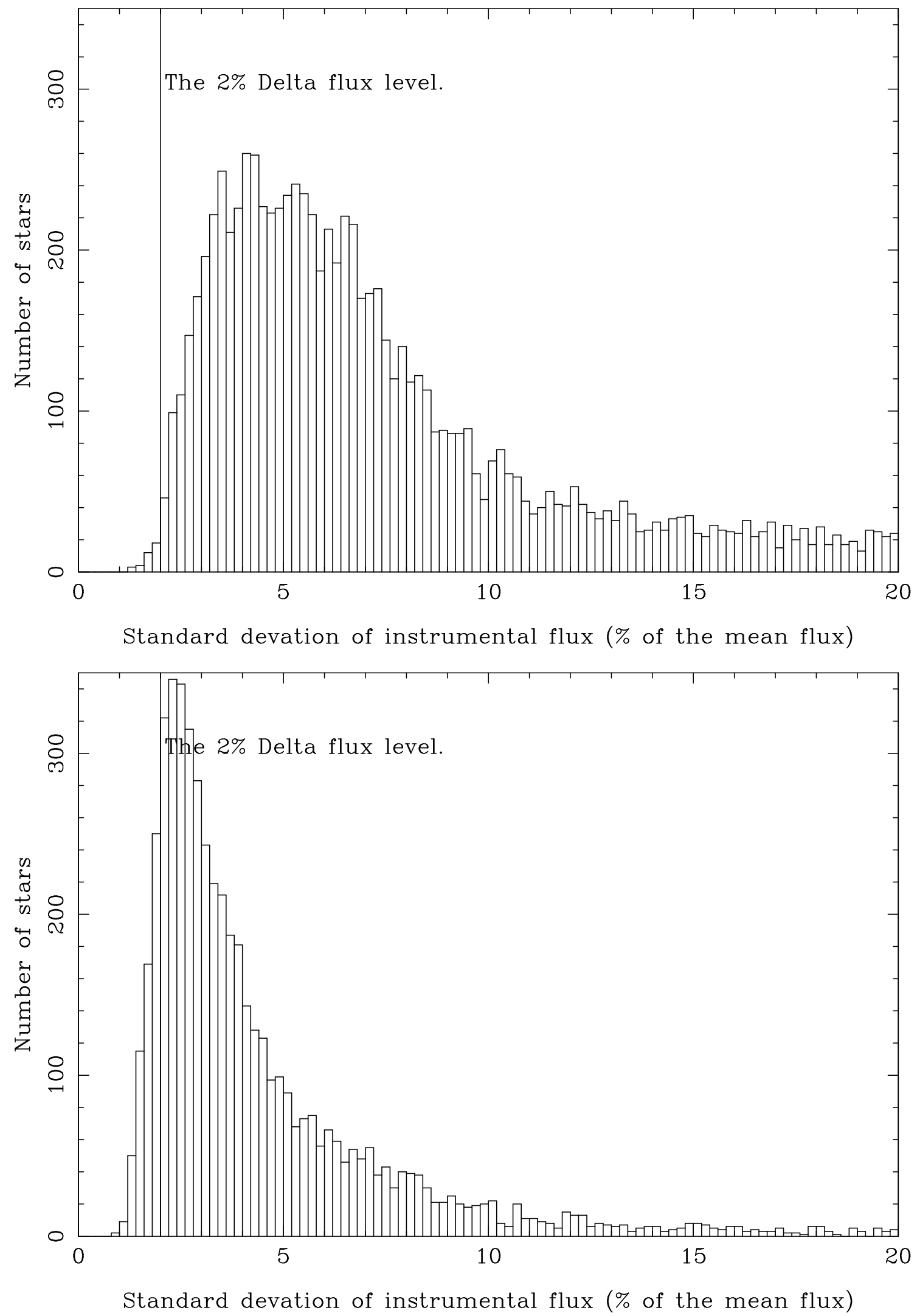

Figure 5.10: Histograms of the quality of photometry of the stars in the MOA Galactic Bulge fields ngb $1-1$ (top) and ngb $2-1$ (bottom). The quality is measured by the standard deviation as a percentage of the mean flux level for each star. 
Histogram of the Cross Correlation values

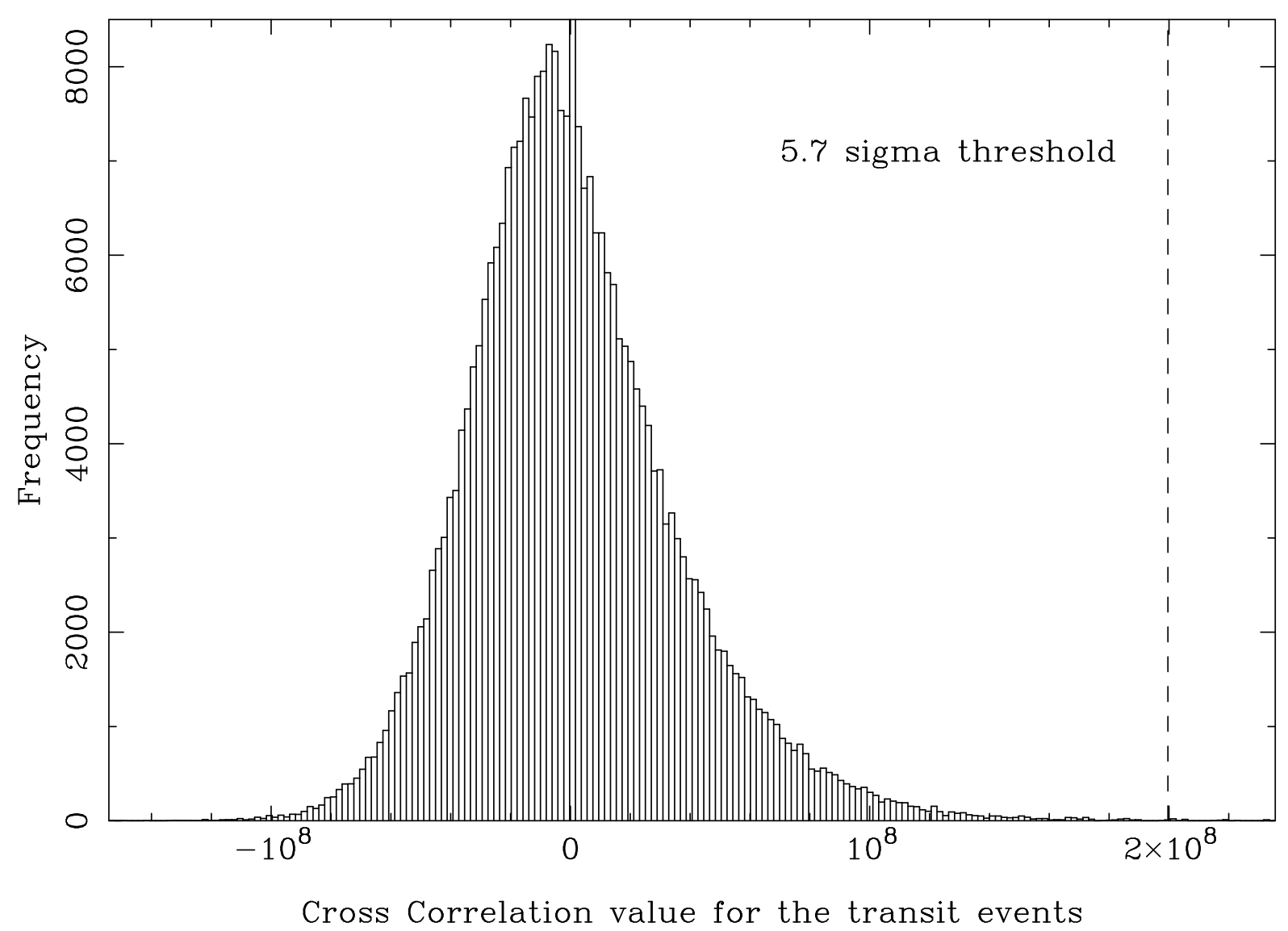

Figure 5.11: A histogram of the cross correlation values calculated for the potential transit labelled transit3156 from Galactic Bulge field ngb 1-3. The graph also shows the 5.7 $\sigma$ threshold.

The third requirement of the transit program set a lower limit for the maximum cross correlation; lightcurves that did not exceed this limit were rejected. The limit's value was $5.7 \sigma$, which was calculated using the integral probability of Gaussian statistics (Bevington 1969) and is shown in figure 5.11. Lightcurves which contained a maximum cross correlation value above $5.7 \sigma$ were selected for a more detailed search that concentrated on an area surrounding the period, which in turn had been calculated from the coarse search. The more detailed search used a $\chi^{2}$ test and the sixth order polynomial model (shown in equation 5.3) to determine the goodness of fit.

Lightcurves which met the three transit criteria were examined with periodograms of the folded data. These lightcurves were then sorted into three categories: 'not interesting', 'interesting' and 'very interesting'.

The 'very interesting' lightcurve (transit5829) was investigated further by using a transit modelling program developed by Denis Sullivan. This program fitted the limb darking 


\begin{tabular}{|l|c|}
\hline Categories & Number of lightcurves \\
\hline \hline Very Interesting & 1 \\
\hline Interesting & 15 \\
\hline Not Interesting & 79 \\
\hline
\end{tabular}

Table 5.2: The table shows the number of lightcurves in each of the transit categories.

and geometric transit models to the data and calculated $\chi^{2}$ values for the quality of the fit for both models. For an area of the folded lightcurve ranging from 4.8 to 7.2 days (see the close up section of figure 5.12), the program calculated $\chi^{2}$ values for a straight line at the mean brightness level $(\sim 16)$, the limb darking model fitted over the transit event $(\sim 10)$ and the limb darking model fitted over an area which did not include the transit event $(\sim 20)$. The parameters for the limb darking model which showed the best fit were: $t_{c}=6.0$ days, $v_{t}=5.0 R_{s} /$ day,$R_{p}=0.11$ and $b=0.55$, where $t_{c}$ is the time at the centre of the transit, $v_{t}$ is the planetary velocity across the disk of the host star in the units of stellar radii per day, $R_{p}$ is the radius of the planet relative to the radius of the host star and $b$ is the minimum distance from the centre of the disk of the host star to the path of the planet across the disk relative to the radius of the host star. Assuming that the host star has a stellar radius of $\sim 1 R_{\odot}$, the planetary radius was calculated to be $\sim 1 R_{\text {Jup }}$. Furthermore, assuming that the stellar mass was $\sim 1 M_{\odot}$ and the planetary mass was $\sim 1 M_{\text {Jup }}$ the trial period ( $T=7.4798$ days) was used to calculate the orbital radius of the planet: $D_{P}=8 R_{\odot}$.

The $\chi^{2}$ values calculated were significantly higher than expected, because the associated uncertainties for each point were not accurate representations of the scatter in the brightness for lightcurves which had been folded. The uncertainties calculated show the level of uncertainty for the conditions under which the observation was obtained. When uncertainties were compared with observations collected on the same night, they showed good correlation with the level of variation in the brightness. However, when folded together with observations obtained on different nights under different conditions, the uncertainties were not as accurate.

A finding chart was produced for the 'very interesting' transit lightcurve (figure 5.13). The object was located at the CCD coordinates $(2012,359)$ in the MOA field ngb 2-2, and corresponds to the right ascension and declination coordinates of (18:02:49.07, -30:01:58.16) ${ }^{4}$.

\footnotetext{
${ }^{4}$ equinox 2000.0 coordinates
} 


\subsubsection{Candidate lightcurves}

\section{Very interesting lightcurve category}
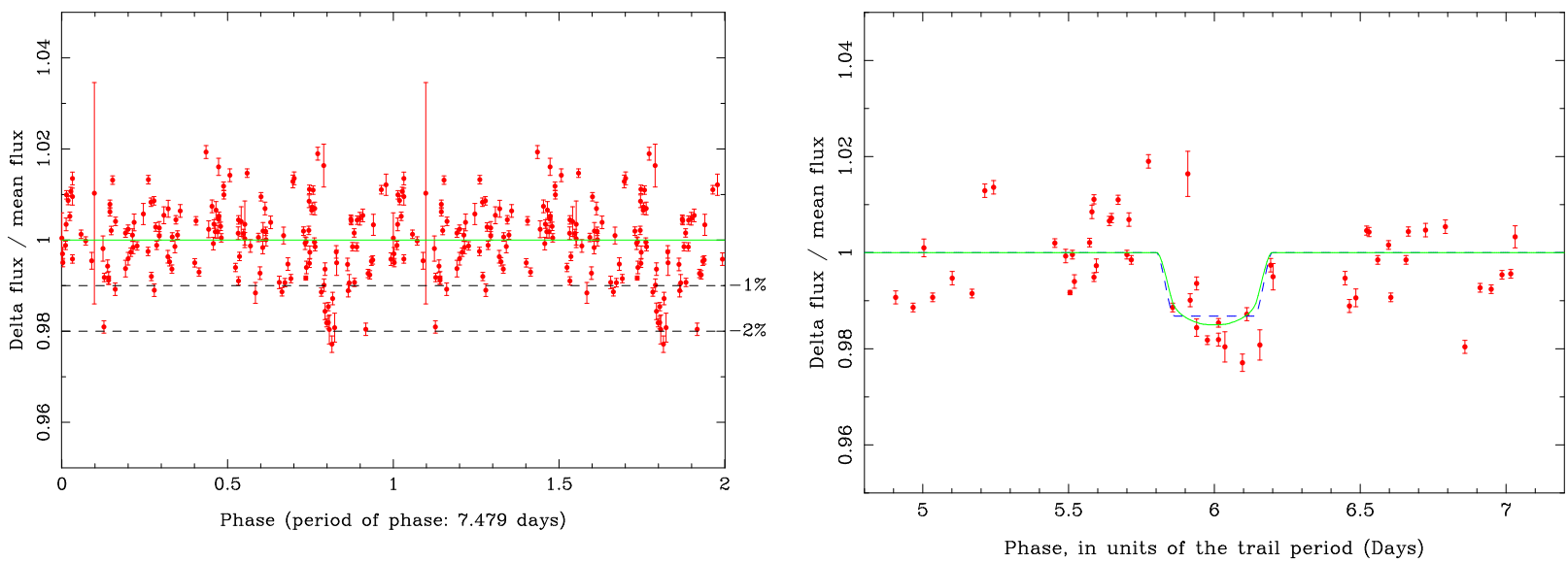

Figure 5.12: Folded lightcurves for the 'very interesting' transit curve transit5829. The close-up view of the transit shows the folded data overlaid with the best fit geometric (blue curve) and limb darking (green curve) transit models.

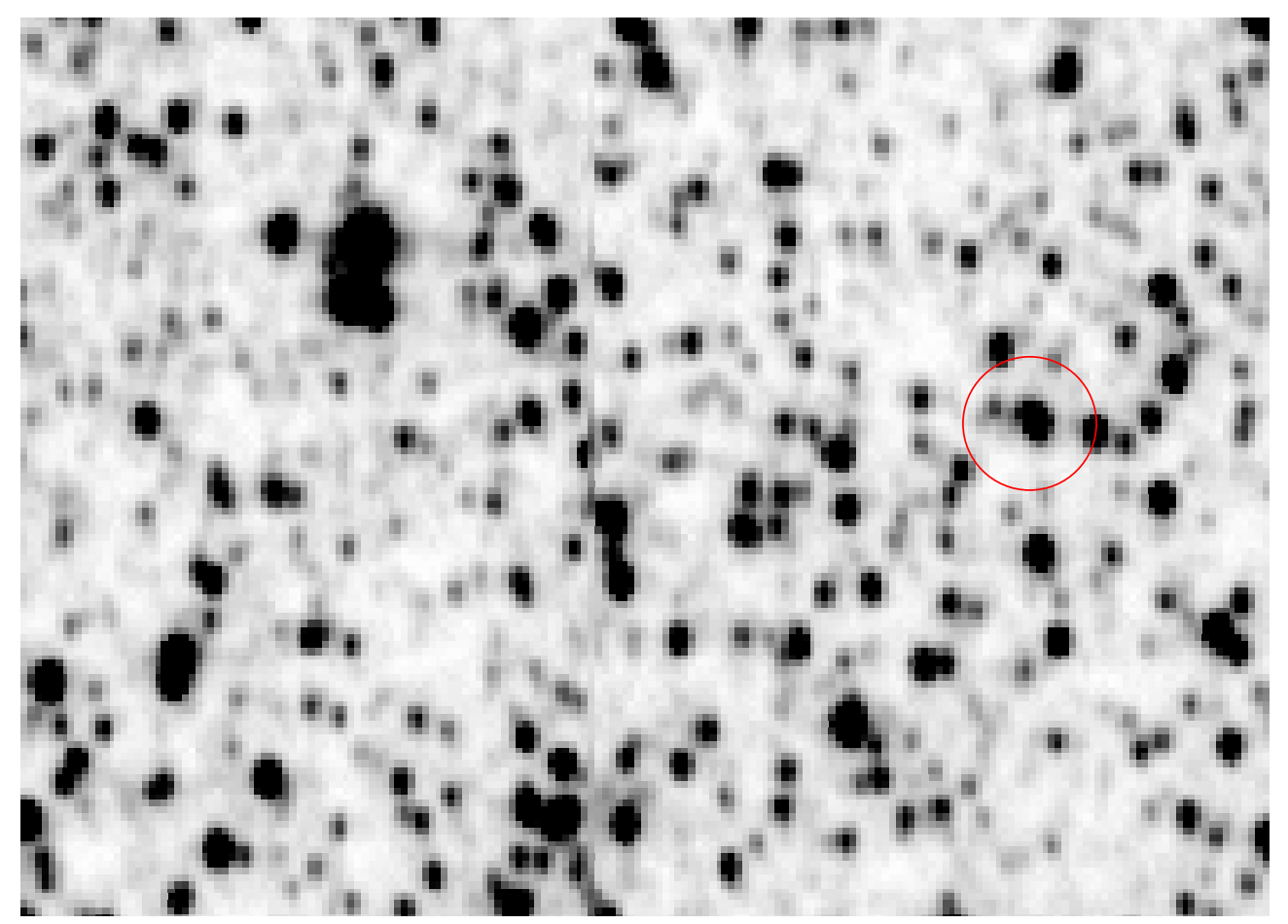

Figure 5.13: A finding chart for the 'very interesting' transit lightcurve transit5829. The chart shows the position of the object on the MOA ngb2-red-2 field's reference image. 


\section{Interesting lightcurve category}
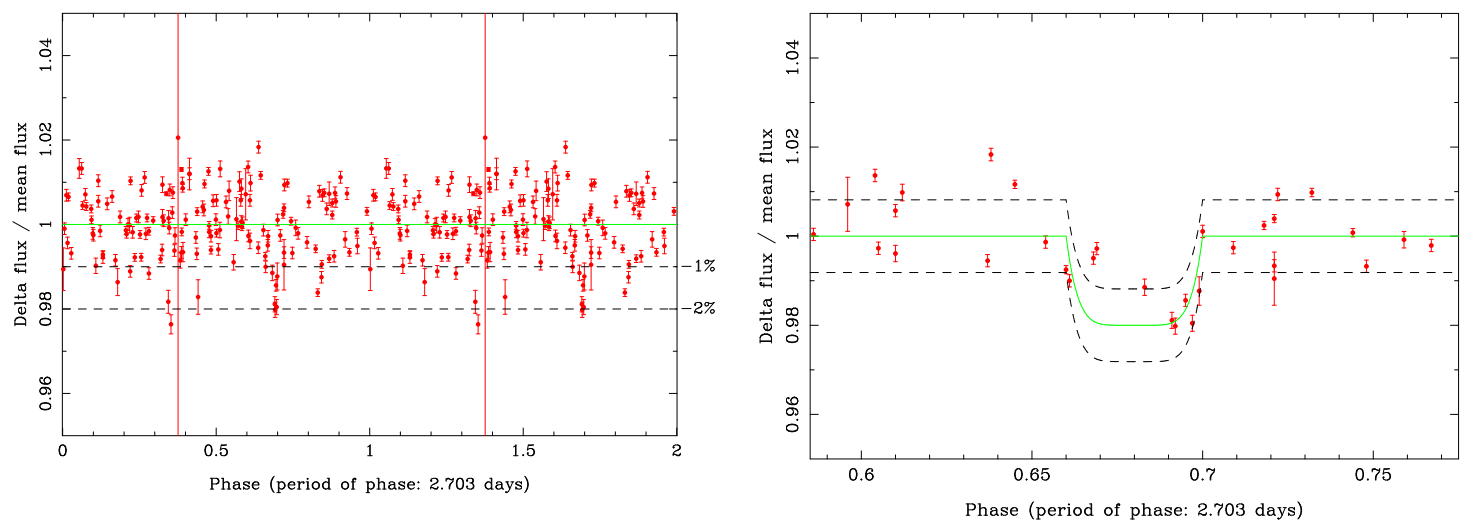

Figure 5.14: Folded lightcurves for the potential transit curve transit228. The close-up view of the transit shows the folded data overlaid with the sixth order polynomial model.
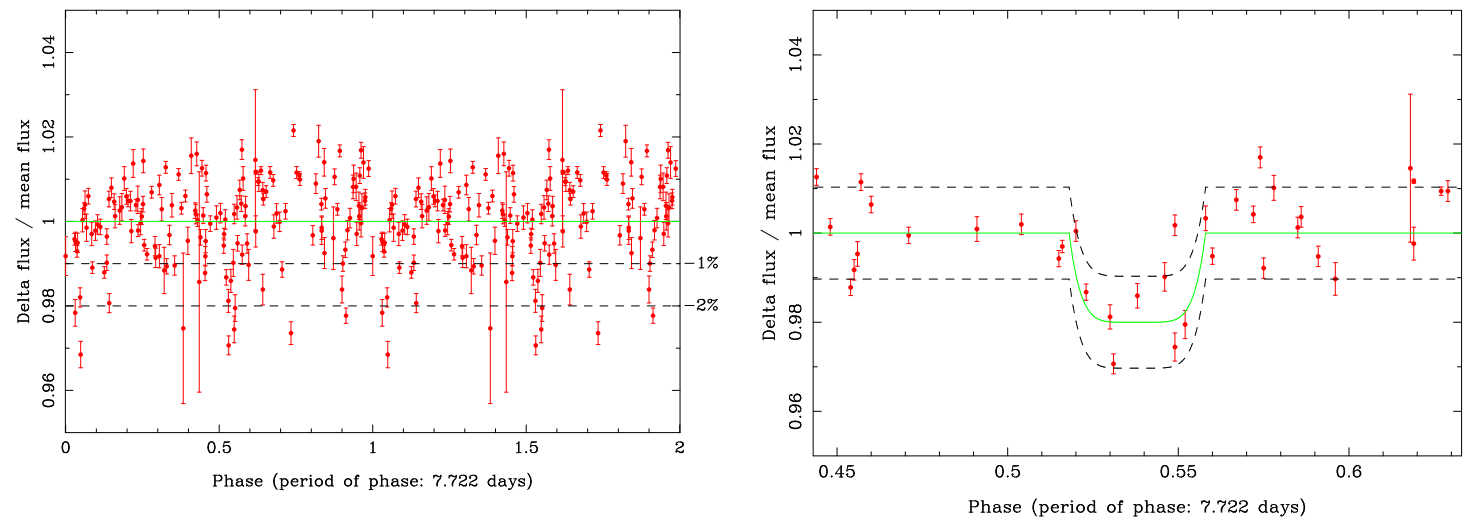

Figure 5.15: Folded lightcurves for the potential transit curve transit335. The close-up view of the transit shows the folded data overlaid with the sixth order polynomial model.
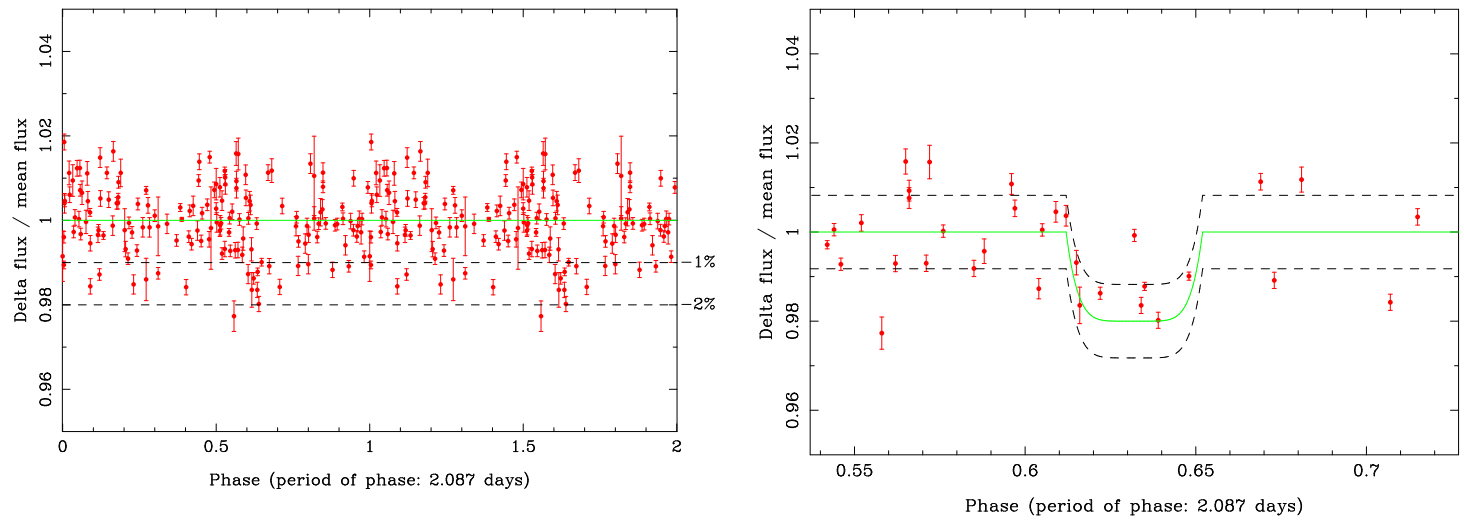

Figure 5.16: Folded lightcurves for the potential transit curve transit1337. The close-up view of the transit shows the folded data overlaid with the sixth order polynomial model. 

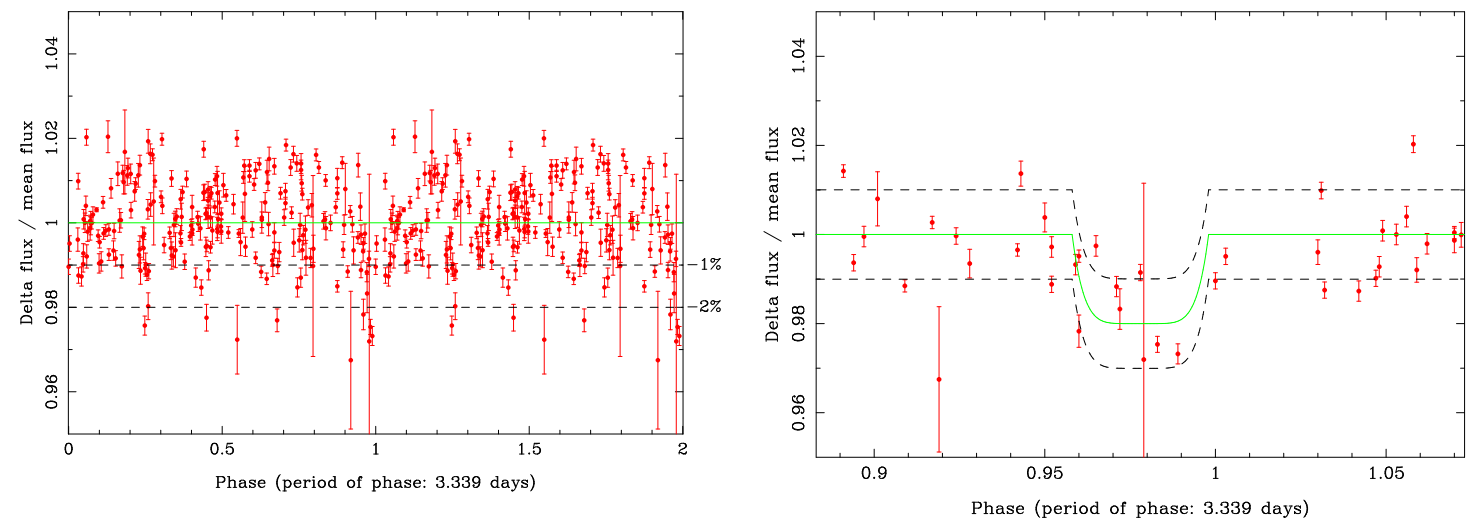

Figure 5.17: Folded lightcurves for the potential transit curve transit2722. The close-up view of the transit shows the folded data overlaid with the sixth order polynomial model.
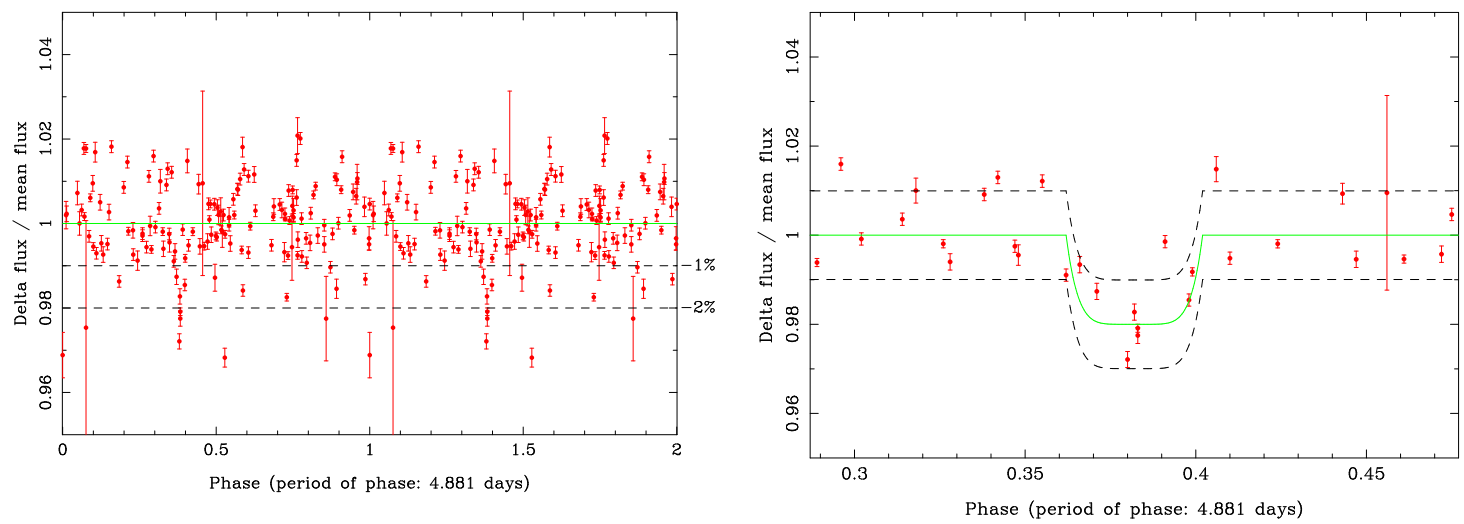

Figure 5.18: Folded lightcurves for the potential transit curve transit2859. The close-up view of the transit shows the folded data overlaid with the sixth order polynomial model.
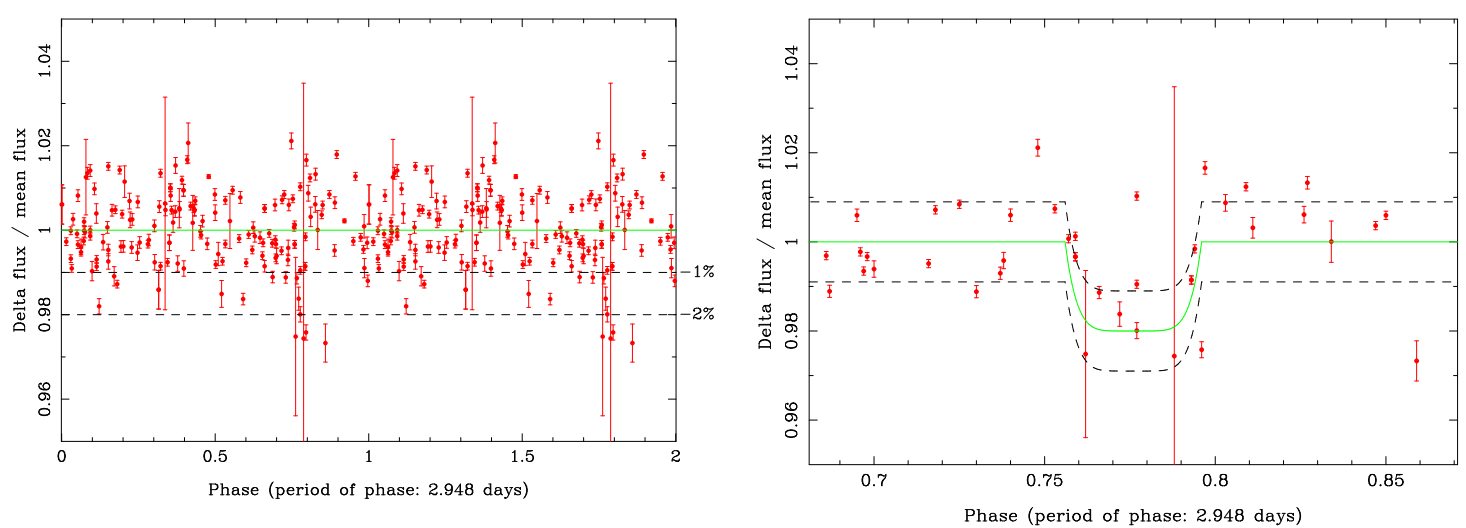

Figure 5.19: Folded lightcurves for the potential transit curve transit2949. The close-up view of the transit shows the folded data overlaid with the sixth order polynomial model. 

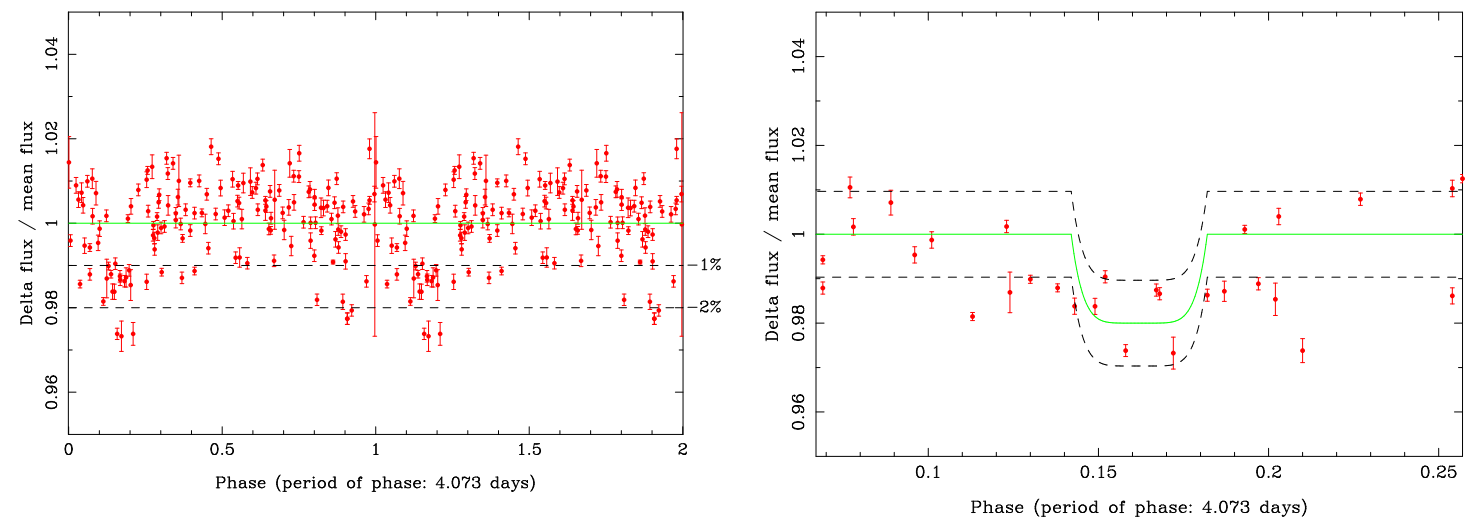

Figure 5.20: Folded lightcurves for the potential transit curve transit3156. The close-up view of the transit shows the folded data overlaid with the sixth order polynomial model.
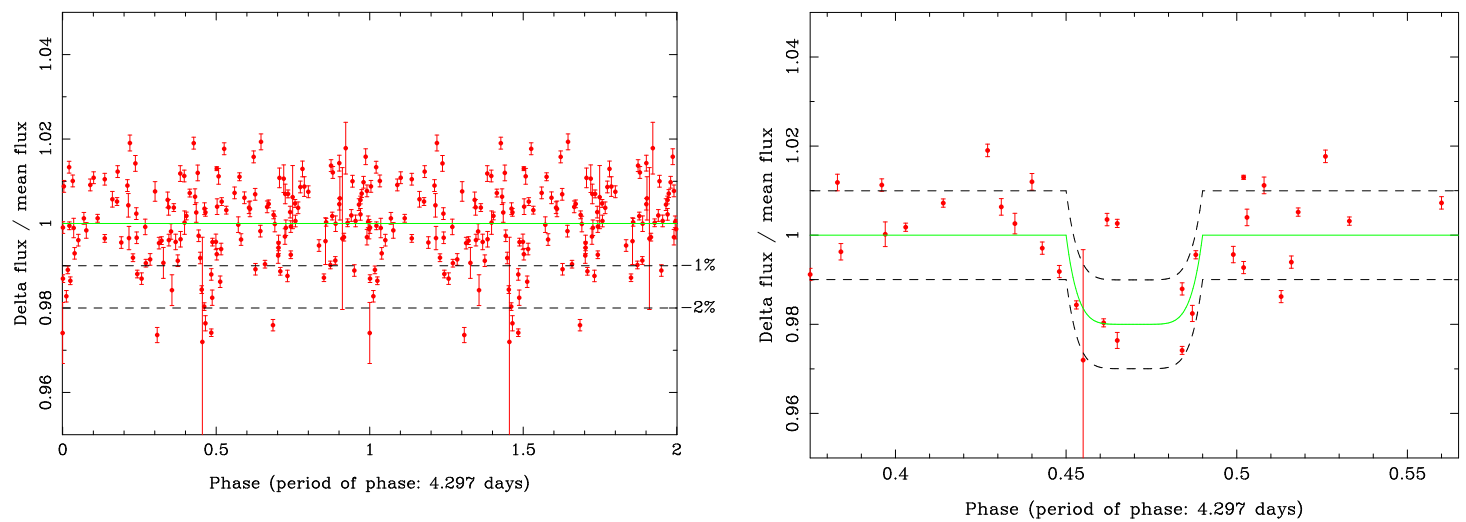

Figure 5.21: Folded lightcurves for the potential transit curve transit3175. The close-up view of the transit shows the folded data overlaid with the sixth order polynomial model.
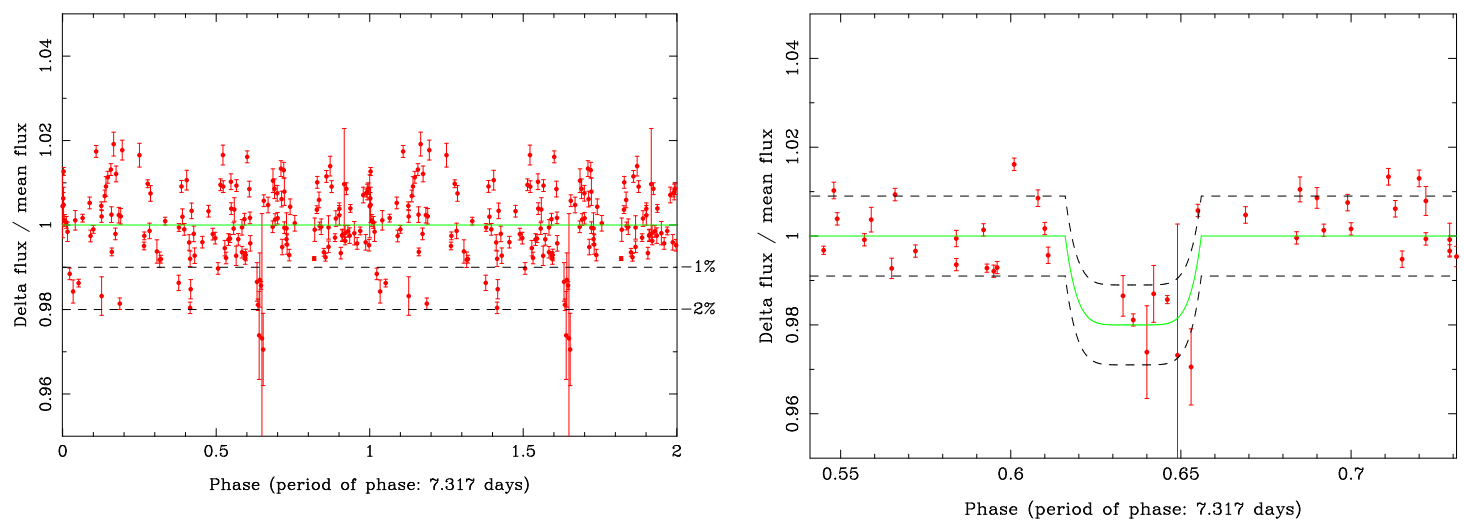

Figure 5.22: Folded lightcurves for the potential transit curve transit3816. The close-up view of the transit shows the folded data overlaid with the sixth order polynomial model. 

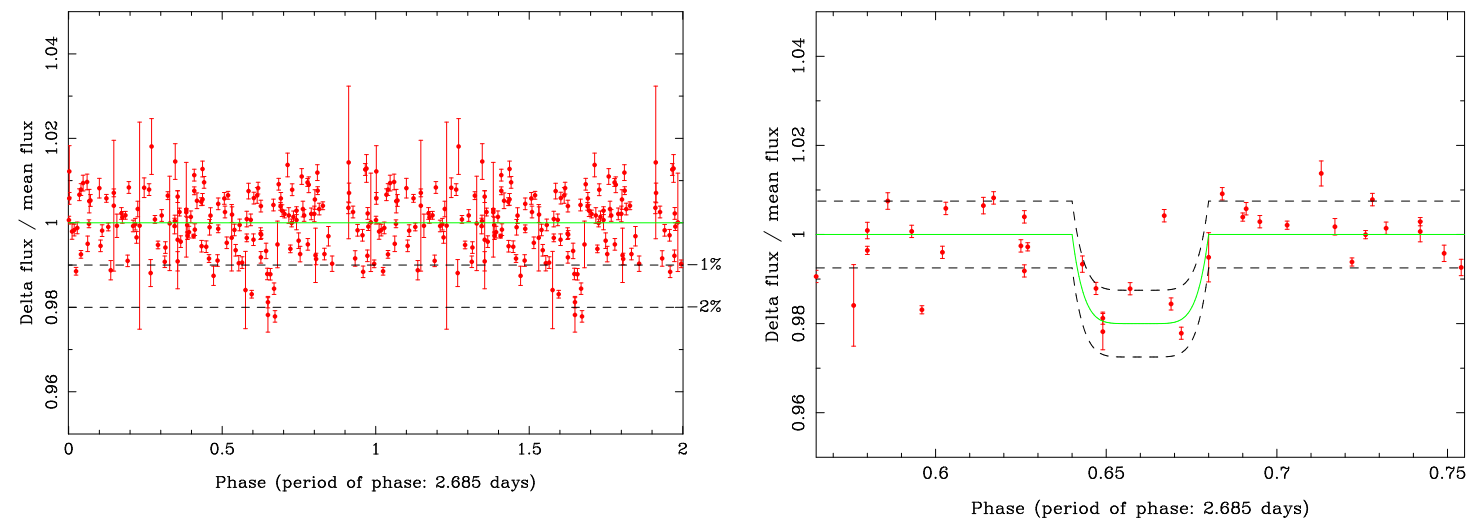

Figure 5.23: Folded lightcurves for the potential transit curve transit4567. The close-up view of the transit shows the folded data overlaid with the sixth order polynomial model.
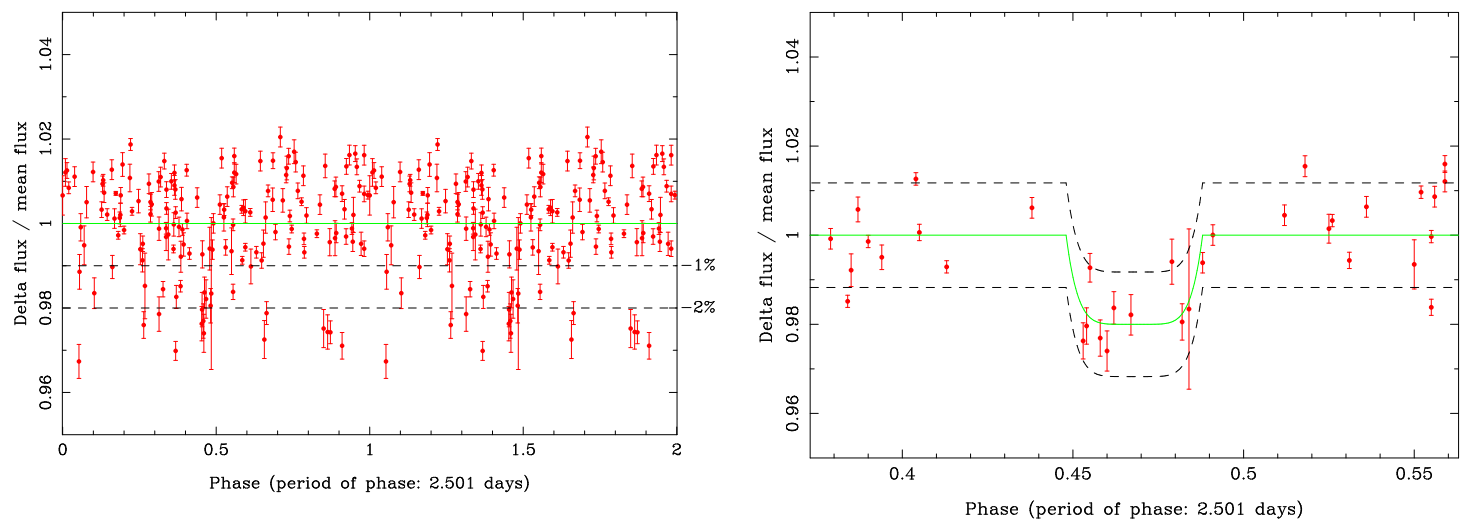

Figure 5.24: Folded lightcurves for the potential transit curve transit4741. The close-up view of the transit shows the folded data overlaid with the sixth order polynomial model.
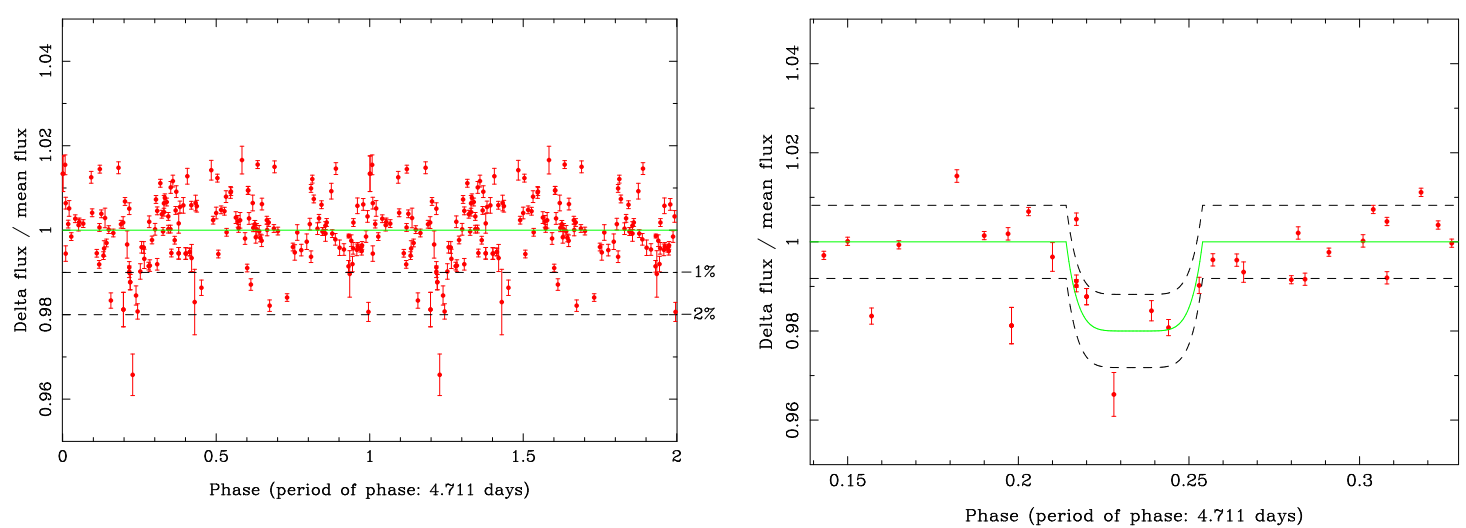

Figure 5.25: Folded lightcurves for the potential transit curve transit5479. The close-up view of the transit shows the folded data overlaid with the sixth order polynomial model. 

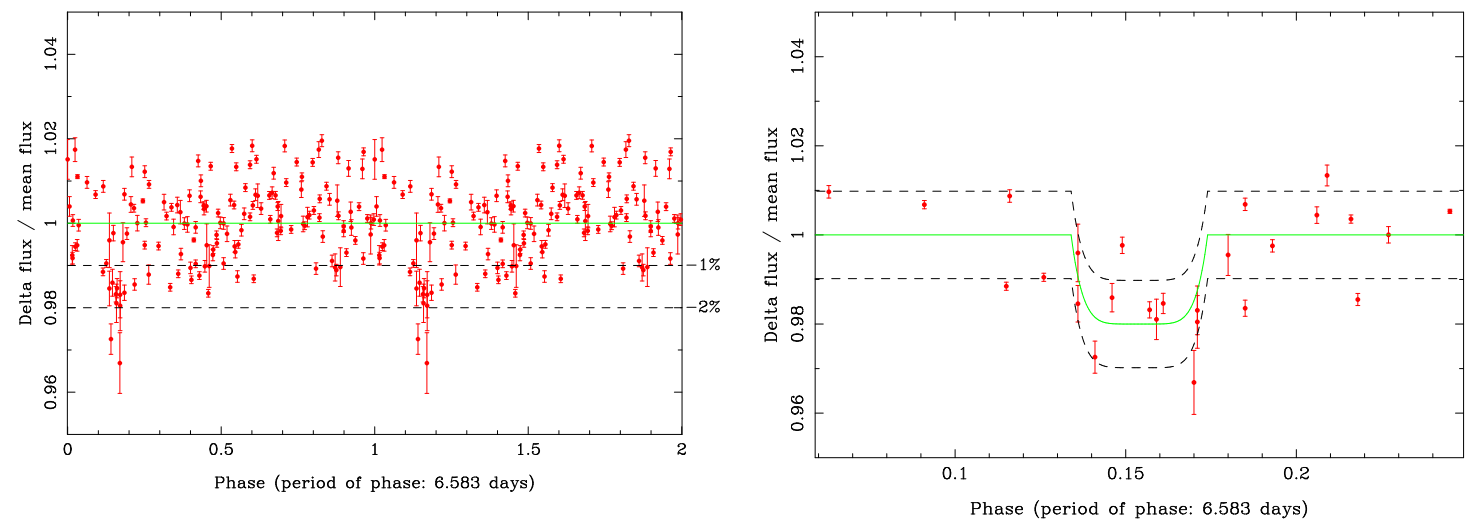

Figure 5.26: Folded lightcurves for the potential transit curve transit5610. The close-up view of the transit shows the folded data overlaid with the sixth order polynomial model.
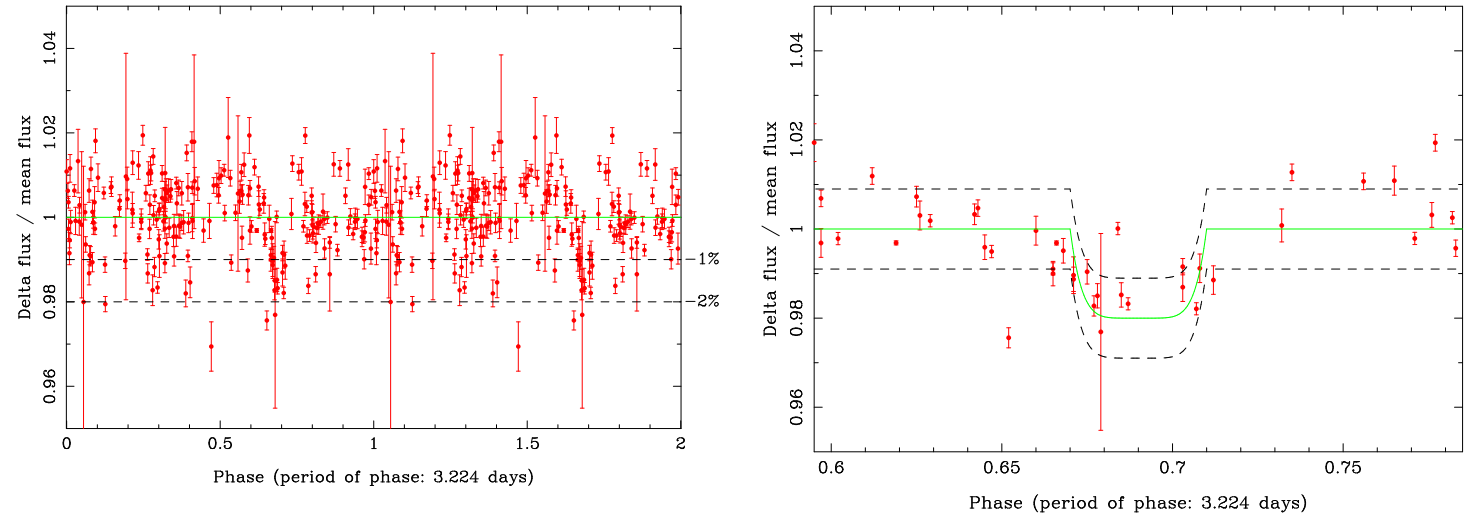

Figure 5.27: Folded lightcurves for the potential transit curve transit12414. The close-up view of the transit shows the folded data overlaid with the sixth order polynomial model.
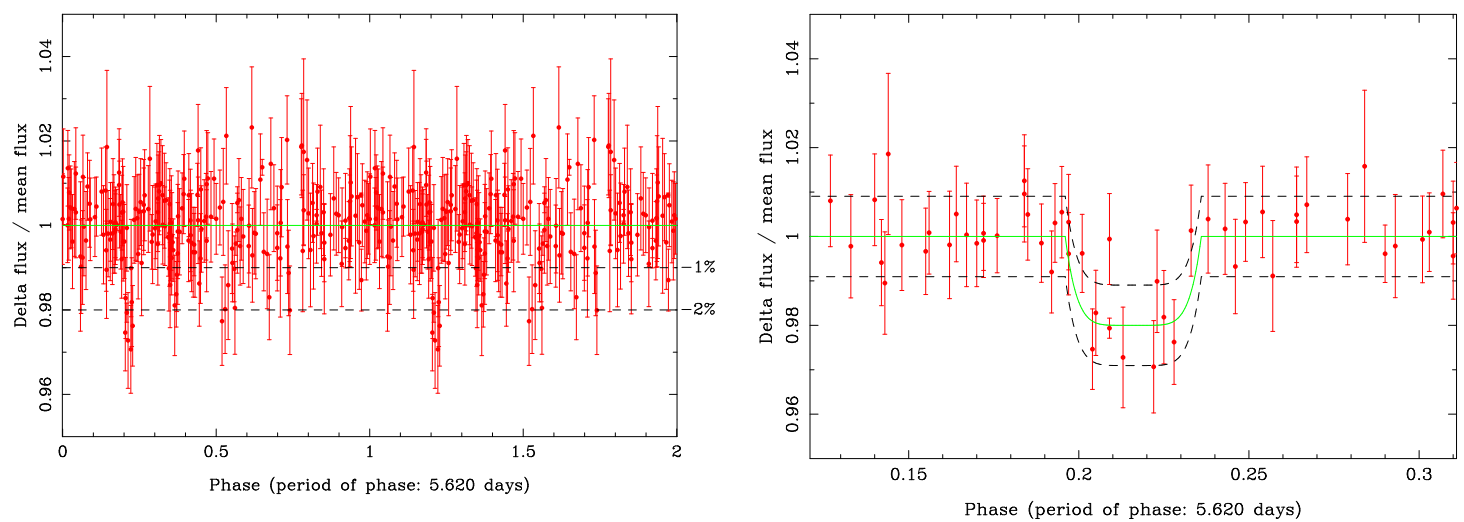

Figure 5.28: Folded lightcurves for the potential transit curve transit18269. The close-up view of the transit shows the folded data overlaid with the sixth order polynomial model. 


\subsubsection{Further analysis of planetary transit transit5829.}
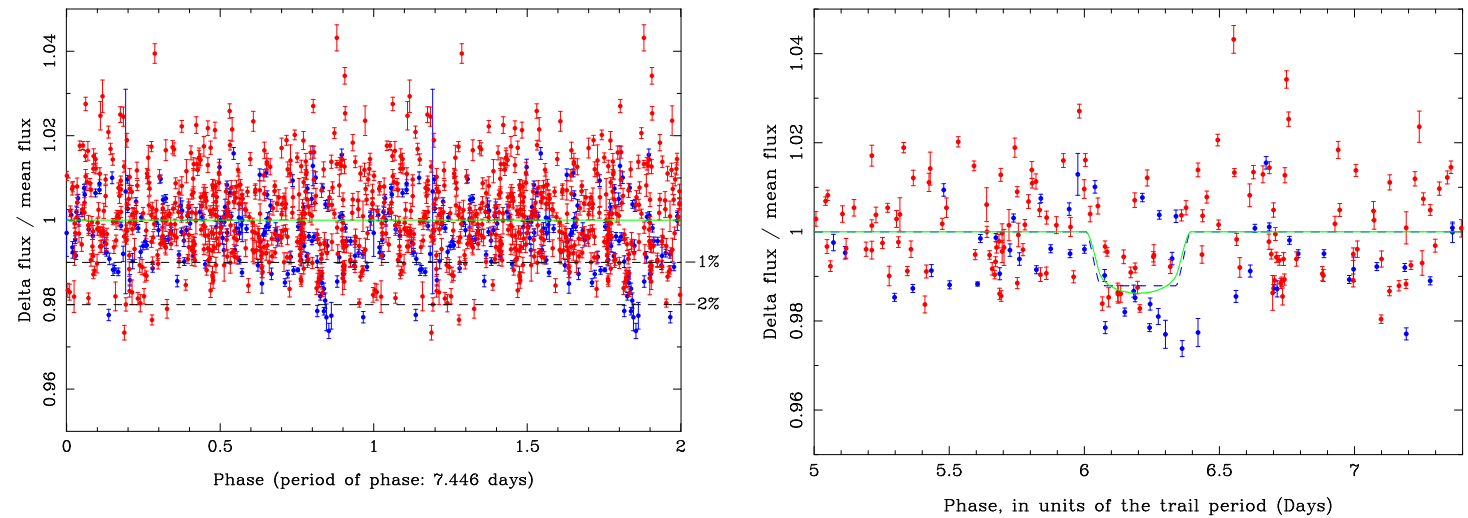

Figure 5.29: The folded lightcurve of the object transit5829, including both 2000 and 2001 data. The blue points represent the 2000 data and the red points represent the 2001 data. The search was optimised for the combined 2000 and 2001 data and produced a period of 7.4466 days. The folded lightcurve is overlaid with the best fit geometric (blue curve) and limb darking (green curve) transit models calculated for the 2000 data.

During 2001, the MOA project added a further 466 observations of the field ngb 1 to the 237 observations collected in 2000. These observations allowed for a more detailed analysis of the very interesting object transit5829. The two data sets were added together and the procedure which searches for planetary transits was repeated (described in section 5.3.2). The original search of the 2000 data calculated a period of $P=7.4798$ days. Analysis of both the 2000 and 2001 data focused on the area between seven and eight days, which revealed a period of $P=7.4466$ days (see figure 5.29).
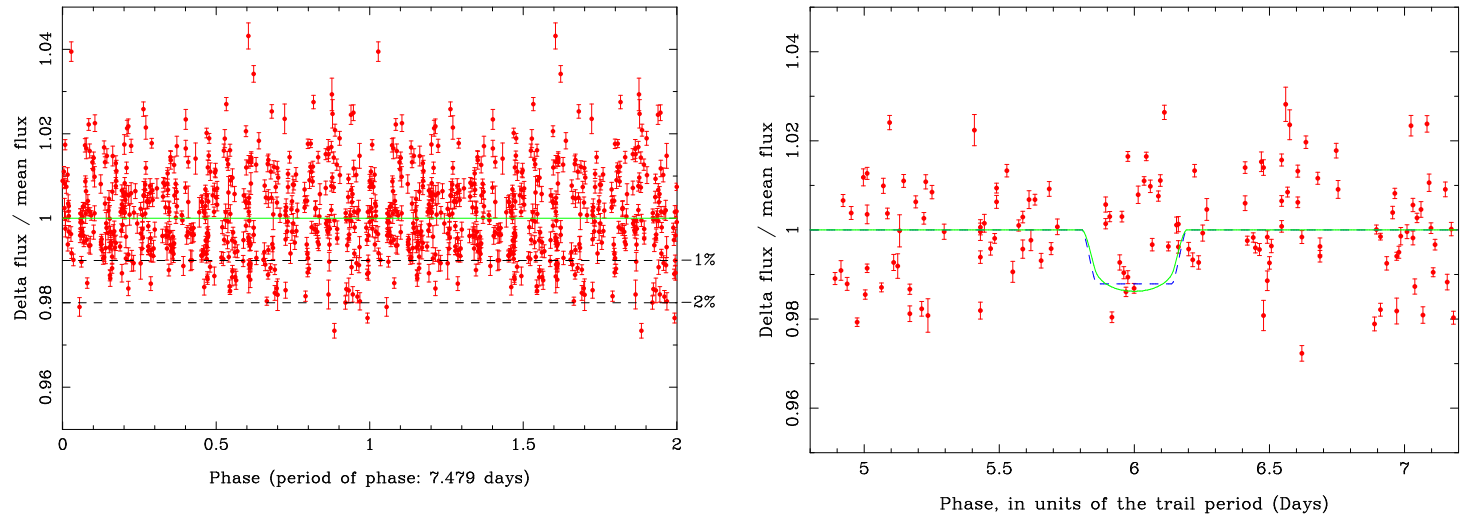

Figure 5.30: The folded lightcurve of the object transit5829, containing only 2001 data folded about the original period of 7.479 days. The folded lightcurve is overlaid with the best fit geometric (blue curve) and limb darking (green curve) transit models calculated for the 2000 data. 
A detailed examination of the 2000 and 2001 folded lightcurve suggests that the potential planetary transit was not produced by a real event. The number of observations collected during 2000 was 237, while the number of observation collected in 2001 was 466; this represents an increase of approximately 200\%. The number of observations which occurred within the modelled planetary transit was 13 in the year 2000, the equivalent number of 2001 observations was 16, an increase of $23 \%$. This relatively small increase suggests that that period of $P=7.4466$ was influenced mostly by the observations collected during the year 2000. Figure 5.30 shows the 2001 data folded around the original trial period determined for the 2000 data. The close-up view of this lightcurve shows that there is no significant deviation in the brightness of the observations from the mean level at the position of the 2000 transit. A search for transit events in the 2001 data also revealed no periodic signals with significant cross correlation values between seven and eight days. 


\section{Chapter 6}

\section{Conclusions}

The primary aim of this thesis was to detect various types of stellar variability using the extensive observational data sets of the MOA project. To perform the detection, an extraction program which worked in conjunction with the StarBase database (Reid 2002) was created. The extraction program was designed to perform searches for variable stars, microlensing events and planetary transits during a single pass of reduced images. Moreover, the program was designed so that the values required to produce colour magnitude diagrams could be calculated when star fields were observed with the two MOA filters, MOA-red and MOA-blue.

The searches for stellar variability in the 1997 MOA LMC and 2000 MOA Galactic Bulge fields were conducted with the extraction program and produced 83 Cepheid variables, 265 long period variables, 59 eclipsing binaries and 6 potential microlensing events. Sixteen potentially interesting variations that might correspond to planetary transits were detected. For the purpose of this thesis 1 folded lightcurve was categorised as a 'very interesting transit' and 15 were categorised as 'interesting transits'. Cepheid and long period variables were discovered by using a combination of the $\mathrm{C}++$ extraction program, the Welch and Stetson variability index and the Schwarzenberg-Czerny period folding program. This combination proved to be successful when searching for stars which displayed large variations in brightness. Eclipsing binaries were discovered with a modified version of the planetary transit search program, which was used after a search for variable stars of the 1997 LMC fields only produced 4 eclipsing binaries. A separate search of the 1997 LMC fields for eclipsing binaries used the modified transit program, and located 40 eclipsing binaries, while a search of the 2000 Galactic Bulge fields produced 19 eclipsing binaries. The modified transit program was selected because of the similarities between the lightcurves of eclipsing binaries and planetary transits. The modifications made to the transit program were an increase in the depth of the model transit from $2 \%$ to $15 \%$ 
of the star's mean brightness and an increase in the durations of the model transit from $4 \%$ to $10 \%$ of the trial period. A key theme of this thesis was the identification of various types of stellar variability in the MOA database. However, no attempt has been made to relate these detections to the various astronomical databases as this was felt to be outside the scope of the thesis.

To detect microlensing events in the MOA data, a microlensing modelling program that worked in conjunction with the $\mathrm{C}++$ extraction program was developed. Simulated lightcurves of the MOA data and microlensing events, which were previously discovered by Ian Bond at Mount John University Observatory, were used to test the microlensing modelling program. Lightcurves of six potential microlensing events were detected by the program. However, further examination of these lightcurves showed that the variations in brightness, modelled as microlensing events, were likely to be caused by intrinsic variability of the stars. Currently, the MOA project favours using the MOA-red filter more than the MOA-blue filter by a ratio of 9:1. This could potentially lead to false microlensing alerts, due to large brightness fluctuations produced by some types of variable stars.

In the end, the author's search for planetary transits in the MOA data sets was unsuccessful, but it did illuminate several points concerning observational procedures. In order to optimise MOA data for any future possible planetary transit searches, certain observational alterations need to be implemented, which would modify MOA's current methods of observation. These alterations are necessary in order to produce photometric precision better than $1.5 \%$ of the mean brightness of the host star. It is possible to achieve this concentrating on fields whose seeing conditions are better than 2 arcseconds. To increase the probability of observing planetary transits the MOA project would need to reduce the number of fields observed from 38 to 3 or less. ${ }^{1}$ Observations collected from the 38 fields produces less than 4 images of each field per night. To detect short duration, small amplitude variations in brightness, such as planetary transits, data points must be folded into a single trial period and this reduces the likelihood of detecting transits, because of the finite number of trial periods which can be examined. The reduction in the number of fields from 38 to 3 or less would increase the number of images per field recorded each night from less than 4 to greater than 20. Investigation of the data produced by these images could directly observe a planetary transit and use data point folding to confirm the transit and determine the orbital period of the transiting object. This increase would also improve the quality of photometry because each image would be recorded under

\footnotetext{
${ }^{1}$ The MOA project currently observes 14 fields towards the Galactic Bulge, 16 fields towards the LMC and 8 fields towards the SMC.
} 
similar conditions. It would also be practical to collect observations through two filters, as these could be used to produce colour magnitude diagrams which, in turn, could be used to select stars which belong to the main sequence of stellar evolution. It is useful to eliminate stars which belong to the red giant group, as they are less likely to reveal transiting objects of planetary size due to the substantially larger radius of the host star. If these suggestions were indeed implemented, they would decrease the probability of detecting microlensing events. Therefore, either the aims and goals of the MOA project need to be rethought, or the changes could be implemented for (say) only one observing season.

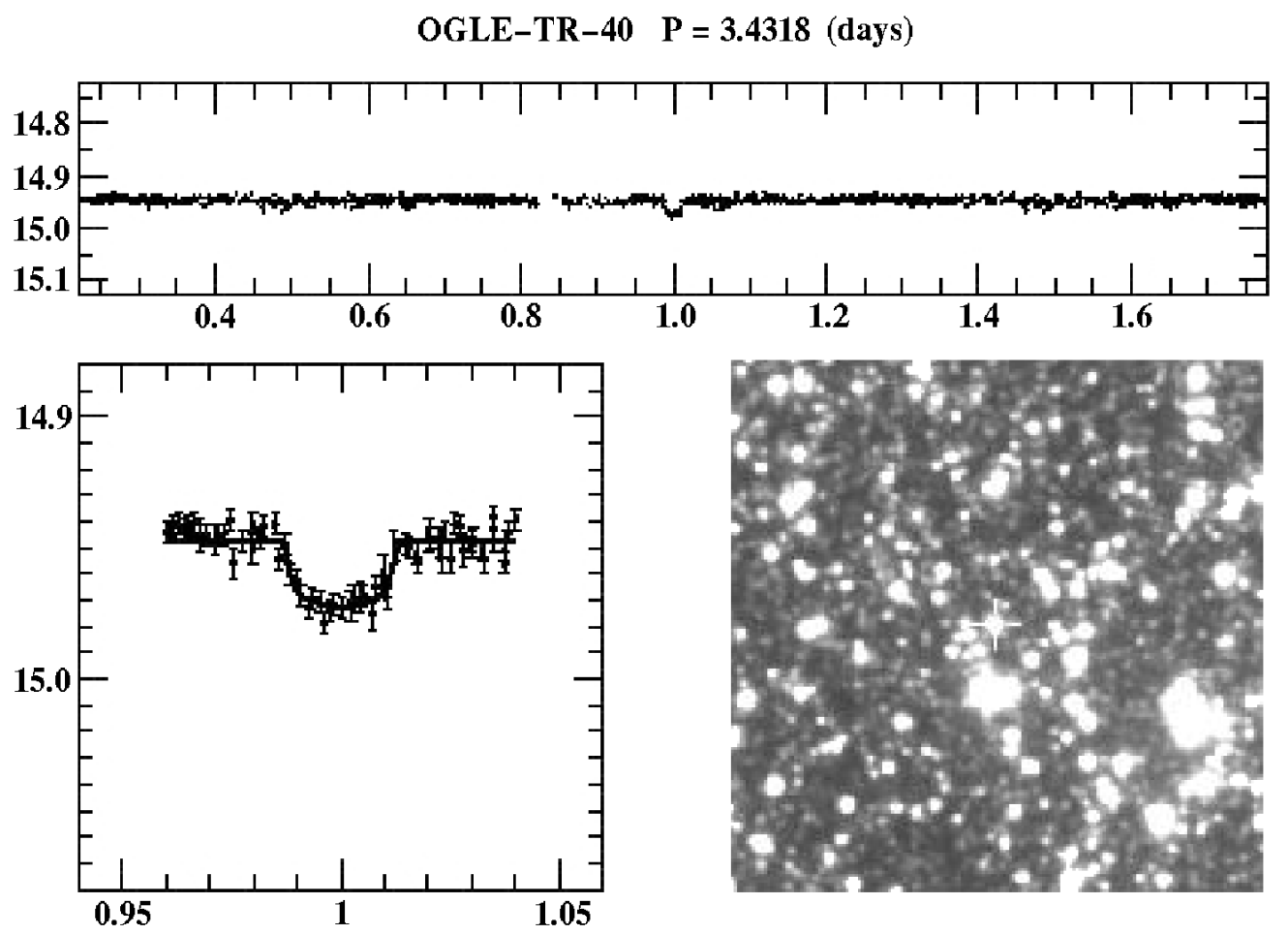

Figure 6.1: Shows close-up and extended lightcurves of a transit event for the object OGLE-TR-40 discovered by the OGLE microlensing group (Udalski et al. 2002). The figure also shows a finding chart for the object located at RA 17:57:10.27, DEC -29:15:38.1.

After this work was essentially completed, published research undertaken by the OGLE project implemented procedures similar to the ideas just outlined, during their 2001 search for planetary transits. OGLE successfully discovered events which could clearly be modelled as planetary transits (Udalski et al. 2002); their search detected 46 transit stars from 52,000 candidate objects which they observed towards the Galactic Bulge. Radius values for the transiting objects were calculated for each of the 46 objects, assuming that the mass of the host stars were all equal to one solar mass. It was calculated that eight 
objects had minimum radius values below $1.5 R_{\text {Jup }}$, a figure that is consistent with the parameters of the transiting object HD 209458, which has a calculated radius of $1.27 R_{\text {Jup }}$. Figure 6.1 shows the lightcurves and finding chart of the transiting object OGLE-TR-40, which has a radius value of $1.0 R_{\text {Jup }}$. Observations were collected with the $1.3 \mathrm{~m}$ Warsaw telescope at the Las Campanas Observatory in Chile and seeing conditions were typically 1.2 arcseconds. The quality of the seeing conditions combined with the use of difference image analysis produced lightcurves where transit events with depths "as small as 10-20 millimagnitude could routinely be detected".2 OGLE's search proved that it is possible to detect planetary transits in crowded star fields from ground-based telescopes that are operated in survey mode. In order to achieve the same degree of success from other international observational sites, it is essential to obtain high quality data, similar to that produced by OGLE.

Simulated lightcurves comprised an important part of the author's transit search program and revealed that it was indeed possible to detect transiting objects using the software. The production of accurate lightcurve simulations required detailed analysis of the statistical properties of the MOA data. These properties revealed that, despite the use of difference imaging and the selection of the brightest stars, the quality of MOA's photometric data was rarely better than $2 \%$ when the project was operating in survey mode. The MOA project also observes single fields intensively when high magnification microlensing events reach their peak. Under good seeing conditions, this method of observation produces photometric precision of approximately 1\%. High speed photometry ( $\sim 10$ second timescales) conducted at Mt John regularly achieve photometric precision approaching one part per thousand. However, the two latter methods of observation are not optimised for the detection of microlensing events and, therefore, can not be used by the MOA project. Any possible search for transiting objects undertaken by the MOA project in the future must compensate for the inferior photometry produced by the microlensing survey method at the Mt John site when compared to a site such as the Las Campanas Observatory in Chile. Although data collected at Mt John by the MOA project did not reach the photometric precision needed for a successful search for planetary transits, it has proved to be more than satisfactory in the detection of microlensing events and variable stars.

\footnotetext{
${ }^{2}$ Udalski et al. 2002.
} 


\section{Appendix A}

\section{File examples}

\section{A.1 Data reduction pipeline photometry files}

For each FITS images entered into the MOA data reduction pipeline, two files containing photometry data and other information were produced. The <image_name> out file stores the photometry of each object found in the image in the format, star identification number, x CCD pixel position, y CCD pixel position, brightness (in units of absolute magnitude), error in magnitude (in units of absolute magnitude), the background sky brightness (in units of absolute magnitude), star type, an external parameter and aperture size. The extra non-photometry related information of the image is stored in the <image_name>.info file. The \# character on the first line of the .info file is the standard comment character used for the pipeline photometry files and all files which were produced by the $\mathrm{C}++$ extraction program.

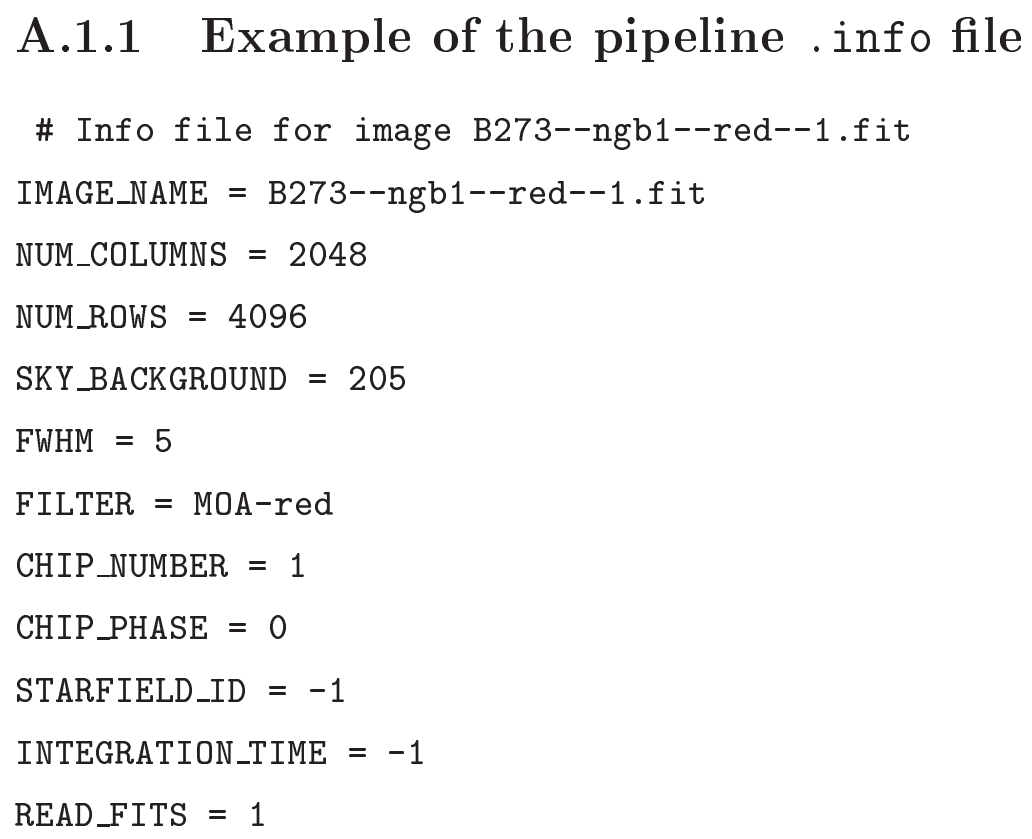




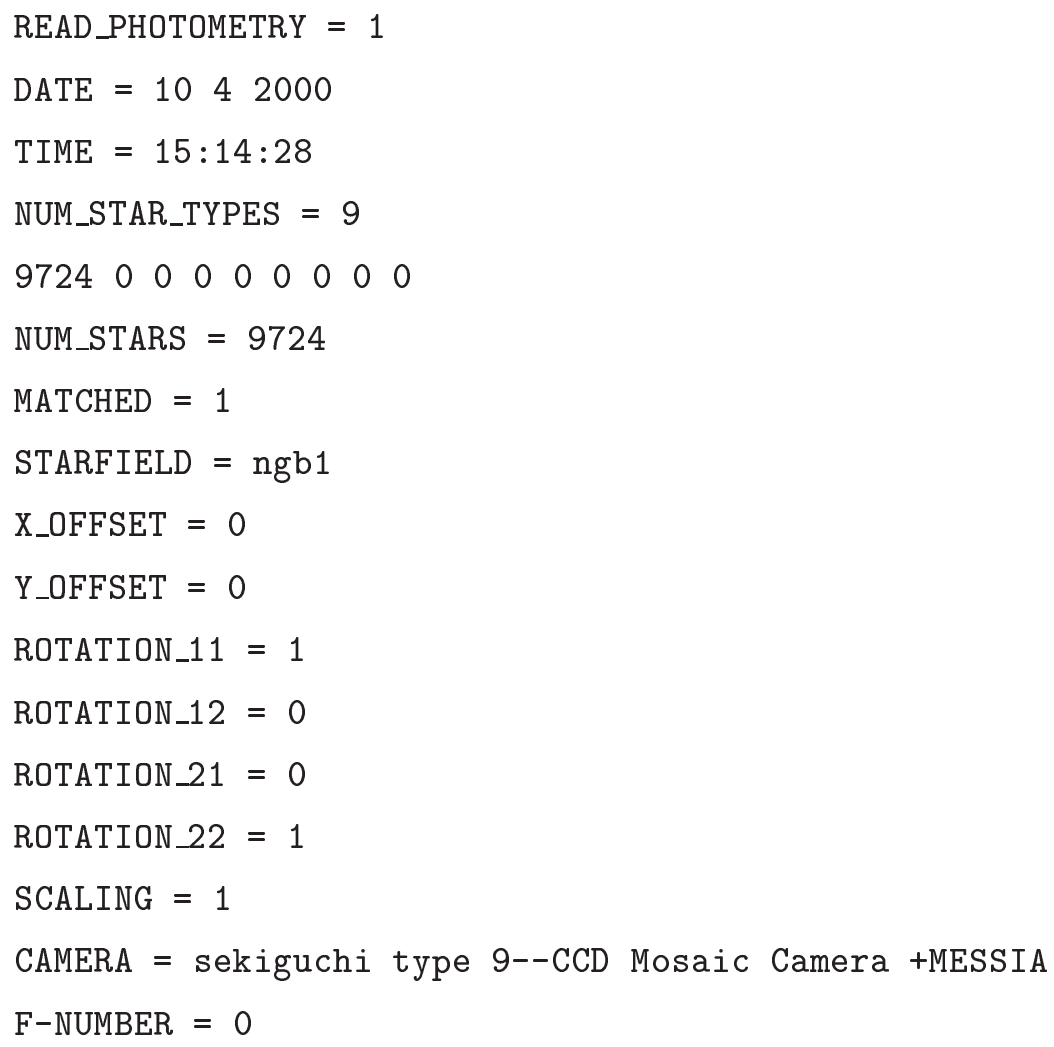




\section{A.2 Analysis files}

Data files containing photometry information for the stars recorded were produced by the extraction program and programs called by the extraction program. Colour magnitude diagrams were produced using data files created in the database extraction program and stored brightness and colour information for each star recorded.

\section{A.2.1 Example of the colour-magnitude data file}

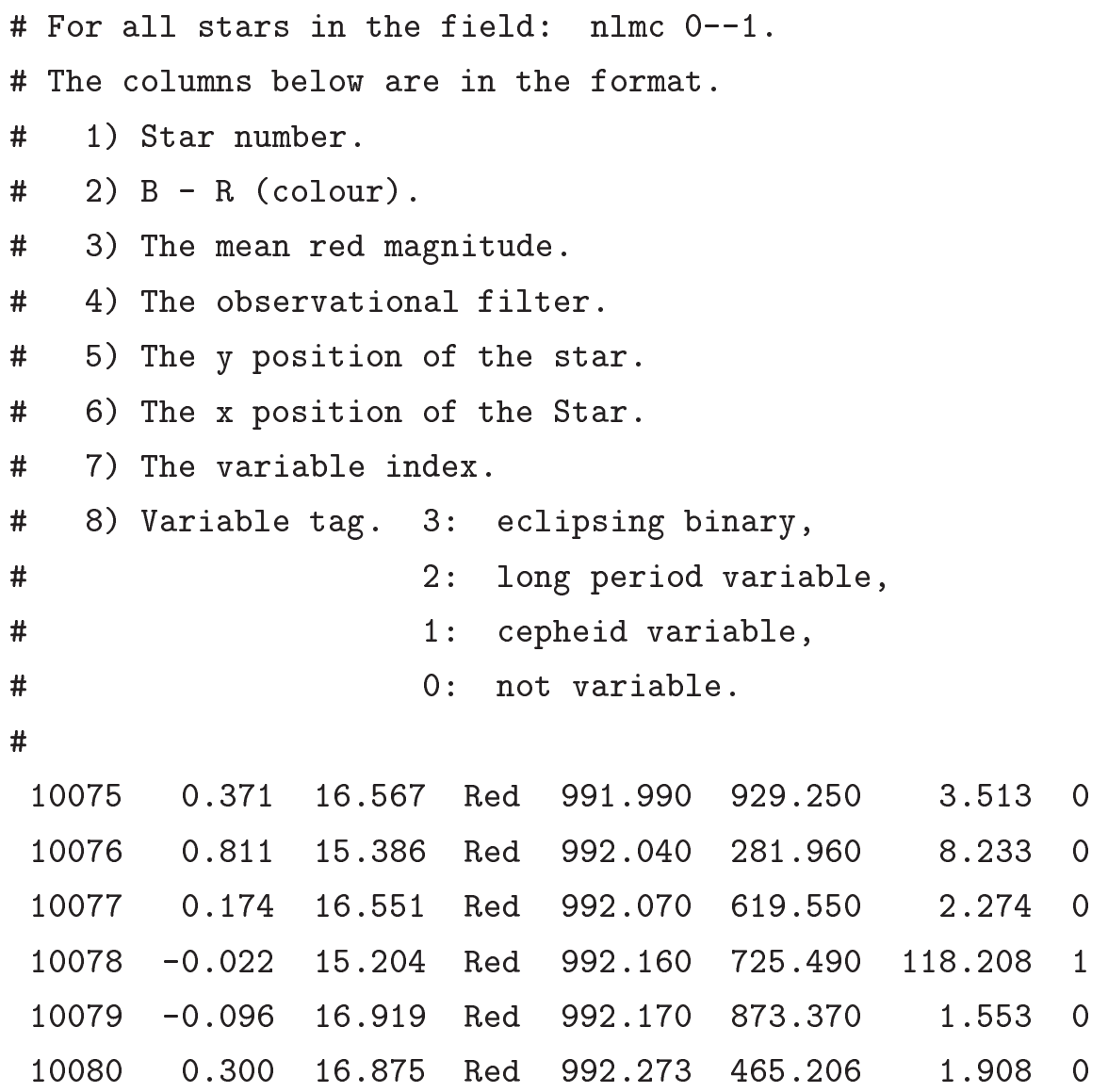

The most common format for data files produced during analysis was the photometry files which contained data to be displayed as lightcurves. A lightcurve shows the variations in the brightness of a star as a function of time. To display lightcurves which contain periodic signals the folded lightcurve was used. The folded lightcurve file has an additional column containing the phase. Both the search for period variable stars and planetary transits produced folded lightcurves for analysis. 


\section{A.2.2 Example of a folded lightcurve file}

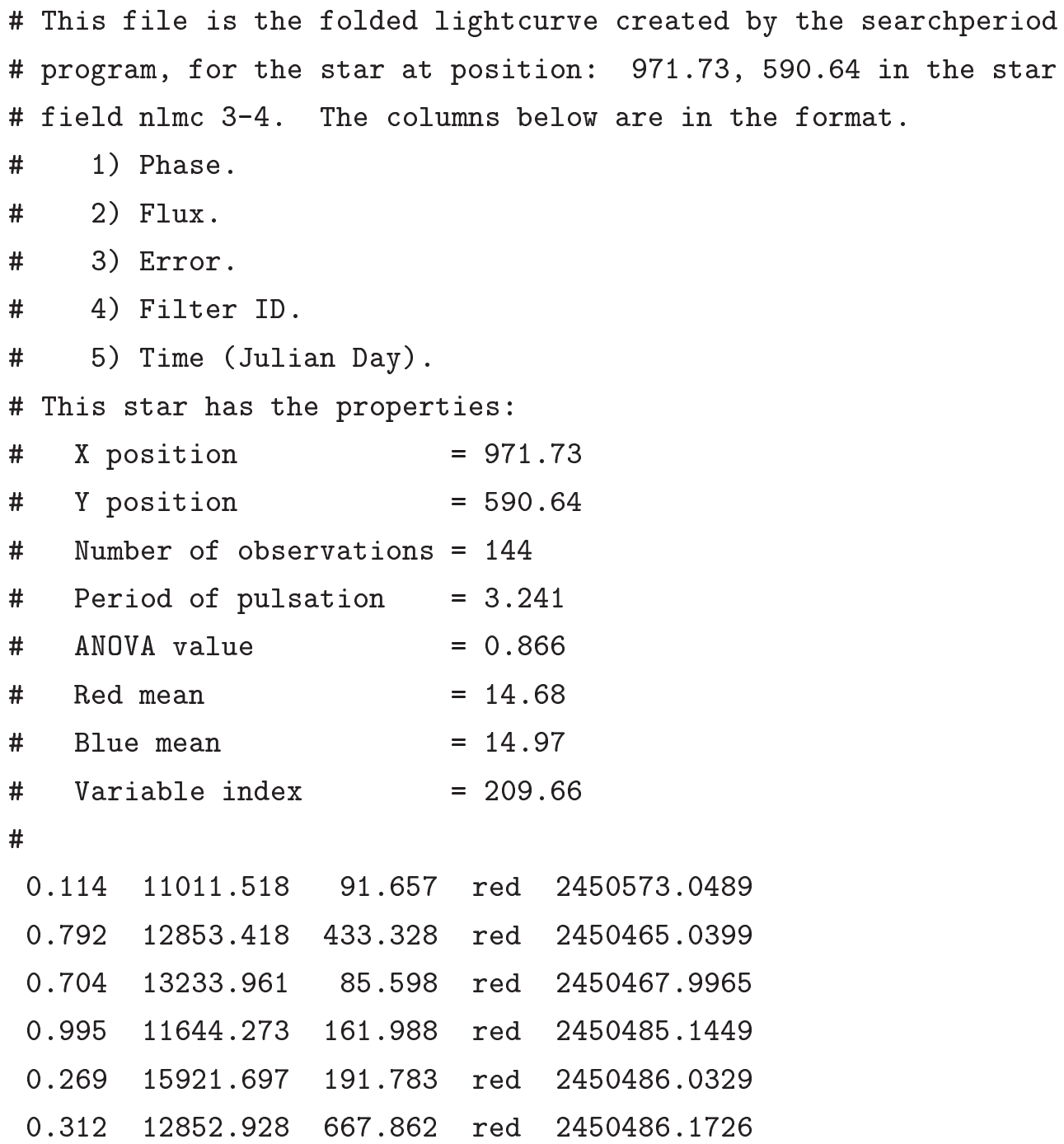




\section{Appendix B}

\section{Program structure}

The database extraction programs which were utilised during the search of the MOA database were the search.cpp and searchpos.cpp programs written by the author. The search.cpp program analyses all the objects recorded in the database of one field, while the searchpos.cpp program provides an analysis of a single object at a given position on the field. Although the search.cpp program requires no arguments, the user is prompted for information such as the field name and the type of analysis to include. Figure B.1 shows the structure of the search . cpp program. Lightcurves from the periodic variable search were plotted before the colour magnitude diagrams were produced, which subsequently allowed the position of the variables to be displayed on the diagram. The lightcurves were also plotted after all the objects in the database had been analysed, so that the program could run through the field without interruption. The searchpos.cpp program uses the 6 arguments

$-\mathrm{x}$ The $\mathrm{x}$ coordinate position of the object in the field.

-y The y coordinate position of the object in the field.

-a The minimum value of the period in the search for periodic variables, in units of days $($ default value $=1.0)$.

-b The maximum value of the period in the search for periodic variables, in units of days (default value $=50.0$ ).

-p The accuracy of the search (default value $=0.01)$.

-f The value of the filter used in the search for period variables, where $101=$ MOA-Red and $102=$ MOA-blue (default filter 101(MOA-red)). 
The searchpos.cpp program requires the $\mathrm{x}$ and $\mathrm{y}$ position arguments, however, the four subsequent arguments are not required. If any of these four arguments are not supplied by the user the default values are used by the program. 


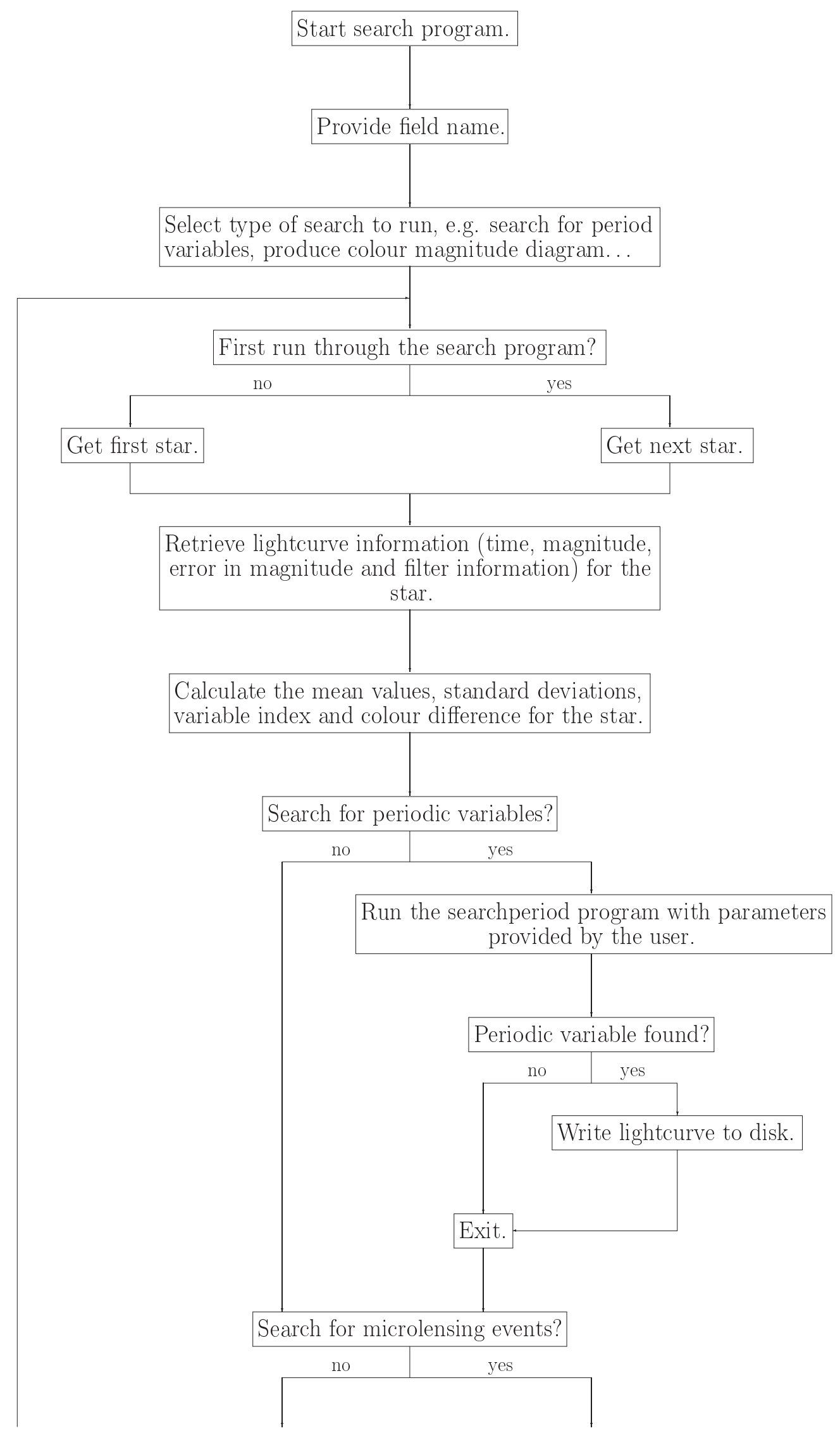




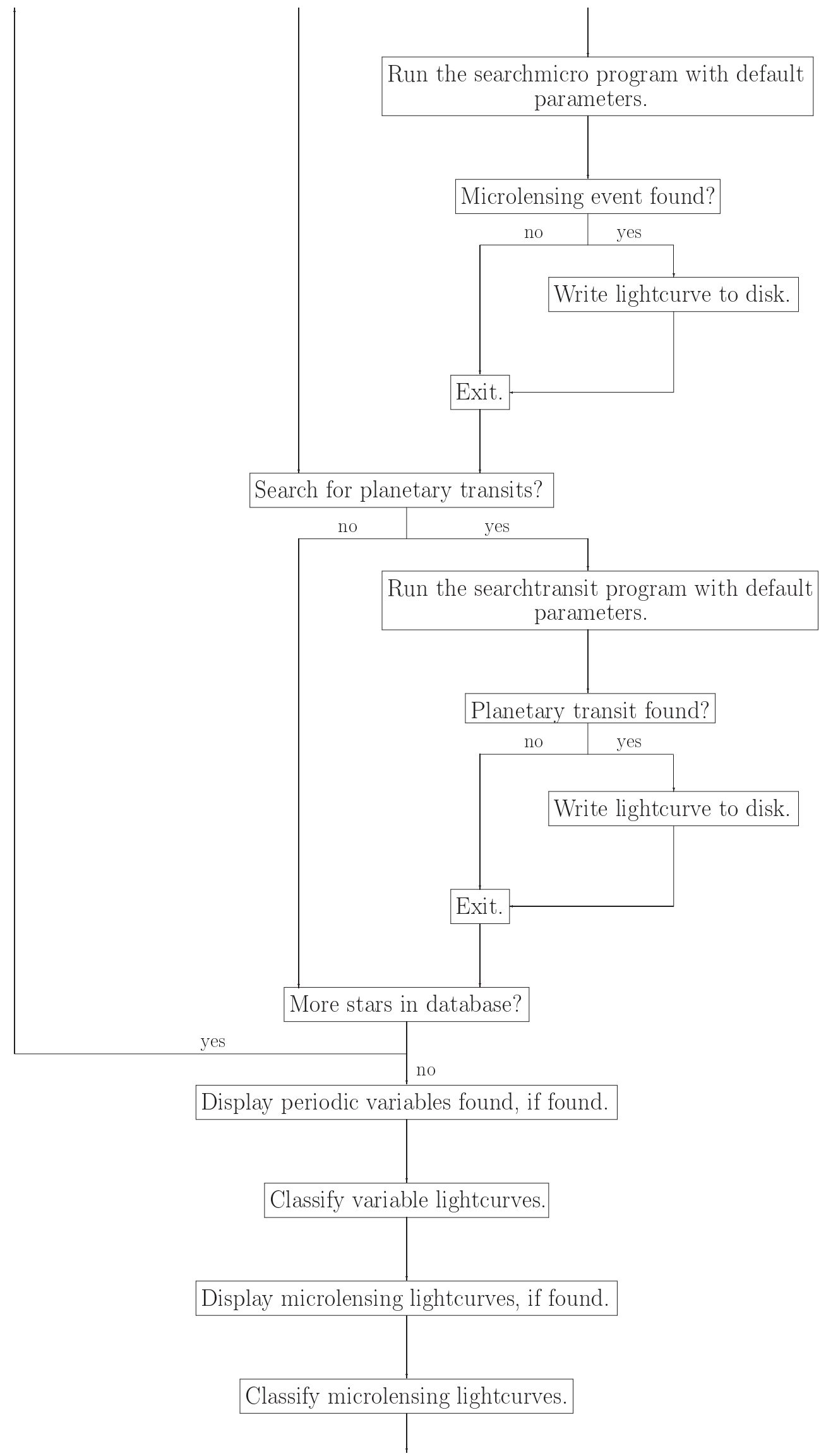




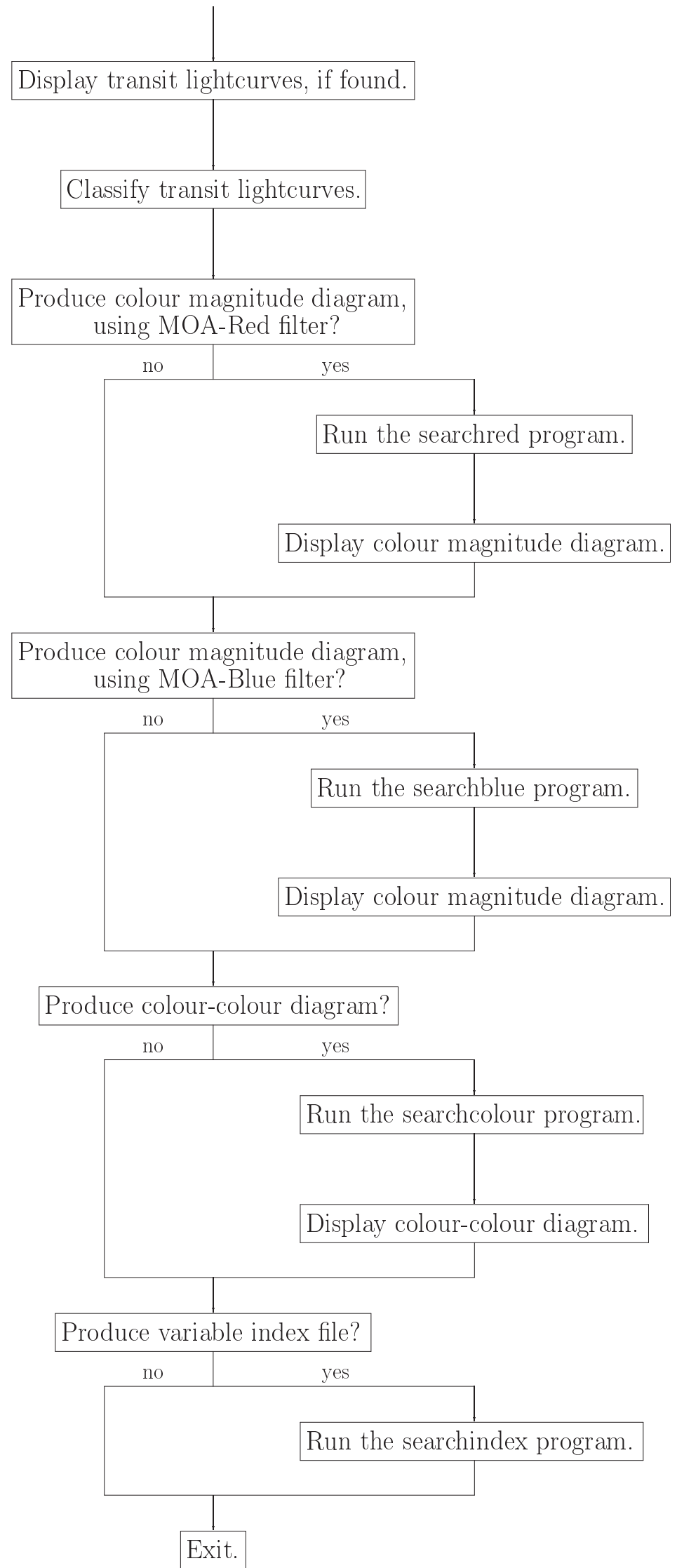

Figure B.1: Flow diagram of the structure of the search.cpp database extraction programs. 


\section{Appendix C}

\section{Catalogue}

The catalogue includes the properties of all Cepheid variables, eclipsing binaries and planetary transits found during the analysis of stellar variability in the MOA database. Each object is listed by the name allocated to the lightcurve by the $\mathrm{C}^{++}$extraction program. This name was determined by the type of variability displayed by the object and the running number of the lightcurve which was also supplied by the extraction program. The properties listed for each object were, the field origin (using the standard MOA field names), the right ascension (RA) and declination (DEC) (equinox 2000.0 coordinates), the mean brightness of the object (in units of apparent magnitude) observed with the MOA-Red filter and the calculated period of the brightness variations displayed by the object in units of days.

The RA and DEC positions for objects in the 2000 Galactic Bulge fields were calculated using a C program designed by Jovan Skuljan of the University of Canterbury for the MOA project. This program uses the Guide Star Catalog (GSC) to transform CCD pixel coordinates to RA and DEC coordinates and requires the $\mathrm{x}$ and $\mathrm{y}$ position of the objects on the CCD and the standard MOA field name. The $\mathrm{x}$ and $\mathrm{y}$ position for each object was determined during the reduction process and recorded in the lightcurve file by the extraction program.

RA and DEC coordinates for objects located in the 1997 LMC fields were determined with images from the ESO (European Southern Observatory) online digitised sky survey (http://archive.eso.org/dss/dss). These digital images, which contained the RA and DEC coordinates, were compared to the 1997 MOA LMC reference fields, which contained the CCD pixel coordinates of the Cepheid variables and eclipsing binaries. The mean brightness and period values were calculated by functions in the extraction program and 
recorded in the lightcurve file ${ }^{1}$.

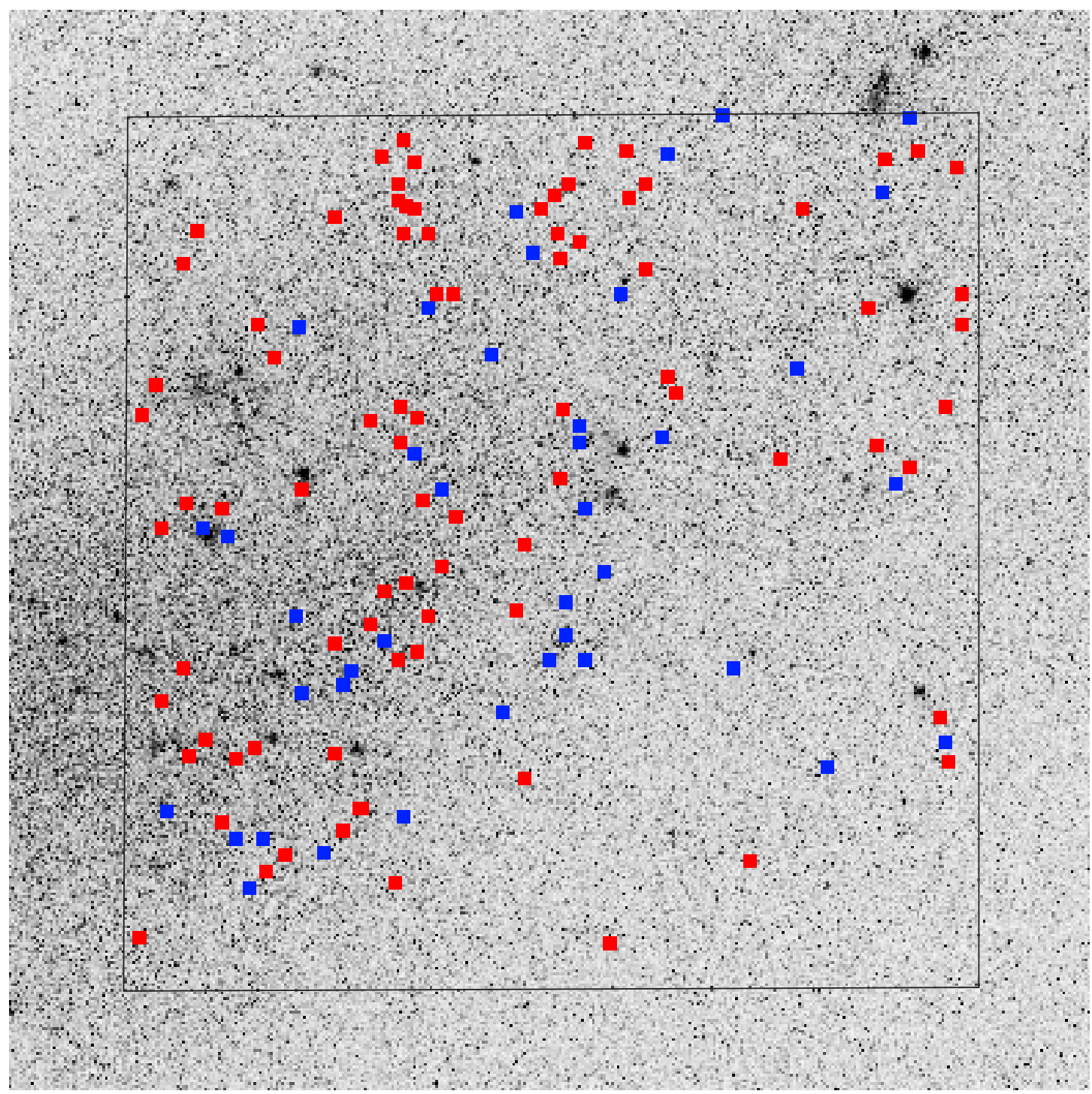

Figure C.1: The figure shows the position of variable stars found in the MOA nlmc 1 field. The Cepheid variables are represented in red and the eclipsing binaries are represented in blue. The position of the MOA nlmc 1 has been overlaid an image of the LMC from the ESO online digitized sky survey. As expected, the distribution of variable stars appears correlated with the stellar density of the field.

\footnotetext{
${ }^{1}$ An example of the lightcurve file containing the $\mathrm{x}$ and $\mathrm{y}$ pixel positions, the mean brightness and the period of pulsation is shown in appendix A.2.2.
} 


\section{C.1 Catalogue of MOA LMC Cepheid variables}

\begin{tabular}{|c|c|c|c|c|c|}
\hline Name & Field & RA $h^{\circ}{ }^{\circ} m^{\prime} \mathbf{s s}^{\prime \prime}$ & Dec $\mathrm{dd}^{\circ} \mathbf{m m}^{\prime} \mathrm{ss}^{\prime \prime}$ & Mean & Period \\
\hline cepheid12369 & "nlmc 3-3 & 050828.77 & -690749.34 & $\overline{15.02}$ & 2.788 \\
\hline cepheid12321 & nlmc 3-3 & 050829.26 & -690851.42 & 14.86 & 3.380 \\
\hline cepheid8699 & nlmc $0-3$ & 050846.84 & -685945.84 & 12.92 & 13.904 \\
\hline cepheid7475 & $\operatorname{lmc} 0-9$ & 050847.50 & -693933.91 & 14.86 & 3.586 \\
\hline cepheid9644 & nlmc $3-3$ & 050851.92 & -691447.48 & 14.78 & 3.586 \\
\hline 47140 & nlmc $0-9$ & 050854.22 & -693640.02 & 13.18 & 0.904 \\
\hline cepheid5611 & nlmc $0-6$ & 050924.88 & -691929.10 & 14.14 & 3.781 \\
\hline cepheid5416 & nlmc $0-3$ & 050926.86 & -685802.74 & 14.44 & 4.455 \\
\hline cepheid3688 & nlmc $0-6$ & 050949.28 & -691730.35 & 15.07 & 2.758 \\
\hline 13082 & $0-3$ & 953.91 & -685930.71 & 13.36 & 8.190 \\
\hline cepheid3045 & nlmc $3-3$ & 050959.54 & -690851.25 & 14.10 & 5.792 \\
\hline 8250 & $1-3$ & 1057.24 & -690140.96 & 12.88 & 15.129 \\
\hline cepheid7268 & $n \operatorname{lmc} 1-6$ & 051110.36 & -691842.30 & 14.55 & 2.483 \\
\hline 0687 & nlmc $2-9$ & 051133.63 & -694751.85 & 14.86 & 3.505 \\
\hline cepheid17268 & nlmc 3-2 & 051229.34 & -691402.90 & 13.28 & 10.348 \\
\hline cepheid15921 & nlmc 3-2 & 051237.58 & -691239.43 & 14.50 & 3.993 \\
\hline cepheid6800 & nlmc $0-2$ & 051247.14 & -690019.53 & 13.20 & 4.988 \\
\hline cepheid13743 & nlmc 3-2 & 051250.33 & -690625.06 & 13.66 & 6.881 \\
\hline cepheid6254 & nlmc $0-2$ & 051253.61 & -685722.52 & 14.71 & 3.486 \\
\hline cepheid6142 & nlmc $0-2$ & 051254.17 & -690137.58 & 15.49 & 1.929 \\
\hline cepheid4604 & nlmc 3-8 & 051318.09 & -695256.47 & 14.88 & 2.706 \\
\hline cepheid3032 & nlmc $0-2$ & 05133205 & -685638.80 & 14.83 & 2.779 \\
\hline cepheid1663 & nlmc $0-2$ & 051347.35 & -690432.60 & 14.70 & 3.359 \\
\hline cepheid3041 & nlmc 3-2 & 051354.40 & -690513.10 & 14.74 & 3.362 \\
\hline cepheid1080 & nlmc $0-2$ & 051354.53 & -690030.82 & 15.00 & 2.916 \\
\hline cepheid13171 & nlmc $1-5$ & 051400.58 & -691559.97 & 13.86 & 2.199 \\
\hline cepheid10966 & nlmc $1-2$ & 051403.55 & -690354.86 & 14.01 & 4.909 \\
\hline cepheid12593 & nlmc $1-5$ & 051404.07 & -692119.68 & 13.55 & 43.662 \\
\hline cepheid10835 & nlmc 1-2 & 051404.61 & -690042.64 & 15.02 & 2.907 \\
\hline cepheid10134 & nlmc $1-2$ & 051412.21 & -690122.63 & 14.24 & 5.151 \\
\hline cepheid10021 & nlmc $1-5$ & 051427.27 & -692512.67 & 13.70 & 4.458 \\
\hline cepheid9436 & nlmc $1-8$ & 051427.93 & -694229.63 & 14.84 & 3.417 \\
\hline cepheid9157 & nlmc $2-5$ & 051441.96 & -693036.94 & 15.01 & 2.553 \\
\hline
\end{tabular}


Table C.1 continued

\begin{tabular}{|c|c|c|c|c|c|}
\hline Name & Field & RA hh ${ }^{\circ} \mathbf{m m}^{\prime} \mathrm{ss}^{\prime \prime}$ & Dec $\mathrm{dd}^{\circ} \mathbf{m m}^{\prime} \mathrm{ss}^{\prime \prime}$ & Mean & Period \\
\hline cepheid4991 & "nlmc $1-5$ & 051513.28 & -692301.21 & 13.54 & $\overline{77.877}$ \\
\hline cepheid4708 & nlmc $2-2$ & 051517.42 & -690751.19 & 14.62 & 3.446 \\
\hline cepheid4548 & nlmc $2-5$ & 051523.09 & -692632.88 & 14.42 & 3.919 \\
\hline cepheid1618 & nlmc $1-2$ & 051536.90 & -690354.08 & 13.28 & 11.089 \\
\hline cepheid2291 & nlmc 2-2 & 051537.78 & -690750.40 & 14.74 & 3.540 \\
\hline cepheid2247 & nlmc $2-2$ & 051538.63 & -691500.60 & 13.64 & 7.286 \\
\hline cepheid8169 & nlmc $0-4$ & $05 \quad 1543.32$ & -692201.11 & 14.40 & 4.285 \\
\hline cepheid1457 & nlmc 2-2 & 051546.16 & -691413.18 & 14.64 & 3.446 \\
\hline cepheid10409 & nlmc 3-4 & 051547.30 & -693033.90 & 14.68 & 3.241 \\
\hline cepheid10077 & nlmc $0-1$ & 051549.61 & -690222.83 & 15.20 & 2.294 \\
\hline cepheid1170 & nlmc $2-5$ & 051552.31 & -693037.77 & 14.62 & 3.241 \\
\hline cepheid9207 & nlmc $0-1$ & 051553.28 & -690142.54 & 14.42 & 2.780 \\
\hline cepheid7454 & nlmc $0-4$ & 051554.53 & -692148.23 & 14.71 & 4.152 \\
\hline cepheid8691 & nlmc $0-1$ & 051555.97 & -685739.09 & 14.81 & 3.026 \\
\hline cepheid8479 & nlmc $0-1$ & 051557.81 & -685636.00 & 14.99 & 2.896 \\
\hline cepheid8706 & nlmc $0-1$ & 051557.85 & -690359.03 & 14.21 & 4.806 \\
\hline cepheid9577 & nlmc $3-4$ & 051558.03 & -693220.95 & 13.43 & 8.675 \\
\hline cepheid9275 & nlmc 3-4 & 051600.01 & -692833.72 & 14.67 & 1.664 \\
\hline cepheid8199 & nlmc $0-1$ & 051601.53 & -685924.02 & 14.10 & 5.686 \\
\hline cepheid10601 & nlmc $3-7$ & 051601.66 & -694732.21 & 14.85 & 3.196 \\
\hline cepheid8994 & nlmc 3-4 & 05160437 & -692833.32 & 13.34 & 6.169 \\
\hline cepheid7964 & nlmc $0-1$ & 051605.40 & -690121.00 & 14.71 & 3.485 \\
\hline cepheid8745 & nlmc $3-4$ & 051607.84 & -693243.86 & 13.27 & 10.242 \\
\hline cepheid10336 & nlmc 3-1 & 051608.39 & -691459.62 & 14.14 & 6.777 \\
\hline cepheid7300 & nlmc $0-1$ & 051613.10 & -685717.06 & 14.81 & 3.087 \\
\hline cepheid7503 & nlmc 3-4 & 051620.77 & -693018.90 & 15.00 & 60.904 \\
\hline cepheid7487 & nlmc $0-7$ & 051636.27 & -694238.88 & 14.54 & 2.379 \\
\hline cepheid6395 & nlmc 3-7 & 051648.14 & -694423.08 & 13.90 & 41.460 \\
\hline cepheid4318 & nlmc $0-1$ & 051651.97 & -690125.53 & 13.60 & 9.201 \\
\hline cepheid5938 & nlmc $0-7$ & 051652.43 & -693914.77 & 14.56 & 3.976 \\
\hline cepheid4342 & nlmc 3-4 & 051659.38 & -693038.64 & 14.67 & 3.568 \\
\hline cepheid1731 & nlmc $0-4$ & 051723.11 & -692101.45 & 13.44 & 8.165 \\
\hline cepheid2308 & nlmc 3-1 & 051733.83 & -691206.03 & 14.80 & 2.979 \\
\hline cepheid12213 & nlmc $2-7$ & 051738.14 & -694528.30 & 14.44 & 5.571 \\
\hline
\end{tabular}


Table C.1 continued

\begin{tabular}{|l|l|r|r|r|r|}
\hline Name & Field & RA hh $^{\circ} \mathbf{m m}^{\prime} \mathbf{s s}^{\prime \prime}$ & Dec dd $^{\circ} \mathbf{m m}^{\prime} \mathbf{s s}^{\prime \prime}$ & Mean & Period \\
\hline \hline cepheid1623 & nlmc 3-1 & 051739.02 & -690930.58 & 14.68 & 3.079 \\
\hline cepheid10522 & nlmc 2-7 & 05175807 & -694726.52 & 14.74 & 3.045 \\
\hline cepheid10723 & nlmc 1-7 & 051759.79 & -693901.08 & 14.18 & 4.815 \\
\hline cepheid9642 & nlmc 1-7 & 051809.94 & -693909.92 & 14.54 & 3.788 \\
\hline cepheid8550 & nlmc 1-7 & 051820.43 & -694327.03 & 14.90 & 15.847 \\
\hline cepheid5772 & nlmc 1-4 & 051824.48 & -692154.55 & 12.93 & 13.522 \\
\hline cepheid5841 & nlmc 1-7 & 051844.93 & -693829.19 & 13.75 & 6.456 \\
\hline cepheid4178 & nlmc 1-1 & 051846.77 & -690317.85 & 13.66 & 71.191 \\
\hline cepheid3594 & nlmc 1-4 & 051851.87 & -692133.47 & 14.64 & 2.961 \\
\hline cepheid4863 & nlmc 1-7 & 051854.35 & -693920.51 & 14.19 & 4.206 \\
\hline cepheid4228 & nlmc 1-7 & 051859.42 & -693411.81 & 14.58 & 3.721 \\
\hline cepheid2668 & nlmc 2-1 & 051902.17 & -690636.18 & 15.28 & 2.939 \\
\hline cepheid2582 & nlmc 2-1 & 051905.04 & -691307.86 & 14.47 & 1.931 \\
\hline cepheid2191 & nlmc 1-4 & 051910.72 & -692259.82 & 14.47 & 3.497 \\
\hline cepheid2381 & nlmc 1-7 & 051917.11 & -693625.12 & 12.75 & 13.284 \\
\hline cepheid1359 & nlmc 2-1 & 051919.09 & -691454.46 & 14.02 & 5.568 \\
\hline cepheid1585 & nlmc 2-7 & 051927.74 & -695159.11 & 14.43 & 17.500 \\
\hline
\end{tabular}

Table C.1: The table shows the properties of the 84 Cepheid variables found in the MOA LMC fields. 


\section{C.2 Catalogue of MOA LMC eclipsing binaries}

\begin{tabular}{|c|c|c|c|c|c|}
\hline Name & Field & RA hh ${ }^{\circ} \mathbf{m m}^{\prime} \mathbf{s s}^{\prime \prime}$ & Dec $\mathrm{dd}^{\circ} \mathbf{m m}^{\prime} \mathrm{ss}^{\prime \prime}$ & Mean & Period \\
\hline eclipsing7293 & "nlmc $0-9$ & 050850.38 & -693806.09 & 16.11 & 1.174 \\
\hline eclipsing5508 & nlmc $0-3$ & 05092670 & -685453.99 & 14.26 & 1.339 \\
\hline eclipsing4436 & nlmc $0-6$ & 050939.70 & -692038.69 & 13.76 & 1.990 \\
\hline eclipsing3460 & nlmc $0-3$ & 050948.81 & -690157.59 & 16.02 & 0.688 \\
\hline eclipsing693 & nlmc $0-9$ & 051017.56 & -693953.92 & 16.07 & 1.032 \\
\hline eclipsing8564 & nlmc $2-3$ & 051100.48 & -691320.34 & 15.21 & 1.076 \\
\hline eclipsing5297 & nlmc $2-6$ & 051122.46 & -693333.88 & 16.85 & 1.361 \\
\hline eclipsing3924 & nlmc $1-3$ & 051139.34 & -685455.85 & 12.82 & 2.556 \\
\hline eclipsing9411 & nlmc $0-2$ & 051218.20 & -685815.88 & 16.31 & 2.390 \\
\hline eclipsing 8100 & nlmc $0-5$ & 051235.97 & -691758.89 & 15.74 & 1.150 \\
\hline eclipsing10089 & nlmc 3-2 & 051312.07 & -690809.90 & 16.02 & 1.240 \\
\hline eclipsing5078 & nlmc $3-5$ & 051319.57 & -692727.91 & 16.42 & 1.788 \\
\hline eclipsing3193 & nlmc $0-5$ & 051336.04 & -692258.94 & 15.66 & 1.054 \\
\hline eclipsing3166 & nlmc $3-5$ & 051338.98 & -693247.94 & 14.89 & 2.729 \\
\hline eclipsing2674 & nlmc $0-5$ & 051341.98 & -691856.29 & 12.76 & 1.789 \\
\hline eclipsing2536 & nlmc $0-5$ & 051344.10 & -691710.88 & 16.11 & 0.777 \\
\hline eclipsing1738 & nlmc $3-5$ & 05134828 & -692941.86 & 13.54 & 3.219 \\
\hline eclipsing2301 & nlmc $3-5$ & 051351.97 & -693159.63 & 15.86 & 1.531 \\
\hline eclipsing13723 & nlmc $2-5$ & 051358.81 & -693208.92 & 13.45 & 1.609 \\
\hline eclipsing9262 & nlmc $1-2$ & 051422.69 & -690509.55 & 16.31 & 1.640 \\
\hline eclipsing8324 & nlmc $1-2$ & 051432.18 & -690133.38 & 17.80 & 3.192 \\
\hline eclipsing8066 & nlmc $1-8$ & 051441.50 & -693801.82 & 16.90 & 2.165 \\
\hline eclipsing6708 & nlmc $2-2$ & 051459.94 & -691106.35 & 17.49 & 0.728 \\
\hline eclipsing5462 & nlmc $0-4$ & 051526.10 & -692032.93 & 14.47 & 3.634 \\
\hline eclipsing2378 & nlmc 2-2 & 051536.69 & -690758.99 & 15.53 & 3.524 \\
\hline eclipsing1616 & nlmc $1-5$ & 051544.35 & -691730.30 & 15.48 & 4.566 \\
\hline eclipsing11441 & nlmc $0-7$ & 051554.09 & -694235.26 & 13.90 & 15.810 \\
\hline eclipsing8138 & nlmc 3-4 & 051614.80 & -693133.89 & 14.94 & 3.213 \\
\hline eclipsing5764 & nlmc 3-4 & 051642.63 & -693242.50 & 14.96 & 2.800 \\
\hline eclipsing5746 & nlmc 3-4 & 051642.93 & -693357.31 & 15.49 & 5.088 \\
\hline eclipsing2138 & nlmc 2-1 & 051655.53 & -690924.40 & 15.84 & 1.136 \\
\hline eclipsing 4837 & nlmc 3-7 & 051705.48 & -694533.46 & 15.20 & 1.432 \\
\hline eclipsing2654 & nlmc $3-4$ & 051719.31 & -693400.01 & 15.70 & 4.720 \\
\hline
\end{tabular}


Table C.2 continued

\begin{tabular}{|l|l|r|r|r|r|}
\hline Name & Field & RA hh $^{\circ} \mathbf{m m}^{\prime} \mathbf{s s}^{\prime \prime}$ & Dec dd $^{\circ} \mathbf{m m}^{\prime} \mathbf{S s}^{\prime \prime}$ & Mean & Period \\
\hline \hline eclipsing1820 & nlmc 3-4 & 051728.73 & -692806.78 & 15.20 & 2.211 \\
\hline eclipsing1684 & nlmc 3-7 & 051740.51 & -694526.73 & 13.59 & 3.711 \\
\hline eclipsing9732 & nlmc 2-7 & 051806.00 & -694825.42 & 14.01 & 1.553 \\
\hline eclipsing9755 & nlmc 1-7 & 051809.48 & -694448.92 & 15.12 & 2.669 \\
\hline eclipsing5530 & nlmc 1-4 & 051828.11 & -692344.54 & 16.74 & 2.184 \\
\hline eclipsing4811 & nlmc 1-4 & 051836.62 & -692343.66 & 16.01 & 2.765 \\
\hline eclipsing2819 & nlmc 1-7 & 051915.15 & -694231.63 & 14.61 & 2.726 \\
\hline
\end{tabular}

Table C.2: The table shows the properties of the four eclipsing binaries found in the first search of the MOA LMC fields and the properties of the 40 eclipsing binaries found in the second search of the same fields. 


\section{C.3 Catalogue of MOA Galactic Bulge eclipsing binaries}

\begin{tabular}{|l|l|r|r|r|r|}
\hline Name & Field & RA $_{\text {hh }}{ }^{\circ} \mathbf{m m}^{\prime} \mathbf{s s}^{\prime \prime}$ & Dec dd $^{\circ} \mathbf{m m}^{\prime} \mathbf{s s}^{\prime \prime}$ & Mean & Period \\
\hline \hline eclipsing486 & ngb 1-3 & 175436.29 & -290347.70 & 11.67 & 2.536 \\
\hline eclipsing25986 & ngb 1-3 & 175457.21 & -294141.52 & 11.13 & 7.424 \\
\hline eclipsing13327 & ngb 1-3 & 175503.05 & -295303.54 & 11.01 & 1.678 \\
\hline eclipsing9581 & ngb 1-2 & 175523.89 & -291243.66 & 11.38 & 3.582 \\
\hline eclipsing46 & ngb 1-1 & 175528.48 & -293406.93 & 12.20 & 2.549 \\
\hline eclipsing4631 & ngb 1-1 & 175533.75 & -294624.89 & 12.56 & 5.355 \\
\hline eclipsing16391 & ngb 1-3 & 175559.46 & -295027.61 & 11.32 & 4.534 \\
\hline eclipsing34328 & ngb 1-2 & 175559.54 & -293034.07 & 11.45 & 2.161 \\
\hline eclipsing2476 & ngb 1-1 & 175601.45 & -294032.77 & 12.65 & 1.352 \\
\hline eclipsing26988 & ngb 1-3 & 175745.93 & -284032.45 & 11.21 & 1.658 \\
\hline eclipsing1488 & ngb 1-1 & 175748.67 & -293738.51 & 11.94 & 6.318 \\
\hline eclipsing5174 & ngb 1-2 & 175800.88 & -290915.64 & 11.04 & 1.280 \\
\hline eclipsing23546 & ngb 1-2 & 175823.82 & -295314.67 & 11.78 & 3.647 \\
\hline eclipsing1777 & ngb 1-1 & 175825.55 & -293822.57 & 12.51 & 2.298 \\
\hline eclipsing2143 & ngb 2-2 & 175920.64 & -294522.21 & 12.08 & 12.156 \\
\hline eclipsing5441 & ngb 2-3 & 175955.26 & -291357.89 & 12.03 & 0.639 \\
\hline eclipsing784 & ngb 2-2 & 180126.08 & -293932.28 & 12.19 & 3.720 \\
\hline eclipsing1010 & ngb 2-2 & 180154.84 & -294028.14 & 11.48 & 1.594 \\
\hline eclipsing5286 & ngb 2-2 & 180204.03 & -295919.77 & 12.08 & 2.139 \\
\hline
\end{tabular}

Table C.3: The table shows the properties of the nineteen eclipsing binaries found in the MOA ngb 1 and ngb 2 fields. 


\section{C.4 Catalogue of MOA Galactic Bulge potential planetary} transits

\begin{tabular}{|l|l|r|r|r|r|}
\hline Name & Field & RA hh $\mathbf{~ m m}^{\prime}$ ss $^{\prime \prime}$ & Dec $\mathbf{d d}^{\circ} \mathbf{m m}^{\prime} \mathbf{s s}^{\prime \prime}$ & Mean & Period \\
\hline \hline \multicolumn{7}{|c|}{ Very interesting category } \\
\hline transit5829 & ngb 2-2 & 180249.01 & -300158.03 & 11.89 & 7.480 \\
\hline \hline \multicolumn{7}{|c|}{ Interesting categorie } \\
\hline transit12414 & ngb 1-2 & 175705.74 & -291454.07 & 11.65 & 3.225 \\
\hline transit18269 & ngb 1-3 & 175754.92 & -284841.94 & 10.99 & 5.617 \\
\hline transit2722 & ngb 1-1 & 175813.91 & -294100.66 & 12.19 & 4.082 \\
\hline transit228 & ngb 2-2 & 175905.78 & -293652.76 & 11.71 & 2.036 \\
\hline transit5610 & ngb 2-2 & 175921.46 & -300104.41 & 11.74 & 6.581 \\
\hline transit5479 & ngb 2-3 & 175935.48 & -291351.78 & 11.63 & 4.722 \\
\hline transit4741 & ngb 2-1 & 180023.07 & -302738.18 & 11.81 & 2.512 \\
\hline transit3816 & ngb 2-1 & 180048.61 & -302241.84 & 12.50 & 7.314 \\
\hline transit3175 & ngb 2-1 & 180119.99 & -301930.27 & 12.11 & 4.298 \\
\hline transit1337 & ngb 2-1 & 180127.21 & -301024.40 & 12.23 & 2.088 \\
\hline transit2859 & ngb 2-1 & 180133.85 & -301744.15 & 12.06 & 4.881 \\
\hline transit2949 & ngb 2-3 & 180155.51 & -292231.55 & 11.84 & 2.949 \\
\hline transit3156 & ngb 2-2 & 180247.14 & -294915.00 & 11.98 & 4.082 \\
\hline transit4541 & ngb 2-2 & 180247.15 & -295552.86 & 11.99 & 2.685 \\
\hline transit335 & ngb 2-1 & 180253.91 & -300511.20 & 11.84 & 3.859 \\
\hline
\end{tabular}

Table C.4: The table shows the properties of the fifteen interesting and one very interesting potential planetary transits found in the MOA ngb 1 and ngb 2 fields. 


\section{Bibliography}

Alard, C. \& Lupton, R. H. (1998), Astrophysical Journal 503, 325+.

Alcaino, G., Liller, W. \& Alvarado, F. (1989), Astronomy and Astrophysics 216, 68-79.

Alcock, C., Allen, W. H., Allsman, R. A., Alves, D., Axelrod, T. S., Banks, T. S., Beaulieu, S. F., Becker, A. C., Becker, R. H., Bennett, D. P., Bond, I. A., Carter, B. S., Cook, K. H., Dodd, R. J., Freeman, K. C., Gregg, M. D., Griest, K., Hearnshaw, J. B., Heller, A., Honda, M., Jugaku, J., Kabe, S., Kaspi, S., Kilmartin, P. M., Kitamura, A., Kovo, O., Lehner, M. J., Love, T. E., Maoz, D., Marshall, S. L., Matsubara, Y., Minniti, D., Miyamoto, M., Morse, J. A., Muraki, Y., Nakamura, T., Peterson, B. A., Phillips, M. M., Pratt, M. R., Quinn, P. J., Reid, I. N., Reid, M., Reiss, D., Retter, A., Rodgers, A. W., Sargent, W. L. W., Sato, H., Sekiguchi, M., Stetson, P. B., Stubbs, C. W., Sullivan, D. J., Sutherland, W., Tomaney, A., Vandehei, T., Watase, Y., Welch, D. L., Yanagisawa, T., Yoshizawa, M., Yock, P. C. M., The Macho \& Gman Collaborations (1997), Astrophysical Journal 491, 436-450.

Alcock, C., Allsman, R. A., Alves, D., Ansari, R., Aubourg, E., Axelrod, T. S., Bareyre, P., Beaulieu, J.-P., Becker, A. C., Bennett, D. P., Brehin, S., Cavalier, F., Char, S., Cook, K. H., Ferlet, R., Fernandez, J., Freeman, K. C., Griest, K., Grison, P., Gros, M., Gry, C., Guibert, J., Lachieze-Rey, M., Laurent, B., Lehner, M. J., Lesquoy, E., Magneville, C., Marshall, S. L., Maurice, E., Milsztajn, A., Minniti, D., Moniez, M., Moreau, O., Moscoso, L., Palanque-Delabrouille, N., Peterson, B. A., Pratt, M. R., Prevot, L., Queinnec, F., Quinn, P. J., Renault, C., Rich, J., Spiro, M., Stubbs, C. W., Sutherland, W., Tomaney, A., Vandehei, T., Vidal-Madjar, A., Vigroux, L. \& Zylberajch, S. (1998), Astrophysical Journal Letters 499, L9-L12.

Alcock, C., Allsman, R. A., Alves, D., Axelrod, T. S., Bennett, D. P., Cook, K. H., Freeman, K. C., Griest, K., Guern, J., Lehner, M. J., Marshall, S. L., Peterson, B. A., Pratt, M. R., Quinn, P. J., Rodgers, A. W., Stubbs, C. W. \& Sutherland, W. (1995), Astrophysical Journal Letters 454, L125-L128. 
Alcock, C., Allsman, R. A., Alves, D. R., Axelrod, T. S., Becker, A. C., Bennett, D. P., Cook, K. H., Dalal, N., Drake, A. J., Freeman, K. C., Geha, M., Griest, K., Lehner, M. J., Marshall, S. L., Minniti, D., Nelson, C. A., Peterson, B. A., Popowski, P., Pratt, M. R., Quinn, P. J., Stubbs, C. W., Sutherland, W., Tomaney, A. B., Vandehei, T. \& Welch, D. L. (2001), Astrophysical Journal Letters 550, L169-L172.

Alcock, C., Allsman, R. A., Alves, D. R., Axelrod, T. S., Becker, A. C., Bennett, D. P., Cook, K. H., Dalal, N., Drake, A. J., Freeman, K. C., Geha, M., Griest, K., Lehner, M. J., Marshall, S. L., Minniti, D., Nelson, C. A., Peterson, B. A., Popowski, P., Pratt, M. R., Quinn, P. J., Stubbs, C. W., Sutherland, W., Tomaney, A. B., Vandehei, T. \& Welch, D. (2000), Astrophysical Journal 542, 281-307.

Barnothy, J. M. (1965), Astrophysical Journal 70, 666-698.

Bayne, G., Tobin, W., Pritchard, J. D., Bond, I., Pollard, K. R., Besier, S. C., Noda, S., Sumi, T., Yanagisawa, T., Sekiguchi, M., Honda, M., Muraki, Y., Takeuti, M., Hearnshaw, J. B., Kilmartin, P. M., Dodd, R. J., Sullivan, D. J. \& Yock, P. C. M. (2002), MNRAS 331, 609-614.

Bevington, P. R. (1969), Data Reduction and Error Analysis for the Physical Science, 1 edn, McGraw-Hill Book Company.

Bond, I. A., Abe, F., Dodd, R. J., Hearnshaw, J. B., Honda, M., Jugaku, J., Kilmartin, P. M., Marles, A., Masuda, K., Matsubara, Y., Muraki, Y., Nakamura, T., Nankivell, G., Noda, S., Noguchi, C., Ohnishi, K., Rattenbury, N. J., Reid, M., Saito, T., Sato, H., Sekiguchi, M., Skuljan, J., Sullivan, D. J., Sumi, T., Takeuti, M., Watase, Y., Wilkinson, S., Yamada, R., Yanagisawa, T. \& Yock, P. C. M. (2001), MNRAS 327, 868-882.

Bond, I. A., Abe, F., Dodd, R. J., Hearnshaw, J. B., Kilmartin, P. M., Masuda, K., Matsubara, Y., Muraki, Y., Noda, S., Petterson, O. K. L., Rattenbury, N. J., Reid, M., Saito, T., Saito, Y., Sako, T., Skuljan, J., Sullivan, D. J., Sumi, T., Wilkinson, S., Yamada, R., Yanagisawa, T. \& Yock, P. C. M. (2002), MNRAS 331, L19-L23.

Bond, I. A., Rattenbury, N. J., Skuljan, J., Abe, F., Dodd, R. J., Hearnshaw, J. B., Honda, M., Jugaku, J., Kilmartin, P. M., Marles, A., Masuda, K., Matsubara, Y., Muraki, Y., Nakamura, T., Nankivell, G., Noda, S., Noguchi, C., Ohnishi, K., Reid, M., Saito, T., Sato, H., Sekiguchi, M., Sullivan, D. J., Sumi, T., Takeuti, M., Watase, Y., Wilkinson, S., Yamada, R., Yanagisawa, T. \& Yock, P. C. M. (2002), MNRAS 333, 71-83. 
Brown, T. \& Charbonneau, D. (1999), The stare project; a transit search for hot jupiters, in 'American Astronomical Society Meeting', Vol. 195, A.S.P. Conference Series, pp. 1534-1539.

Brown, T., Charbonneau, D., Gilliland, R. L., Noyes, R. W. \& Burrows, A. (2001), Astrophysical Journal 552, 699-709.

Castellano, T., Jenkins, J., Trilling, D. E., Doyle, L. \& Koch, D. (2000), Astrophysical Journal Letters 532, L51-L53.

Charbonneau, D., Brown, T. M., Latham, D. W. \& Mayor, M. (2000), Astrophysical Journal Letters 529, L45-L48.

Chwolson, O. (1924), Astron. Nachr. 221, 329.

Eddington, A. S. (1917), The Observatory 40, 290-293.

Einstein, A. (1911), Ann. Phys. 35, 898.

Einstein, A. (1916), Ann. Phys. 49, 769.

Gilliland, R., Brown, T. M., Guhathakurta, P., Sarajedini, A., Milone, E. F., Albrow, M. D., Baliber, N. R., Bruntt, H., Burrows, A., Charbonneau, D., Choi, P., Cochran, W. D., Edmonds, P. D., Frandsen, S., Howell, J. H., Lin, D. N. C., Marcy, G. W., Mayor, M., Naef, D., Sigurdsson, S., Stagg, C. R., Vandenberg, D. A., Vogt, S. S. \& Williams, M. D. (2000), Astrophysical Journal Letters 545, L47-L51.

Glass, I. S. \& Evans, T. L. (1981), Nature 291, 303-304.

Griest, K. (1991), Astrophysical Journal 366, 412-421.

Griest, K. \& Safizadeh, N. (1998), Astrophysical Journal 500, 37-50.

Groenewegen, M. \& Oudmaijer, R. (2000), Astronomy and Astrophysics 356, 849-872.

Harris, W. (1996), Astrophysical Journal 112, 1487-1488.

Hearnshaw, J. B., Bond, I. A., Rattenbury, N. J., Noda, S., Takeuti, M., Abe, F., Carter, B. S., Dodd, R. J., Honda, M., Jugaku, J., Kabe, S., Kilmartin, P. M., Koribalski, B. S., Matsubara, Y., Masuda, K., Muraki, Y., Nakamura, T., Nankivell, G. R., Reid, M., Rumsey, N. J., Saito, T., Sato, H., Sekiguchi, M., Sullivan, D. J., Sumi, T., Watase, Y., Yanagisawa, T., Yock, P. C. M. \& Yoshizawa, M. (2000), Photometry of pulsating stars in the magellanic clouds as observed in the moa project, in 'ASP Conf. Ser. 203: IAU Colloq. 176: The Impact of Large-Scale Surveys on Pulsating Star Research', pp. 31-37. 
Henry, G. W., Marcy, G. W., Butler, R. P. \& Vogt, S. S. (2000), Astrophysical Journal Letters 529, L41-L44.

Jha, S., Charbonneau, D., Garnavich, P. M., Sullivan, D. J., Sullivan, T., Brown, T. M. \& Tonry, J. L. (2000), Astrophysical Journal Letters 540, L45-L51.

Lane, B. F., Kuchner, M. J., Boden, A. F., Creech-Eakman, M. \& Kulkarni, S. R. (2000), Nature 407, 485-487.

Lanoix, P., Paturel, G. \& Garnier, R. (1999), MNRAS 308, 969-978.

Lasserre, T., Afonso, C., Albert, J. N., Andersen, J., Ansari, R., Aubourg, É., Bareyre, P., Bauer, F., Beaulieu, J. P., Blanc, G., Bouquet, A., Char, S., Charlot, X., Couchot, F., Coutures, C., Derue, F., Ferlet, R., Glicenstein, J. F., Goldman, B., Gould, A., Graff, D., Gros, M., Haıssinski, J., Hamilton, J. C., Hardin, D., de Kat, J., Kim, A., Lesquoy, É., Loup, C., Magneville, C., Mansoux, B., Marquette, J. B., Maurice, É., Milsztajn, A., Moniez, M., Palanque-Delabrouille, N., Perdereau, O., Prévot, L., Regnault, N., Rich, J., Spiro, M., Vidal-Madjar, A., Vigroux, L., Zylberajch, S. \& The EROS collaboration (2000), Astronomy and Astrophysics 355, L39-L42.

Mao, S. \& Paczynski, B. (1991), Astrophysical Journal Letters 374, L37-L40.

Mazeh, T., Naef, D., Torres, G., Latham, D. W., Mayor, M., Beuzit, J., Brown, T. M., Buchhave, L., Burnet, M., Carney, B. W., Charbonneau, D., Drukier, G. A., Laird, J. B., Pepe, F., Perrier, C., Queloz, D., Santos, N. C., Sivan, J., Udry, S. . \& Zucker, S. (2000), Astrophysical Journal Letters 532, L55-L58.

Noda, S. (1999), Study of Variable Stars and Gravitational Microlensing by Photometry of the LMC, MSc thesis, Nagoya University.

Noda, S. (2001), Study of Red Variable Stars in the Large Magellanic Cloud from the MOA Database, MSc thesis, Nagoya University.

Noda, S., Takeuti, M., Abe, F., Bond, I. A., Dodd, R. J., Hearnshaw, J. B., Honda, M., Honma, M., Jugaku, J., Kabe, S., Kan-ya, Y., Kato, Y., Kilmartin, P. M., Matsubara, Y., Masuda, K., Muraki, Y., Nakamura, T., Nankivell, G. R., Noguchi, C., Ohnishi, K., Reid, M., Rattenbury, N. J., Saito, T., Sato, H., Sekiguchi, M., Skuljan, J., Sullivan, D. J., Sumi, T., Watase, Y., Wilkinson, S., Yamada, R., Yanagisawa, T., Yock, P. C. M. \& Yoshizawa, M. (2002), MNRAS 330, 137-152.

Paczynski, B. (1986a), Astrophysical Journal 301, 503-516.

Paczynski, B. (1986b), Astrophysical Journal 304, 1-5. 
Press, W., Flannery, B., Teukolsky, S. \& Vetterling, W. (1988), Numerical recipes in C, 1 edn, Cambridge University Press.

Rattenbury, N. (1999), MJUO Adaptive Optics Feasibility Study and Variable Star Returns from the MOA Database, MSc thesis, University of Auckland.

Refsdal, S. (1964), MNRAS 128, 295-306.

Reid, M. (2002), Observational Gravitational Microlensing, PhD thesis, Victoria University of Wellington.

Rhie, S., Bennett, D. P., Becker, A. C., Peterson, B. A., Fragile, P. C., Johnson, B. R., Quinn, J. L., Crouch, A., Gray, J., King, L., Messenger, B., Thomson, S., Bond, I. A., Abe, F., Carter, B. S., Dodd, R. J., Hearnshaw, J. B., Honda, M., Jugaku, J., Kabe, S., Kilmartin, P. M., Koribalski, B. S., Masuda, K., Matsubara, Y., Muraki, Y., Nakamura, T., Nankivell, G. R., Noda, S., Rattenbury, N. J., Reid, M., Rumsey, N. J., Saito, T., Sato, H., Sato, S., Sekiguchi, M., Sullivan, D. J., Sumi, T., Watase, Y., Yanagisawa, T., Yock, P. C. M. \& Yoshizawa, M. (2000), Astrophysical Journal 533, 378-391.

Schechter, P., Mateo, M. \& Saha, A. (1993), Publications of the Astrononical Soctery of the Pacific 105, 1342-1353.

Schmidt, M. (1963), Nature 197, 1040.

Schwarzenberg-Czerny, A. (1989), MNRAS 241, 153-165.

Schwarzenberg-Czerny, A. (1996), Astrophysical Journal Letters 460, L107-L110.

Shapley, H. (1914), Astrophysical Journal 40, 448-465.

Soldner, J. (1804), Bel. Astron. Jahrb. p. 161.

Sterken, C. \& Jaschek, C. (1996), Light Curves of Variable Stars: a Pictorial Atlas, 1 edn, Cambridge University Press.

Stetson, P. B. (1987), Publications of the Astrononical Soctery of the Pacific 99, 191-222.

Stetson, P. B. (1996), Publications of the Astrononical Soctery of the Pacific 108, 851876.

Udalski, A., Paczynski, B., Zebrun, K., Szymaski, M., Kubiak, M., Soszynski, I., Szewczyk, O., Wyrzykowski, L. \& Pietrzynski, G. (2002), astro-ph/0102184.

Walsh, D., Carswell, R. F. \& Weymann, R. J. (1979), Nature 279, 381. 
Welch, D. L. \& Stetson, P. B. (1993), Astrophysical Journal 105, 1813-1821.

Wells, D., Greisen, E. W. \& Harten, R. H. (1981), Astronomy and Astrophysics 44, 363370.

Weymann, R. J., Chaffee, F. H., Carleton, N. P., Walsh, D., Carswell, R. F. \& Davis, M. (1979), Astrophysical Journal Letters 23, L43.

Witt, H. J. \& Mao, S. (1994), Astrophysical Journal 430, 505-510.

Yanagisawa, T., Muraki, Y., Matsubara, Y., Abe, F., Masuda, K., Noda, S., Sumi, T., Kato, Y., Fujimoto, M., Sato, S., Bond, I., Rattenbury, N., Yock, P., Kilmartin, P., Hearnshaw, J., Reid, M., Sullivan, D., Carter, B., Dodd, R., Nankivell, G., Rumsey, N., Honda, M., Sekiguchi, M., Yoshizawa, M., Nakamura, T., Sato, H., Kabe, S., Kobayashi, M., Watase, Y., Jugaku, J., Saito, T. \& Koribalsky, B. (2000), Experimental Astronomy 10, 519-535.

Zwicky, F. (1937a), Phys. Rev. Letters 51, 290.

Zwicky, F. (1937b), Phys. Rev. Letters 51, 679. 